\title{
Weak Equivalence and the Structures of Cocycles of an Ergodic Automorphism
}

By

\author{
Sergey I. BeZuGlyı* and Valentin Ya. Golodets*
}

\begin{abstract}
Let $(X, \mu)$ be a Lebesgue space, $\Gamma$ an approximately finite ergodic group of the automorphisms, $\alpha$ a cocycle on $X \times \Gamma$ with values in an arbitrary abelian 1.c.s. group $G$, and $\rho$ the Radon-Nikodym cocycle on $X \times \Gamma$. The concept of weak equivalence of the pairs $(\Gamma, \alpha)$ is introduced and studied, which generalizes the concept of trajectory equivalence of automorphism groups. It is proved that the pairs $\left(\Gamma_{1}, \alpha_{0}^{1}\right)$ and $\left(\Gamma_{2}, \alpha_{0}^{2}\right)\left(\alpha_{0}^{i}=(\alpha, \rho)\right)$ are (stably) weakly equivalent iff the corresponding Mackey pairs $W_{1}\left(G_{0}\right)$ and $W_{2}\left(G_{0}\right)$ of the group $G_{0}=$ $\boldsymbol{G} \times \boldsymbol{R}$ are isomorphic. It is proved that any ergodic action of $G \times \mathbb{R}$ (or $G$ ) is isomorphic to the Mackey action associated with a certain pair $\left(\Gamma, \alpha_{0}\right)$. The structure of cocycles of approximately finite equivalence relations is studied. The relationship between the type of the group $\Gamma$ and that of the corresponding Mackey action is considered.
\end{abstract}

\section{§0. Introduction}

The present paper is a study of the countable approximately finite (a.f.) groups $\Gamma$ of automorphisms of measure spaces and the cocycles $\alpha$ for $\Gamma$ taking the values in an abelian locally compact separable (1.c.s.) group $G$. Such cocycles were thoroughly studied in the book by K. Schmidt [15]. He in part considered the important classes of cocycles: transient, lacunary, etc. We shall use the set of all pairs $(\Gamma, \alpha)$ to study an equivalence relation which is called the weak equivalence (see Section 1). In the case where the cocycle $\alpha$ is the Radon-Nikodym cocycle $\rho$, weak equivalence of pairs $\left(\Gamma_{1}, \rho_{1}\right)$ and $\left(\Gamma_{2}, \rho_{2}\right)$ is the same as the well-known orbital equivalence relation of the groups of automorphisms $\Gamma_{1}$ and $\Gamma_{2}$ [9]. The weak equivalence was introduced in [5] (see also [6]) in the study of the pairs $(\Gamma, \alpha)$, where $\Gamma$ is a group of measure-preserving automorphisms and $\alpha$ a cocycle with the dense range in a 1.c.s. amenable group

Communicated by H. Araki, April 16, 1990. Revised August 23, 1990.

1991 Mathematics Subject Classification: 28D15.

* Institute for Low Temperature Physics and Engineering, Ukr. SSR Academy of Sciences, Lenin Ave., 47, Kharkc.y 310164, USSR 
$G$. It was also proved there that all such pairs are weakly equivalent for the fixed $G$. Later the weak equivalence of the pairs $(\Gamma, \alpha)$ was studied in $[1,2,7]$ and elsewhere.

The main purpose of this paper is to describe the structures of cocycles of a.f. groups of automorphisms with values in a 1.c.s. group. It appears that any cocycle is weakly equivalent to a cocycle which in a natural way composed of simpler cocycles: a transient cocycle and a cocycle with a dense range. In solving this problem, we introduced and studied measurable fields of cocycles which have a dense range in an arbitrary subgroup of the group $G_{0}=G \times \mathbb{R}$.

Every pair $\left(\Gamma, \alpha_{0}\right)$, where $\alpha_{0}=(\alpha, \rho)$, defines in a natural way an action of the group $G_{0}$ which is called the Mackey action (or refered to as associated with the pair $\left.\left(\Gamma, \alpha_{0}\right)\right)$ [11]. As a consequence of the above result on the structures of cocycles, we obtain the solution of the problem of finding the necessary and sufficient conditions of weak equivalence of the pairs $\left(\Gamma_{1}, \alpha_{0}^{1}\right)$ and $\left(\Gamma_{2}, \alpha_{0}^{2}\right)$. These conditions consists in the isomorphism of the corresponding Mackey actions of the group $G_{0}$. These studies are based on the methods developed in $[1,5,6]$. Another approach to solution of a similar problem is proposed by A.L. Fedorov [2].

Our results are easy to be extended to the case of the pairs $(\Gamma, \alpha)$, where the cocycle $\alpha$ takes the values in an arbitrary 1.c.s. amenable group $G$ and the Mackey action either is free or has a closed normal subgroup of $G \times \mathbb{R}$ in the capacity of the stabilizer.

The paper is organized as follows. Section 1 presents the information on cocycles, needed for subsequent arguments and taken mainly from [15], and introduced the concept of weak equivalence of the pairs $(\Gamma, \alpha)$. In Section 2 we study the transient cocycles defined for an arbitrary countable group of automorphisms and taking values in a 1.c.s. group $G$. In Section 3, cocycles with a dense range are constructed for an arbitrary closed subgroup $H_{0} \subset G \times \mathbb{R}$ and measurable fields of cocycles with a dense range in $H_{0}$ are studied. It is found that such fields of cocycles are weakly equivalent to a constant field of cocycles. The results of this section are applied to study the cocycles, for which the Mackey action is transitive, in Section 4. Section 5 studies the lacunary cocycles on a.f. groups of measure preserving automorphisms. They correspond to the free Mackey actions of the group $G$, that generally speaking have a quasiinvariant measure. In the subsequent two sections, the general case is considered where the group $\Gamma$ has the quasi-invariant measure and the Mackey action of the group $G_{0}=G \times \mathbb{R}$ is non-free. In the last section we consider 
results on the relation of the types of the Mackey actions and groups $\Gamma$.

\section{§1. Preliminaries. Weak Equivalence}

1.1. In this section we shall provide the preliminary facts from the ergodic theory that we shall need for the subsequent arguments. The definitions and more detailed results can be found in $[8,9,14,15]$.

The set of all non-singular automorphisms of a Lebesgue space $(X, \mathscr{B}, \mu)$ with a continuous measure $\mu$ will be denoted by $\operatorname{Aut}(X, \mathscr{B}, \mu)$. We shall identify automorphisms differing on a measure 0 set. Let $\Gamma$ be a countable subgroup of $\operatorname{Aut}(X, \mathscr{B}, \mu)$. The set $[\Gamma]=\{g \in \operatorname{Aut}(X, \mathscr{B}, \mu): g x \in \Gamma x$ for $\mu$-a.a $x \in X\}$, where $\Gamma x=\{r x: r \in \Gamma\}$ is the orbit of $x$, is called the full group of automorphisms generated by $\Gamma$. The set $N[\Gamma]=\left\{R \in\right.$ Aut $\left.(X, \mathscr{B}, \mu): R[\Gamma] R^{-1}=[\Gamma]\right\}$, which is also a subgroup of $\operatorname{Aut}(X, \mathscr{B}, \mu)$ is called the normalizer of $[\Gamma]$. The group of automorphisms $\Gamma$ is called approximately finite (a.f.), if there exists an automorphism $T \in \operatorname{Aut}(X, \mathscr{B}, \mu)$ such that $[\Gamma]=[T]$, where $[T]=\left[\left\{T^{n}: n \in \mathbb{Z}\right\}\right]$.

The two groups of automorphisms $\Gamma_{1} \subset \operatorname{Aut}\left(X_{1}, \mathscr{B}_{1}, \mu_{1}\right)$ and $\Gamma_{2} \subset \operatorname{Aut}\left(X_{2}\right.$, $\mathcal{B}_{2}, \mu_{2}$ ) are called orbital equivalent, if there exists a one-to-one measurable map $\theta: X_{1} \rightarrow X_{2}$, such that $\theta\left[\Gamma_{1}\right] \theta^{-1}=\left[\Gamma_{2}\right]$ and the measures $\mu_{2}$ and $\theta \circ \mu_{1}$ are equivalent.

The ergodic group $\Gamma \subset \operatorname{Aut}(X, \mathscr{B}, \mu)$ is called a type $\mathrm{II}_{1}\left(\mathrm{II}_{\infty}\right)$ group, if there exists a measure $\nu \sim \mu$, such that $\gamma \circ \nu=\nu$ for all $r \in \Gamma$ and the measure $\nu(X)$ is finite (infinite). If there is no $\Gamma$-invariant measure equivalent to the measure $\mu$, then $\Gamma$ is said to be of type III. Type III may be further classified (see below).

We shall also use sometimes the terminology and facts of the measurable groupoid theory (see, e.g. [3, 12]). The result of this paper may also be fully expressed in terms of this theory. However, as a rule, we use the standard approach to the study of countable groups of automorphisms, since we proceed from the definitions and facts of $[8,9,14,15$, etc.].

1.2. We shall cite the definition of the array as in [10].

Let $\Gamma$ be an ergodic group of automorphisms of $(X, \mathscr{B}, \mu)$. The expression

$$
\xi=(A, \Xi, A(\cdot), r(\cdot, \cdot))
$$

will be called the $\Gamma$-array of the set $A \subset X(\mu A>0)$ provided that the following conditions are fulfilled:

(i) $\Xi$ is a finite set of indices; 
(ii) $\bigcup_{i \in \Xi} A(i)=A, A(i) \cap A(j)=\varnothing(i \neq j), \mu(A(i))>0$;

(iii) $r(i, j)$ are non-singular maps such that $r(i, j) A(j)=A(i), r(i, i)=\mathbb{1}$, $r\left(i_{2}, j\right) r\left(j, i_{1}\right)=r\left(i_{2}, i_{1}\right), r(i, j) x \in \Gamma x$ for a.a. $x \in A(j)$.

Denote by $\mathcal{G}(\xi)$ the finite group of automorphisms of $A$ generated by $\gamma(i, j), i, j \in \Xi$ and $\mathcal{Q}(\xi)$ the collection of sets of the form $\cup_{i \in \Delta} A(i)$, where $\Delta$ is an arbitrary subset in $\Xi$.

The pairs $(A(i), \gamma(j, i)), i, j \in \Xi$ will be called elements of $\xi$.

Let there be defined the two $\Gamma$-arrays: $\xi_{1}=(A, \Xi, A(\cdot), r(\cdot, \cdot))$ and $\xi_{2}=$ $\left(A\left(i_{0}\right), \Omega, B(\cdot), \delta(\circ, \cdot)\right)$, where $i_{0} \in \Xi$. Define a new $\Gamma$-array $\xi_{1} \times \xi_{2}$ which will be called a refinement of $\xi_{1}$ with respect to $\xi_{2}$, according to the equality

$$
\xi_{1} \times \xi_{2}=(A, \Xi \times \Omega, C(\circ, \cdot), \tau(\circ, \cdot ; \cdot, \circ)),
$$

where $C(i, n)=r\left(i, i_{0}\right) B(n), \tau\left(i_{1}, n_{\mathbb{I}} ; i, n\right)=\gamma\left(i_{1}, i_{0}\right) \delta\left(n_{1}, n\right) r\left(i_{0}, i\right), i_{0}, i, i_{\mathbb{1}} \in \Xi, n, n_{\mathbb{1}}$ $\in \Omega$.

If the $\Gamma$-array (1.1) is defined, then it will be said to be defined over the partition $(A, \Xi, A(\cdot))$.

1.3. Let, as earlier, $\Gamma$ be a countable ergodic group of automorphisms of $(X, \mathscr{B}, \mu)$ acting freely and let $G$ be an arbitrary 1.c.s. abelian group.

Definition 1.1. A measurable map $\alpha: X \times \Gamma \rightarrow G$ is called a cocycle, if for any $r_{1}, r_{2} \in \Gamma$ and $\mu-a . a x \in X$

$$
\alpha\left(x, r_{1} r_{2}\right)=\alpha\left(r_{2} x, r_{1}\right) \alpha\left(x, r_{2}\right)
$$

The set of all cocycles will be denoted by $Z^{1}(X \times \Gamma, G)$.

An example of a cocycle is the following cocycle $\rho: X \times \Gamma \rightarrow \mathbb{R}$

$$
\rho(x, \gamma)=\log \frac{d \gamma^{-1} \circ \mu}{d \mu}(x)
$$

which is called the Radon-Nikodym cocycle.

By $\mathscr{R}(\Gamma)$ we shall denote the measurable ergodic equivalence relation on $X$ generated by partition into orbits of the group $\Gamma$. Then, any cocycle $\alpha \in Z^{1}(X \times \Gamma, G)$ defines the map $u_{\alpha}: \mathscr{R}(\Gamma) \rightarrow G$ which is called an orbital cocycle and is defined by the formula

$$
u_{\alpha}(y, x)=\alpha(x, \gamma),
$$

where $y=r x$ and $r \in \Gamma$ is found for $x, y$ uniquely, because $\Gamma$ acts freely. From (1.2) and (1.3) it follows that the orbital cocycle $u: \mathscr{R}(\Gamma) \rightarrow G$ satisfies the re- 
lation $u(z, x)=u(z, y) u(y, x)$, where $(z, x),(z, y),(y, x) \in \mathscr{R}(\Gamma)$. The reverse statement is also true: for any measurable orbital cocycle $u: \mathscr{R}(\Gamma) \rightarrow G$ there exists a cocycle $\alpha \in Z^{1}(X \times \Gamma, G)$ such that $\alpha(x, \gamma)=u(\gamma x, x)$ [15].

For the freely acting group $\Gamma$, any cocycle $\alpha$ may be enlarged in a natural way to the full group $[\Gamma]$. Therefore, wherever convenient, we shall believe that $\alpha \in Z^{1}(X \times[\Gamma], G)$.

The two cocycles $\alpha$ and $\beta$ from $Z^{1}(X \times \Gamma, G)$ are called $\Gamma$-cohomologous, if there exists a measurable function $f: X \rightarrow G$ such that

$$
\alpha(x, \gamma)=f(\gamma x) \beta(x, \gamma) f(x)^{-1}
$$

A cocycle $\alpha$ is called a coboundary, if it is $\Gamma$-cohomologous to the unit cocycle, i.e. $\alpha(x, \gamma)=f(\gamma x) f(x)^{-1}$ for a measurable function $f: X \rightarrow G$.

1.4. Let the countable ergodic groups of automorphisms $\Gamma_{i} \subset \operatorname{Aut}\left(X_{i}\right.$, $\left.\mathcal{B}_{i}, \mu_{i}\right), i=1,2$ be orbital equivalent, i.e. let there exists a one-to-one map $\theta$ : $X_{1} \rightarrow X_{2}$ such that $\theta\left[\Gamma_{1}\right] \theta^{-1}=\left[\Gamma_{2}\right]$ and $\theta \circ \mu_{1} \sim \mu_{2}$. Let there be defined a cocycle $\beta \in Z^{1}\left(X_{2} \times\left[\Gamma_{2}\right], G\right)$ then, by the map $\theta$, the cocycle $\beta$ can be "transfered" to the group $\left[\Gamma_{1}\right]$ :

$$
\theta^{-1} \circ \beta\left(x_{1}, r_{1}\right)=\beta\left(\theta x_{1}, \theta r_{1} \theta^{-1}\right),\left(x_{1}, r_{1}\right) \in X_{1} \times\left[\Gamma_{1}\right]
$$

Relation (1.5) defines the one-to-one correspondence between the cocycles from $Z^{1}\left(X_{1} \times\left[\Gamma_{1}\right], G\right)$ and those from $Z^{1}\left(X_{2} \times\left[\Gamma_{2}\right], G\right)$. In this case, the $\Gamma_{1}$ cohomologous cocycles correspond to $\Gamma_{2}$-cohomologous cocycles and conversely. This is presented in more detail in [16].

We shall consider all the pairs $(\Gamma, \alpha)$, where $\Gamma$ is a countable ergodic group of automorphisms of $(X, \mathscr{B}, \mu), \alpha \in Z^{1}(X \times \Gamma, G)$ and define, on such the set of pairs, an equivalence relation generalizing the orbital equivalence of the groups of automorphisms. Then, we shall develop a complete system of invariants of such the equivalence relation.

Definition 1.2. Let there be the two pairs $\left(\Gamma_{i}, \alpha_{i}\right), i=1,2$, where $\Gamma_{i}$ is a freely acting group of automorphisms of $\left(X_{i}, \mathscr{B}_{i}, \mu_{i}\right)$ and $\alpha_{i} \in Z^{1}\left(X_{i} \times \Gamma_{i}, G\right)$. We shall call the pairs $\left(\Gamma_{1}, \alpha_{1}\right)$ and $\left(\Gamma_{2}, \alpha_{2}\right)$ weakly equivalent, if there exists $a$ map $\theta: X_{1} \rightarrow X_{2}$ which implies the orbital equivalence of $\Gamma_{1}$ and $\Gamma_{2}$ and is such that the cocycle $\theta^{-1} \circ \alpha_{2}$ is $\Gamma_{1}$-cohomologous to the cocycle $\alpha_{1}$.

If the cocycles $\theta^{-1} \circ \alpha_{2}$ and $\alpha_{1}$ are $\Gamma_{1}$-cohomologous, then the cocycles $\theta \circ \alpha_{1}$ and $\alpha_{2}$ are $\Gamma_{2}$-cohomologous. Thus, Definition 1.2 indeed suggests the equivalence relation on the set of pairs $(\Gamma, \alpha)$. 
If $\left(\Gamma, \alpha_{1}\right)$ and $\left(\Gamma, \alpha_{2}\right)$ are weakly equivalent, then the cocycles $\alpha_{1}$ and $\alpha_{2}$ will also be called weakly equivalent.

In the case $\alpha_{i}\left(x_{i}, \gamma_{i}\right)=\rho_{i}\left(x_{i}, r_{i}\right)$ Definition 1.2 coincides with the definition of the orbital equivalence of groups of automorphisms.

1.5. Consider on the group $\mathbb{Z}$ the Haar measure $\chi_{Z}$, i.e. $\chi_{Z}(i)=1, i \in \mathbb{Z}$. Denote by $\tau$ the shift on $\mathbb{Z}: \tau(i)=i+1$. Let $\Gamma \subset$ Aut $(X, \mathscr{B}, \mu)$ be a countable ergodic group of automorphisms and consider the direct product $\tilde{\Gamma}=\Gamma \times\left\{\tau^{n}\right.$ : $n \in \mathbb{Z}\} \subset$ Aut $\left(X \times \mathbb{Z}, \mu \times \chi_{\mathbb{Z}}\right)$. If $\alpha \in Z^{1}(X \times \Gamma, G)$, then define the cocycle $\tilde{\alpha}$ for $\tilde{\Gamma}: \tilde{\alpha}\left(x, n, r, \tau^{k}\right)=\alpha(x, \gamma)$, where $(x, n) \in X \times \mathbb{Z}, k \in \mathbb{Z}, r \in \Gamma$. Thus, $\tilde{\alpha} \in$ $\mathbb{Z}^{1}(X \times \mathbb{Z} \times \tilde{\Gamma}, G)$. The pair $(\tilde{\Gamma}, \widetilde{\alpha})$ will be called the countable expansion of $(\Gamma, \alpha)$.

Definition 1.3. Call the two pairs $\left(\Gamma_{1}, \alpha_{1}\right)$ and $\left(\Gamma_{2}, \alpha_{2}\right)$ stably weakly equivalent, if their countable expansions $\left(\tilde{\Gamma}_{1}, \widetilde{\alpha}_{1}\right)$ and $\left(\tilde{\Gamma}_{2}, \widetilde{\alpha}_{2}\right)$ are weakly equivalent.

Let $B \subset X(\mu B>0)$ and $\Gamma \subset$ Aut $(X, \mathscr{B}, \mu)$. Then, there exists in $[\Gamma]$ a countable group $\Gamma_{B}$, such that $\left[\Gamma_{B}\right]=[\Gamma]_{B}$, where $[\Gamma]_{B}=\{r \in[\Gamma]: \gamma x=x, x \in X$ $B$ \} [9]. If $\Gamma$ is of type III, then $\Gamma_{B}$ and $\Gamma$ are orbital equivalent and there exists a one-to-one measurable map $\theta: X \rightarrow B$ such that $\theta x \in \Gamma x$ for $\mu$-a.a. $x \in X$. If $\alpha \in Z^{1}(X \times \Gamma, G)$, then a cocycle $\alpha_{B} \in Z^{1}\left(X \times[\Gamma]_{B}, G\right)$ can be defined as $\alpha_{B}(x$, $\left.\gamma_{B}\right)=\alpha(x, \gamma),\left(x, \gamma_{B}\right) \in X \times \Gamma_{B}$, where $r \in \Gamma$ can be found from the condition $\gamma_{B} x=\gamma x$.

Proposition 1.4. Let there be a pair $(\Gamma, \alpha)$, where $\Gamma \subset$ Aut $(X, \mathscr{B}, \mu)$ and let $B \subset X, \mu(B)>0$. Then, (1) if $\Gamma$ is of type III or $\Gamma$ is of type $I I_{\infty}$ and $\mu(B)=$ $\infty$, then $(\Gamma, \alpha)$ and $\left(\Gamma_{B}, \alpha_{B}\right)$ are weakly equivalent; (2) if $\Gamma$ is of type $I I_{1}$ or $I I_{\infty}$ and $\mu(B)<\infty$, then $(\Gamma, \alpha)$ and $\left(\Gamma_{B}, \alpha_{B}\right)$ are stably weakly equivalent.

Proof. The proof is simple.

Corollary 1.5. If $\Gamma_{i}, i=1,2$ are groups of automorphisms of type III or II $I_{\infty}$, then the pairs $\left(\Gamma_{1}, \alpha_{1}\right)$ and $\left(\Gamma_{2}, \alpha_{2}\right)$ are weakly equivalent if and only if they are stably weakly equivalent.

If the group $\Gamma \subset$ Aut $(X, \mathscr{B}, \mu)$ is of type III, i.e. has the nontrivial RadonNikodym cocycle $\rho(x, \gamma)$, then it is natural to consider, along with the cocycle $\alpha \in Z^{1}(X \times \Gamma, G)$ the cocycle $\alpha_{0} \in Z^{1}(X \times \Gamma, G \times \mathbb{R})$ defined by the formula $\alpha_{0}(x, \gamma)=(\alpha(x, \gamma), \rho(x, \gamma))$.

Proposition 1.6. The pairs $\left(\Gamma_{1}, \alpha_{1}\right)$ and $\left(\Gamma_{2}, \alpha_{2}\right)$, where $\Gamma_{i} \subset$ Aut $\left(X_{i}, \mathcal{B}_{i}, \mu_{i}\right)$, 
$i=1,2$ are weakly equivalent if and only if the pairs $\left(\Gamma_{1}, \alpha_{1,0}\right)$ and $\left(\Gamma_{2}, \alpha_{2,0}\right)$ are weakly equivalent.

Proof. The proof is simple.

We notice that there is an example of the pairs, which are stably weakly equivalent, but are not weakly equivalent [2].

1.6. Consider in more detail the properties of the cocycles with values in an abelian 1.c.s. group $G$. By $\bar{G}=G \cup\{\infty\}$, we shall denote the one-point compactification of $G$.

Definition 1.7. Let $\Gamma$ be a countable ergodic group of automorphisms of $(X, \mathscr{B}, \mu)$ and $\alpha \in Z^{1}(X \times \Gamma, G)$. An element $f \in \bar{G}$ is called the essential value of the cocycle $\alpha$, if for any neighborhood $V_{f}$ of $f$ in $\bar{G}$ and any set $B \in \mathscr{B}, \mu(B)>0$ we have

$$
\mu\left(\bigcup_{\gamma \in \Gamma}\left(B \cap \gamma^{-1} B \cap\left\{x \in X: \alpha(x, \gamma) \in V_{f}\right\}\right)\right)>0 .
$$

The set of all essential values of the cocycle $\alpha$ will be denoted by $\bar{r}(\Gamma, \alpha)$. Put $r(\Gamma, \alpha)=\bar{r}(\Gamma, \alpha) \cap G$.

If $r(\Gamma, \alpha)=G$, then we shall say that the cocycle $\alpha$ has a dense range in the group $G$.

Lemma 1.8. [15] The following statements are true: (i) if the pairs $\left(\Gamma_{1}, \alpha_{1}\right)$ and $\left(\Gamma_{2}, \alpha_{2}\right)$ are weakly equivalent, then $\vec{r}\left(\Gamma_{1}, \alpha_{1}\right)=\bar{r}\left(\Gamma_{2}, \alpha_{2}\right)$; (ii) $r(\Gamma, \alpha)$ is a closed subgroup of $G$; (iii) a cocycle $\alpha$ is a coboundary if and only if $\vec{r}(\Gamma, \alpha)=\{0\}$.

Applying Lemma 1.8 to the pair $(\Gamma, \rho)$, where $\Gamma$ is a group of type III, we conclude that $r(\Gamma, \rho)$ can be only one of the following groups: $\{0\},\{n \log \lambda: n \in$ $\boldsymbol{Z}\}(0<\lambda<1)$ and $\boldsymbol{R}$. Accordingly, $\Gamma$ is called a type $\mathrm{III}_{0}, \mathrm{III}_{\lambda}$ and $\mathrm{III}_{1}$ group. The group $\Gamma$ is said to be of type $\mathrm{I}$, if the partition into its orbit is measurable.

Lemma 1.9. For any pair $(\Gamma, \alpha)$ we have $\bar{r}(\Gamma, \alpha)=\bar{r}(\tilde{\Gamma}, \tilde{\alpha})$.

Proof. Straightforward.

Since $r(\Gamma, \alpha)$ is a closed subgroup of $G$, then the quotient group $\hat{G}=G / r(\Gamma$, $\alpha)$ and the cocycle $\hat{\alpha} \in Z^{1}(X \times \Gamma, \hat{G})$ can be considered, setting

$$
\hat{\alpha}(x, \gamma)=\alpha(x, \gamma)+r(\Gamma, \alpha) .
$$

Lemma 1.10. [15] For any pair $(\Gamma, \hat{\alpha})$ always $r(\Gamma, \hat{\alpha})=\{\hat{0}\}$, where $\hat{0}$ is the identity in $\hat{G}$. 
Lemma 1.10 is the basis of the following definition.

Definition $\mathbb{1}$. 1 1. A cocycle $\alpha \in Z^{1}(X \times \Gamma, G)$ is called regular, if $\dot{r}(\Gamma, \hat{\alpha})=\{\hat{0}\}$ and nonregular, if $\vec{r}(\Gamma, \hat{\alpha})=\{\hat{0}, \infty\}$.

Lemma 1.12. [15] For any pair $(\Gamma, \alpha)$ the following conditions are equivalent: (i) $\alpha$ is a regular cocycle; (ii) $(\Gamma, \alpha)$ is weakly equivalent to $\left(\Gamma, \alpha_{1}\right)$, where a cocycle $\alpha_{1}(x, \gamma)$ takes values in $r(\Gamma, \alpha)$ for any $r \in \Gamma$ and a.a. $x \in X$.

Definition 1.13. Let $\Gamma$ be a freely acting conservative (i.e. not of type $\mathbb{I}$ ) group of automorphisms of $\left(X, \mathscr{B}_{,} \mu\right)$. A cocycle $\alpha \in Z^{1}(X \times \Gamma, G)$ is called recurrent, if for any set $B \in \mathscr{B}, \mu(B)>0$ and any neighborhood $V$ of the identity in $G$

$$
\mu\left(\bigcup_{\gamma \in \Gamma}\left(B \cap \gamma^{-1} B \cap\{x \in X: \alpha(x, \gamma) \in V\}\right)\right)>0 .
$$

If a cocycle $\alpha$ is not recurrent, then it is called transient.

Lemman $\mathbb{1}_{0} \mathbb{1} \hat{A}_{0}$ If $\alpha$ is a recurrent cocycle, then the cocycle $\hat{\alpha}$ defined according to (1.6) will also be recurrent.

Proof. Straightforward.

The following statement may be regarded as another definition of the transient cocycles.

Proposition 1.15. [15] A cocycle $\alpha \in Z^{1}(X \times \Gamma, G)$ is transient if and only if there exists a measurable set $B_{0} \subset X, \mu\left(B_{0}\right)>0$ and there is a neighborhood $V_{0}$ of the identity in $G$ both such that

$$
\mu\left(\bigcup_{\gamma \in \Gamma}\left(B_{0} \cap r^{-1} B_{0} \cap\left\{x \in X: \alpha(x, \gamma) \in V_{0}\right\}\right)\right)=0
$$

It follows from (1.7) that for the group $\Gamma_{B_{0}} \subset[\Gamma]$ the cocycle $\alpha_{B_{0}}$ does not take the values in $V_{0}$.

For a transient cocycle $\alpha \in Z^{1}(X \times \Gamma, G)$ always $\bar{r}(\Gamma, \alpha)=\{0, \infty\}$.

Defimition 1.16. A cocycle $\alpha \in Z^{1}(X \times \Gamma, G)$ is called lacunary, if there exists a neighborhood $V_{0}$ of the identity in $G$ such that

$$
\mu\left(\bigcup_{\gamma \in \Gamma}\left\{x \in X: \alpha(x, \gamma) \in V_{0}-\{0\}\right)=0 .\right.
$$

Lemma 1.17\% [15] A cocycle $\alpha \in Z^{1}(X \times \Gamma, G)$ is cohomologous to a lacunary cocycle, if and only if there exists a neighborhood $V_{1}$ of the identity in $G$ such that $r(\Gamma, \alpha) \cap V_{\mathbb{I}}=\{0\}$. 


\section{§2. Associated Actions and Transient Cocycles}

2.1. Everywhere in this section we shall assume that $\Gamma$ is a freely acting ergodic group of automorphisms of a Lebesgue space $(X, \mathcal{B}, \mu), G$ an arbitrary 1.c.s. group, and a cocycle $\alpha \in Z^{1}(X \times \Gamma, G)$. By $\chi_{G}$ the Haar measure on the group $G$ will be denoted.

Let $\Gamma(\alpha) \subset$ Aut $\left(X \times G, \mu \times \chi_{G}\right)$ be the skew product constructed by the group $\Gamma$ and the cocycle $\alpha$ : for $\gamma(\alpha) \in \Gamma(\alpha),(x, g) \in X \times G$

$$
r(\alpha)(x, g)=(r x, \alpha(x, r) g), \quad r \in \Gamma .
$$

Lemma 2.1. [15] $\Gamma(\alpha)$ is conservative if and only if $\alpha$ is a recurrent cocycle, and $\Gamma(\alpha)$ is of type $I$ if and only if $\alpha$ is a transient cocycle.

It follows from Lemma 2.1 that the properties of recurrence and transientness of cocycles are invariants of weak equivalence.

Define an action $V$ of the group $G$ on $\left(X \times G, \mu \times \chi_{G}\right)$ :

$$
V(g)(x, h)=\left(x, h g^{-1}\right), \quad g \in G .
$$

It follows from (2.1) and (2.2) that the groups of automorphisms $\Gamma(\alpha)$ and $V(G)$ commutate elementwise. Let $\xi$ be the measurable hull of partition into the orbits of $\Gamma(\alpha)$. Then, the group $V(G)$ generates on the quotient space $(\Omega, \nu)=\left((X \times G) / \xi,\left(\mu \times \chi_{G}\right) / \xi\right)$ a new action of $G$ which will be denoted by $W_{(\Gamma, \alpha)}(G)$ or just $W(G)$.

Definition 2.2. The action $W_{(r, \infty)}(G)$ of the group $G$ is called the action associated with the pair $(\Gamma, \alpha)$ or the Mackey action.

Proposition 2.3. If the pairs $\left(\Gamma_{1}, \alpha_{1}\right)$ and $\left(\Gamma_{2}, \alpha_{2}\right)$ are weakly equivalent, then the associated actions $W_{\left(\Gamma_{1}, \alpha_{1}\right)}(G)$ and $W_{\left(\Gamma_{2}, \alpha_{2}\right)}(G)$ are isomorphic.

Proof. It follows from the above condition that there exists a one-to-one measurable map $\varphi: X_{1} \rightarrow X_{2}$ such that $\varphi\left[\Gamma_{1}\right] \varphi^{-1}=\left[\Gamma_{2}\right], \varphi^{-1} \circ \mu_{2} \sim \mu_{1}$ and for $\mu_{1}$-a.a. $x \in X_{1}$

$$
\alpha_{2}\left(\varphi x, \varphi r_{1} \varphi^{-1}\right)=f\left(r_{1} x\right) \alpha_{1}\left(x, r_{1}\right) f(x)^{-1}, \quad r_{1} \in\left[\Gamma_{1}\right] \text {, }
$$

where $\Gamma_{i} \subset$ Aut $\left(X_{i}, \mathscr{B}_{i}, \mu_{i}\right), i=1,2$ and $f: X_{1} \rightarrow G$ is a measurable map. Define

$$
\Phi(x, h)=(\varphi x, f(x) h), \quad(x, h) \in X_{1} \times G .
$$

Therefore, the quotient map $\tilde{\Phi}:\left(\Omega_{1}, \nu_{1}\right) \rightarrow\left(\Omega_{2}, \nu_{2}\right)$ satisfies the equality $\tilde{\Phi} W_{\left(\Gamma_{1}, \alpha_{1}\right)}(g) \tilde{\Phi}^{-1}=W_{\left(\Gamma_{2}, \alpha_{2}\right)}(g), g \in G$. 
Proposition 2.4. (1) Let $(\tilde{\Gamma}, \tilde{\alpha})$ be a countable expansion of a pair $(\Gamma, \alpha)$. Then, $W_{(\tilde{\Gamma}, \tilde{\alpha})}(G)$ is isomorphic to $W_{(\Gamma, \alpha)}(G)$. (2) If the pairs $\left(\Gamma_{1}, \alpha_{1}\right)$, and $\left(\Gamma_{2}\right.$, $\left.\alpha_{2}\right)$ are stably weakly equivalent, then the associated actions $W_{\left(\Gamma_{1}, \alpha_{1}\right)}(G)$ and $W_{\left(r_{2}, \alpha_{2}\right)}(G)$ are isomorphic.

Proof. Straightforward.

2.2. Below we shall consider the situation, where a cocycle $\alpha$ from a pair $(\Gamma, \alpha)$ is transient and takes the values in the 1.c.s. group $G$.

It follows from Lemma 2.1 that the partition into orbits of the group $\Gamma(\alpha)$ $\subset$ Aut $\left(X \times G, \mu \times \chi_{G}\right)$ is measurable. Then, the quotient space $\Omega$ can be regarded as a measurable subset of positive measure in $X \times G$, which intersect with each orbit of $\Gamma(\alpha)$ exactly at one point. Then, $X \times G=\cup_{\gamma \in \Gamma} \gamma(\alpha) \Omega$ and the measure $\nu=\left.\left(\mu \times \chi_{G}\right)\right|_{\Omega}$. The action $W(G)=W_{(\Gamma, \alpha)}(G)$ associated with $(\Gamma, \alpha)$ will be written as follows. Let $(x, h) \in \Omega, g \in G$, then

$$
W(g)(x, h)=r(\alpha)\left(x, h g^{-1}\right),
$$

where $r(\alpha)$ is an element of $\Gamma(\alpha)$ such that $\left(x, h g^{-1}\right) \in \gamma(\alpha)^{-1} \Omega$.

The further arguments are convenient to be made in terms of the measurable groupoid theory (see [3] and [12]). Denote by $\mathscr{H}$ a measurable groupoid with discrete orbits which is defined by the action of $\Gamma$ on $X$. Any cocycle $\alpha \in$ $Z^{1}(X \times \Gamma, G)$ will define a homomorphism of the groupoid $\mathcal{H}$ into the group $G$. Denote by $\mathcal{G}$ a measurable groupoid with continuous orbits generated by the group $W(G)$ of automorphisms of $\Omega$.

According to Proposition 1.15, for a transient cocycle $\alpha$ there exists in $X$ a measurable subset $B(\mu(B)>0)$ for which $\alpha\left(x, \gamma_{B}\right) \notin V_{0}$, where $x \in B, \gamma_{B} \in[\Gamma]_{B}$ and $V_{0}$ is a neighborhood of the identity in $G$. Consider the pair $\left(\Gamma_{B}, \alpha_{B}\right)$ and let $\mathcal{G}_{B}$ denote the measurable groupoid generated by the action $W_{B}(G)=$ $W_{\left(\Gamma_{B}, \alpha_{B}\right)}(G)$ associated with $\left(\Gamma_{B}, \alpha_{B}\right)$, which was defined on the quotient space $\left(\Omega_{B}, \nu_{B}\right), \Omega_{B} \subset B \times G$.

Recall the definition of a return cocycle. Let $U(H)$ be a group of Borel automorphisms of $(X, \mathscr{B}, \mu)$ (not necessarily countable), which is a free Borel action of a 1.c.s. group $H$. A set $E \subset X$ is called a complete lacunary section for $U(H)$ if $\mu(X-U(H) E)=0$ and there is a neighborhood $V$ of the identity of $H$ such that $U(V) x \cap E=\{x\}$ for all $x \in E$ [3]. There arises on $E$ a countable measurable equivalence relation $\mathcal{R}$ and hence so does a group $\Gamma$ of automorphisms of the set $E$ generating the equivalence relation $R$. Put $u(y, x)=h$ for $(y, x) \in \mathscr{R}$, if $U(h) x=y$. Since $U(H)$ acts freely, the orbital cocycle $u$ is defined 
uniquely.

Definition 2.5. The cocycle $u: \mathcal{R} \rightarrow H$ constructed in the above way is called a return cocycle (or homomorphism) for the action $U(H)$ with respect to the set $E$ (or in short, a return cocycle on $E$ ).

If $\mathcal{G}$ is a measurable groupoid and $E$ a subset of its set of units, then $\left.\mathcal{G}\right|_{E}$ will denote the reduction of the groupoid $\mathcal{G}$ on $E$.

The following lemma is a slightly modified version of Lemma 7.4 of [3].

Lemma 2.6. Let the groupoids $\mathcal{G}, \mathcal{H}$ and $\mathcal{G}_{B}$ be as above, $\alpha: \mathcal{H} \rightarrow G$ be a transient homomorphism, and $\alpha_{B} \notin V_{0}$, where $V_{0}$ is a neighborhood of the identity in G. There exists a canonical isomorphism of the groupoid $\left.\mathcal{H}\right|_{B}=B \times \Gamma_{B}$ onto the groupoid $\left.\mathcal{G}_{B}\right|_{E}$, where $E$ is a complete lacunary section of the action $W_{\left(\Gamma_{B}, \alpha_{B}\right)}(G)$ $=W_{B}(G)$ on $\Omega_{B}$, such that for this isomorphism the homomorphism $\alpha_{B}: \mathcal{H}_{B} \rightarrow$ $G$ transforms into the return homomorphism of the action $W_{B}(G)$ on $E$,

Proof. Let us introduce a measurable map $\theta: B \rightarrow \Omega_{B}$ putting $\theta x=(y, h)$, if there exists an automorphism $\gamma_{B}\left(\alpha_{B}\right) \in \Gamma_{B}\left(\alpha_{B}\right)$ depending on the point $x \in B$, for which $\gamma_{B}\left(\alpha_{B}\right)(x, e)=(y, h)$. Prove that $\theta(B)$ is a complete lacunary section for $W_{B}(G)$ on $\left(\Omega_{B}, \nu_{B}\right)$. Indeed, since $B \times\{e\}$ intersects every orbit of the group $V(G)$, then $\theta(B)$ will also intersects every orbit of the group $W_{B}(G)$, which means completeness of the section $\theta(B)$. Describe now the equivalence relation which generates on $\theta(B)$ the associated action $W_{B}(G)$. If $y=r x$, where $x, y \in B$, $r \in[\Gamma]_{B}$, then we shall show that

$$
\theta(y)=W_{B}\left(\alpha_{B}(x, \gamma)\right) \theta(x) .
$$

Let $\theta(x)=\left(r_{1} x, \alpha_{B}\left(x, r_{1}\right)\right), \theta(y)=\left(r_{2} y, \alpha\left(y, r_{2}\right)\right), r_{1}, r_{2} \in[\Gamma]_{B}$. Then,

$$
W_{B}\left(\alpha_{B}(x, r)\right) \theta(x)=r_{0}\left(\alpha_{B}\right)\left(\gamma_{1} x, \alpha_{B}\left(x, \gamma_{1}\right) \alpha_{B}(x, \gamma)^{-1}\right),
$$

where the automorphism $\gamma_{0}\left(\alpha_{B}\right)$ is chosen from the condition $\gamma_{0}\left(\alpha_{B}\right)\left(r_{1} x, \alpha_{B}\left(x, r_{1}\right) \alpha_{B}\left(x, \gamma^{-1}\right)\right) \in \Omega_{B}$. It is easy to see that the role $r_{0}$ can be played by $r_{2} r r_{1}^{-1}$. Indeed,

$$
r_{2} r r_{1}^{-1}\left(\alpha_{B}\right)\left(r_{1} x, \alpha_{B}\left(x, r_{1}\right) \alpha_{B}(x, r)^{-1}\right)=\left(r_{2} y, \alpha_{B}\left(y_{1}, r_{2}\right)\right)=\theta(y),
$$

By comparing (2.8) and (2.7), we conclude that (2.6) is true.

Vice versa, if $W_{B}(h) \theta(x)=\theta(y)$, then similar arguments can show that there exists an automorphism $r \in[\Gamma]_{B}$ for which $r x=y$ and

$$
h=\alpha_{B}(x, \gamma) \text {. }
$$


Thus, it is proved that the orbits of the equivalence relation on $\theta(B)$ are countable, because the cocycle $\alpha_{B} \notin V_{0}$. Therefore, the section $\theta(B)$ of the action $W_{B}(G)$ is lacunary. Besides, it follows from (2.6) and (2.9) that it is the cocycle $\alpha_{B}$ that corresponds to the return cocycle of the action $W_{B}(G)$ on $\theta(B)$. Consider the measure $\theta \circ \mu$ on $\theta(B)$, and let $E$ be a Borel subset of $\theta(B)$ which is full with respect to $\theta \circ \mu$. Because the later is zero on sets from $\theta(B)$ if and only if the measure $\mu$ is zero on the respective sets from $B$, then $\theta \circ \mu$ is equivalent to the projection of the measure $\left.\left(\mu \times \chi_{G}\right)\right|_{\Omega_{B}}$ on the section $E$ along the orbits of $W_{B}(G)$. Therefore, the map $(y, x) \rightarrow(\theta(y), \theta(x))$ is the desired isomorphism of the groupoids $\left.\mathcal{H}\right|_{B}$ and $\left.\mathcal{G}_{B}\right|_{E}$.

Remark 2.7. Proposition 1.4, 2.3 and 2.4 imply that for any group $\Gamma \subset$ Aut $(X, \mathscr{B}, \mu)$ and any $B \subset X(\mu(B)>0)$ the associated actions $W_{(\Gamma, \alpha)}(G)$ and $W_{\left(\Gamma_{B}, \alpha_{B}\right)}(G)$ are isomorphic. Therefore, everywhere in this section we shall denote the associated action by $W(G)$.

Lemma 2.8. Let $W(G)$ be a free non-singular action of a l.c.s. group $G$ on $(\Omega, \nu)$. Let also $E_{1}$ and $E_{2}$ be complete sections of the action $W(G)$ on $\Omega$ and $\mathcal{G}_{\mathbb{1}}$ $=\left.\mathcal{G}\right|_{E_{1}}, \mathcal{G}_{2}=\left.\mathcal{G}\right|_{E_{2}}$ be the reductions of the groupoid $\mathcal{G}=\Omega \times W(G)$ on $E_{1}$ and $E_{2}$. Assume that there exists an isomorphism $\psi_{0}: \mathcal{G}_{1} \rightarrow \mathcal{G}_{2}$ and let $\psi_{0}$ be the restriction of the map $\psi$ onto $E_{1}$, i.e. $\psi_{0}: E_{1} \rightarrow E_{2}$. If $\psi_{0}$ is an inner automorphism of the groupoid $\mathcal{G}$, i.e. the points $x$ and $\psi_{0}(x)$ lie in one and the same orbit of $W(G)$, then the return homomorphisms $\pi_{1}: \mathcal{G}_{1} \rightarrow G$ and $\psi^{-1} \circ \pi_{2}: \mathcal{G}_{1} \rightarrow G$ are equivalent (or in other words, the cocycles $\pi_{1}$ and $\psi^{-1} \circ \pi_{2}$ are cohomologous).

Proof. Define the homomorphism $\pi: \mathcal{G} \rightarrow G$ with putting for $(\omega, \mathbb{W}(h)) \in$ $\mathcal{G}(\omega \in \Omega)$

$$
\pi(\omega, \mathbb{W}(h))=h
$$

Then the return homomorphisms $\pi_{1}$ and $\pi_{2}$ are related to $\pi$ as follows

$$
\pi_{1}=\pi / \mathcal{G}_{1}, \quad \pi_{2}=\pi / \mathcal{G}_{2} .
$$

By the condition of the lemma, the points $x$ and $\psi_{0}(x), x \in E_{1}$ are related as $\psi_{0}(x)=W(h) x$, where $h=h(x) \in G$. The measurability of the function $h(x)$ follows from the measurability of the maps $x \rightarrow \psi_{0}(x),(x, h) \rightarrow W(h) x$. By the definition of the cocycle $\psi^{-1} \circ \pi_{2}$, we have the following for $(x, \mathbb{W}(g)) \in \mathcal{G}_{1}$ :

$$
\psi^{-1} \circ \pi_{2}(x, W(g))=h(W(g) x) g h(x)^{-1},
$$

because $\psi_{0} W(g) \psi_{0}^{-1}\left(\psi_{0}(x)\right) \in E_{2}$. Formula (2.12) can be written, according to 
(2.10) and (2.11), as $\psi_{0}^{-1} \circ \pi_{2}(x, W(g))=h(W(g) x) \pi_{1}(x, W(g)) h(x)^{-1}$, whence follows the statement of the lemma.

2.3. If $\Gamma$ is a countable group of automorphisms of $(X, \mathscr{B}, \mu)$ and a cocycle $\alpha \in Z^{1}(X \times \Gamma, G)$, then the pair $(\Gamma, \alpha)$ corresponds to the pair $(\mathcal{H}, \alpha)$, where $\mathcal{H}$ is a measurable groupoid generated by the action $\Gamma$ on $X$ and $\alpha$ a homomorphism from $\mathscr{H}$ into $G$. The two pairs $\left(\mathscr{H}_{1}, \alpha_{1}\right)$ and $\left(\mathscr{H}_{2}, \alpha_{2}\right)$ will be weakly equivalent, if there exists an isomorphism $\varphi$ of the groupoids $\mathscr{H}_{1}$ and $\mathscr{H}_{2}$ such that the homomorphisms $\alpha_{1}$ and $\varphi^{-1} \circ \alpha_{2}$ are equivalent. (The definition of equivalent homomorphisms see e.g. in [3]).

Theorem 2.9. Let there be defined the two pairs $\left(\mathscr{H}_{1}, \alpha_{1}\right)$ and $\left(\mathscr{H}_{2}, \alpha_{2}\right)$, where $\mathscr{H}_{i}$ is a discrete measurable groupoid generated by an action of the group $\Gamma_{i} \subset$ $\operatorname{Aut}\left(X_{i}, \mathscr{B}_{i}, \mu_{i}\right)$ and $\alpha_{i}$ is transient homomorphism from $\mathscr{H}_{i}$ into $G, i=1,2$. Then, if the associated actions $W_{1}(G)=W_{\left(\mathcal{H}_{1}, \alpha_{1}\right)}(G)$ and $W_{2}(G)=W_{\left(\mathcal{H}_{2}, \alpha_{2}\right)}(G)$ are isomorphic, then the pairs $\left(\mathcal{H}_{1}, \alpha_{1}\right)$ and $\left(\mathcal{H}_{2}, \alpha_{2}\right)$ are stably weakly equivalent.

Proof. Since $\alpha_{i}(i=1,2)$ is a transient homomorphism, then there exists a set $B_{i}$ and a neighborhood $V_{0}$ of the identity in $G$, such that $\alpha_{i}\left(x_{i}, \gamma_{i}\right) \notin V_{0}$ for $\left(x_{i}\right.$, $\left.\gamma_{i}\right)\left.\in \mathcal{H}_{i}\right|_{B_{i}}, i=1,2$. We shall consider the pairs $\left(\left.\mathcal{H}_{1}\right|_{B_{1}}, \alpha_{1 B_{1}}\right)$ and $\left(\left.\mathcal{H}_{2}\right|_{B_{2}}, \alpha_{2 B_{2}}\right)$, Let $W_{i B_{i}}(G),\left.\mathscr{H}_{i}\right|_{B_{i}},\left.\mathcal{G}_{i}\right|_{B_{i}}$ and $\left(\Omega_{B_{i}}, \nu_{B_{i}}\right), i=1,2$ be as in Lemma 2.6. It follows from the condition of the theorem and Proposition 2.4 that there exists a oneto-one measurable map $\psi: \Omega_{B_{1}} \rightarrow \Omega_{B_{2}}$ such that $\psi \circ \nu_{B_{1}} \sim \nu_{B_{2}}$ and

$$
\psi W_{1 B_{1}}(g)=W_{2 B_{2}}(g) \psi, \quad g \in G,
$$

where $W_{i B_{i}}(G)$ is the action of $G$ associated with $\left(\left.\mathcal{H}_{i}\right|_{B_{i}}, \alpha_{i B_{i}}\right), i=1,2$. In view of (2.13) the isomorphism $\Psi$ of the groupoids $\left.\mathcal{G}_{1}\right|_{B_{1}}=\mathcal{G}_{B_{1}}$ and $\left.\mathcal{G}_{2}\right|_{B_{2}}=\mathcal{G}_{B_{2}}$ is defined by the map

$$
\Psi:((x, h), g)=(\psi(x, h), g), \quad(x, h) \in \Omega_{B_{1}} .
$$

By applying Lemma 2.6, we find that there exist canonical isomorphisms $j_{1}$ : $\left.\left.\mathscr{H}_{1}\right|_{B_{1}} \rightarrow \mathcal{G}_{B_{1}}\right|_{E_{1}}$ and $j_{2}:\left.\left.\mathscr{H}_{2}\right|_{B_{2}} \rightarrow \mathcal{G}_{B_{2}}\right|_{E_{2}}$, where $E_{i}$ is a complete lacunary section for $W_{i B_{i}}(G)$ such that $j_{i} \circ \alpha_{i B_{i}}$ is a return cocycle for restriction of $W_{i B_{i}}(G)$ on $E_{i}$, $i=1,2$. From relation (2.14) it follows that the map $\Psi$ also defines the isomorphism of the groupoids $\left.\mathcal{G}_{B_{1}}\right|_{E_{1}}$ and $\left.\mathcal{G}_{B_{2}}\right|_{\psi\left(E_{1}\right)}$.

Put $\left.\tilde{\mathscr{H}}_{i}\right|_{B_{i}}=\left.\mathscr{H}_{i}\right|_{B_{i}} \times Z$, where $Z=\mathbb{Z} \times \mathbb{Z}$ is the transitive discrete groupoid generated by the shift on $\mathbb{Z}$. Consider two groupoids with the discrete orbits $\left.\mathcal{G}_{B_{2}}\right|_{E_{2}} \times Z$ and $\left.\mathcal{G}_{B_{2}}\right|_{\psi\left(E_{1}\right)} \times Z$. In view of Theorem 4.12 of [3], there exists an isomorphism $\tau$ can be inner for the groupoid $\mathcal{G}_{B_{2}}$. Let $J=[0,1] \times[0,1]$ be tran- 
sitive groupoid with continuous orbits. It follows from [3, Corollary 4.4] that the groupoid $\mathcal{G}_{B_{2}}$ is isomorphic to the groupoid $\left.\mathcal{G}_{B_{2}}\right|_{E_{2}} \times Z \times J$ and to keep the notation simple, we shall assume the groupoids to coincide. Putting $\tilde{\tau}=\tau \times \mathbb{1} \times$ $\mathbb{1}$ enlarge $\tau$ to the automorphism $\tilde{\tau}$ of $\mathcal{G}_{2}$. By Theorem 1 of [7], there exists an automorphism $q$ inner for $\mathcal{G}_{B_{2}}$ and such that $q \circ \tilde{\tau}=a \times \mathbb{1}$, where $a$ is an automorphism of the groupoid $\left.\mathcal{G}_{B_{2}}\right|_{E_{2}} \times Z$. With account of the equalities

$$
\left.q \circ \tilde{\tau}\right|_{\left.\mathcal{G}_{B_{2}}\right|_{H_{2}} \times Z}=\left.q \circ \tau\right|_{\left.\mathcal{G}_{B_{2}}\right|_{H_{2}} \times Z}=a
$$

we find that $q$ maps $\left.\mathcal{G}_{B_{2}}\right|_{\psi\left(E_{1}\right)} \times Z$ into $\left.\mathcal{G}_{B_{2}}\right|_{E_{2}} \times Z$. Thus, it is proved that for the groupoid $\mathcal{G}_{B_{2}}$ there are two lacunary sections such that the groupoids $\left.\mathcal{G}_{B_{2}}\right|_{\psi\left(E_{1}\right)} \times Z$ and $\left.\mathcal{G}_{B_{2}}\right|_{E_{2}} \times Z$ defined on them are isomorphic, this isomorphism being inner with respect to $\mathcal{G}_{B_{2}}$. It follows from Lemma 2.6, that the homomorphisms $j_{2} \circ \tilde{\alpha}_{2 B_{2}}$ and $\Psi \circ j_{1} \circ \tilde{\alpha}_{1 B_{1}}$ are return ones for the groupoids $\left.\mathcal{G}_{B_{2}}\right|_{E_{2}} \times Z$ and $\left.\mathcal{G}_{B_{2}}\right|_{\psi\left(E_{1}\right)} \times Z$ (here $\tilde{\alpha}_{i_{B_{i}}}, i=1,2$ is the countable expansion of the cocycle $\alpha_{i B_{i}}$ ). According to Lemma 2.8, the homomorphisms $j_{2} \circ \widetilde{\alpha}_{2 B_{2}}$ and $\psi \circ j_{1} \circ \widetilde{\alpha}_{1 B_{1}}$ are equivalent. It now remains to note that $\varphi=j_{2}^{-1} \circ q \circ \Psi \circ j_{1}$ is the isomorphism of the groupoids $\left.\widetilde{\mathcal{H}}_{1}\right|_{B_{1}}$ and $\left.\mathcal{H}_{2}\right|_{B_{2}}$, and therefore the pairs $\left(\mathcal{H}_{1}, \alpha_{1}\right)$ and $\left(\mathcal{H}_{2}, \alpha_{2}\right)$ are stably weakly equivalent.

Remark. The isomorphism $\tau$ (the subject of the proof of Theorem 2.9) may be directly chosen to be inner, as is obvious from simple considerations.

Our proof of Theorem 2.9 is similar in the idea to that in [7]; another proof was given in [2].

2.4. Here we shall consider the property of transientness for the cocycle $\alpha_{0}=(\alpha, \rho)$.

Proposition 2.10. Let $\Gamma$ be a countable group of automorphisms of $(X, \mathcal{B}$, $\mu)$. A cocycle $\alpha_{0} \in Z^{1}(X \times \Gamma, G \times \mathbb{R})$ is transient if and only if the cocycle $\alpha \in$ $Z^{1}(X \times \Gamma, G)$ is transient.

Proof. Let $\alpha$ be a transient cocycle. Consider the group of automorphisms $\Gamma_{d} \subset$ Aut $\left(X \times \boldsymbol{R}, \mu \times \chi_{\boldsymbol{R}}\right)$ dual to $\Gamma$, whose elements act as $\gamma_{d}(x, u)=(\gamma x$, $u+\rho(x, \gamma)), r \in \Gamma$ i.e. $\Gamma_{d}=\Gamma(\rho)$. The cocycle $\alpha$ is enlarged to the cocycle $\alpha^{\prime}$ of the group $\Gamma_{d}$ by the formula $\alpha^{\prime}\left(x, u, \gamma_{d}\right)=\alpha(x, \gamma)$. The transientness of $\alpha$ means transientness of $\alpha^{\prime}$. Therefore, by Lemma 2.1, the partition into orbits of the group $\Gamma_{d}\left(\alpha^{\prime}\right) \subset$ Aut $\left(X \times \mathbb{R} \times G, \mu \times \chi_{\mathbb{R}} \times \chi_{G}\right)$ is measurable. As is easy to see, $\Gamma_{d}\left(\alpha^{\prime}\right)$ and $\Gamma\left(\alpha_{0}\right)$ coincide. By applying again Lemma 21 , we find that $\alpha_{0}$ is transient. Conversely, let $\alpha_{0}$ be a transient cocycle. This means that $\Gamma\left(\alpha_{0}\right)$ is 
of type I group of automorphisms. It follows from the equality $\Gamma\left(\alpha_{0}\right)=\Gamma(\alpha)_{d}$ that the group $\Gamma(\alpha)_{d}$ is also of type I; but then it is evident that in this case the group $\Gamma(\alpha) \subset$ Aut $\left(X \times G, \mu \times \chi_{G}\right)$ can only be of type I as well. Therefore, the cocycle $\alpha$ is transient.

Corollary 2.11. Let the pairs $\left(\mathcal{H}_{1}, \alpha_{1}\right)$ and $\left(\mathcal{H}_{2}, \alpha_{2}\right)$ be as in Theorem 2.9. Then, for $G_{0}=G \times \boldsymbol{R}:$ (i) if the associated actions $W_{\left(\mathcal{H}_{1},\left(\alpha_{1}\right)_{0}\right)}\left(G_{0}\right)$ and $W_{\left(\mathscr{H}_{2},\left(\alpha_{2}\right)_{0}\right)}\left(G_{0}\right)$ are isomorphic. then $\left(\mathcal{H}_{1}, \alpha_{1}\right)$ and $\left(\mathcal{H}_{2}, \alpha_{2}\right)$ are stably weakly equivalent; (ii) if $W_{\left(\mathcal{H}_{1}, \alpha_{1}\right)}(G)$ and $W_{\left(\mathcal{H}_{2}, \alpha_{2}\right)}(G)$ are isomorphic, then so are the actions $W_{\left(\mathcal{H}_{1},\left(\alpha_{1}\right)_{0}\right)}\left(G_{0}\right)$ and $W_{\left(\mathcal{H}_{2},\left(\alpha_{2}\right)_{0}\right)}\left(G_{0}\right)$.

\section{§3. Measurable Fields of Cocycles}

3.1. In this and next sections we shall only consider the cocycles $\alpha$ which take values in an abelian 1.c.s. group $G$. However the findings are valid in more general situation (see the end of this section).

Now we shall construct, for any closed subgroup $H_{0}$ of $G_{0}=G \times \boldsymbol{R}$, an approximately finite (a.f.) ergodic group of automorphisms $\Delta \subset$ Aut $\left(Y_{0}, p\right)$ and a cocycle $\beta$, both such that $\beta_{0}=(\beta, \rho)$ takes the values in $H_{0}$ and $r\left(\Delta, \beta_{0}\right)=H_{0}$.

Let $\left\{h_{0}(n)\right\}_{n=1}^{\infty}$ be a dense sequence of group elements from $H_{0}$, such that every member of this sequence occurs in it an infinite number of times. Every element $h_{0}(n)$ can be represented as $\left(h_{G}(n), h_{R}(n)\right), n \in N$. Choose $\left\{h_{0}(n)\right\}_{n=1}^{\infty}$ to belong in turn to a countable subgroup $H_{0}^{\prime}$ (dense in $H_{0}$ ). The projections of the groups $H_{0}$ and $H_{0}^{\prime}$ onto $G$ and $\boldsymbol{R}$ will be denoted by $H_{G}, H_{\boldsymbol{R}}$ and $H_{G}^{\prime}, H_{\boldsymbol{R}}^{\prime}$ respectively. The group $H_{0}$ and its projections $H_{G}$ and $H_{\boldsymbol{R}}$ can be either discrete or continuous. The closed $H_{0}$, generally speaking, does not mean that $H_{G}$ and $H_{\boldsymbol{R}}$ are closed.

Put $Y_{0}=\{0,1\}^{N}$ and $A_{n}(0)=\left\{y \in Y_{0}: y_{n}=0\right\}, A_{n}(1)=\left\{y \in Y_{0}: y_{n}=1\right\}, n \in$ $N$, where $y \in Y_{0}$ is $\left\{y_{n}\right\}_{n=1}^{\infty}$. Then, $A_{n}(0) \cap A_{n}(1)=\varnothing$ and $A_{n}(0) \cup A_{n}(1)=Y_{0}$, $n \in \boldsymbol{N}$. Consider on $Y_{0}$ the probability product-measure $p$, for which

$$
p\left(A_{n}(1)\right)=\exp \left(h_{R}(n)\right) p\left(A_{n}(0)\right),
$$

where the sequence $\left\{h_{0}(n)=\left(h_{G}(n), h_{R}(n)\right)\right\}_{n=1}^{\infty}$ has been chosen above. Introduce the automorphisms $\delta_{n} \in \operatorname{Aut}\left(Y_{0}, p\right), n \in N$ such that $\delta_{n}\left\{y_{k}\right\}=\left\{y_{k}^{\prime}\right\}$, where $y_{k}^{\prime}=$ $y_{k}$ with $k \neq n$ and $y_{n}^{\prime}=y_{n}+1(\bmod 2)$. It follows from (3.1) that

$$
\rho\left(y, \delta_{n}\right)=h_{R}(n), y \in A_{n}(0), n \in N .
$$

Denote by $\Delta$ the group of automorphisms of $\left(Y_{0}, p\right)$ generated by $\delta_{n}, n \in \boldsymbol{N}$. 
As is known, $\Delta$ is ergodic and a.f. [9]. If $H_{R}^{\prime}$ is a dense subgroup of $\mathbb{R}$, then $\Delta$ is of type $\mathbb{I I I}_{1}$; if $H_{\mathbb{R}}^{\prime}=\{n \log \lambda: h \in \mathbb{Z}\}$, then $\Delta$ is of type $\mathbb{H I I}_{\lambda}(0<\lambda<1)$; and if $H_{R}^{\prime}=\{0\}$ (i.e. $\left.H_{0} \subset G\right)$, then $\Delta$ is of type $\mathbb{H}_{\mathbb{l}}$. Consider the case of the type $\mathbb{I I I}$ group $\Delta$; type $\mathbb{I I}$ group $\Delta$ is considered similarly.

Define the cocycle $\beta \in Z^{1}\left(Y_{0} \times \Delta, H_{G}\right)$ on the generators of the group $\Delta$ :

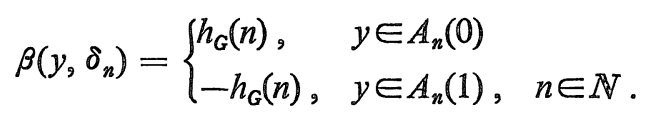

From (3.2) and (3.3) it follows that

$$
\beta_{0}\left(y, \delta_{n}\right)=h_{0}(n), \quad y \in A_{n}(0), \quad n \in \mathbb{N} .
$$

Lemma 3.1. Let $\Delta_{0}$ be the subgroup of [U] specified as $\Delta_{0}=\left\{\gamma \in[\Delta]: \beta_{0}(y, \gamma)\right.$ $=0\}$. Then, $\Delta_{0}$ is the ergodic group of automorphisms of $\left(Y_{0}, p\right)$.

Proof. By the choice of the sequence $\left\{h_{0}(n)\right\}_{n=1}^{\infty}$, there are infinitely many numbers $n$ and $n_{1}$, such that $h_{0}(n)=h_{0}\left(n_{1}\right)$. Put for such $n$ and $n_{1}$

$$
\left(r\left(n, n_{1}\right) y\right)_{k}= \begin{cases}y_{n_{1}}, & k=n \\ y_{n}, & k=n_{1} \\ y_{k}, & k \neq n, n_{1}\end{cases}
$$

From (3.2), (3.3), (3.4) and (3.5), we find that $\beta_{0}\left(y, r\left(n, n_{1}\right)\right)=0$ for all $y \in Y_{0}$, i.e. $\gamma\left(n, n_{1}\right) \in \Delta_{0}$. The group $\Delta_{0}$ is ergodic; it is proved in same way, as in [9, Example 1].

Theorem 3.2. The cocycle $\beta_{0} \in Z^{1}\left(Y_{0} \times \Delta, H_{0}\right)$ speciefied by (3.4) has a dense range in $H_{0}$, i.e. $r\left(\Delta, \beta_{0}\right)=H_{0}$.

The proof follows in a transparent way form Lemma 3.1.

Corollary 3.3. The action of the group $G_{0}=G \times \mathbb{R}$ associated with the pair $\left(\Delta, \beta_{0}\right)$ is isomorphic to the transitive action of $G_{0}$ on the quotient space $G_{0} / \mathbb{H}_{0}$.

The proof follows from Theorem 3.2 (see also Theorem 4.1 below).

3.2. We shall use the following notation: $\left(X_{0}, \mathscr{B}_{0}, \mu_{0}\right)$ and $\left(Y, \mathscr{F}_{,}, \nu\right)$ are the Lebesgue spaces with probability measures and $(X, \mathscr{B}, \mu)=\left(X_{0} \times Y, \mathscr{B}_{0} \times \mathscr{F}_{1}, \mu_{0} \times\right.$ $\nu) ; S$ is an ergodic automorphism of $(Y, \mathscr{F}, \nu)$ and $S_{0}=\mathbb{Z} \times S \in A u t(X, \mathscr{B}, \mu)$. Let, as previously, $\mathbb{H}_{0}$ be a closed subgroup of $G_{0}=G \times \mathbb{R}$.

Assume that for $\mu_{0}$-a.a. $x_{0} \in X_{0}$ the cocycle $\alpha\left(x_{0}\right) \in Z^{1}(Y \times[S], G)$ is defined. We shall say that in this case a field of cocycles $x_{0} \rightarrow \alpha\left(x_{0}\right)\left(x_{0} \in X_{0}\right)$ with values 
in $G$ is defined. To each field of cocycles $x_{0} \rightarrow \alpha\left(x_{0}\right)$ corresponds a map $\alpha: X \times$ $\left[S_{0}\right] \rightarrow G$

$$
\alpha\left(x_{0}, y, S_{0}^{n}\right)=\alpha\left(x_{0}\right)\left(y, S^{n}\right),
$$

which satisfies the identity for the cocycles.

Definition 3.4. A field of cocycles $x_{0} \rightarrow \alpha\left(x_{0}\right), x_{0} \in X_{0}$ is called measurable, if the cocycle $\alpha$ defined by (3.6) is measurable.

Definition 3.5. A measurable field of cocycles $x_{0} \rightarrow \alpha\left(x_{0}\right)$ has a dense range in a group $H \subset G$ (the notation $r(\{\alpha(\cdot)\})=H$ ), if for a.a. $x_{0} \in X_{0}$ the cocycle $\alpha\left(x_{0}\right)$ has a dense range in $H$.

Each measurable field of cocycles $x_{0} \rightarrow \alpha\left(x_{0}\right)$ generates also a measurable field $x_{0} \rightarrow \alpha_{0}\left(x_{0}\right)=\left(\alpha\left(x_{0}\right), \rho\right)$ of cocycles taking values in the group $H_{0}$.

Below we shall consider only measurable fields of cocycles and assume $S$ to be of type $\mathrm{II}_{1}$ or $\mathrm{III}_{\lambda}(0<\lambda \leqslant 1)$.

Lemma 3.6. The following statements are equivalent:

(i) a measurable field of cocycles $x_{0} \rightarrow \alpha_{0}\left(x_{0}\right)$ has a dense range in $H_{0}$;

(ii) for any $h_{0} \in H_{0}$, any set $A \subset X_{0} \times Y$ of positive measure and any neighborhood $V$ of the identity in $G_{0}$, there exist a measurable field of automorphisms $s_{0}=\left(x_{0} \rightarrow s\left(x_{0}\right)\right) \in\left[S_{0}\right]$ and a set $B \subset A$, such that $s_{0} B \subset A$ and $\alpha_{0}\left(x_{0}\right)\left(y, s\left(x_{0}\right)\right) \in$ $h_{0}+V$ for a.a. $\left(x_{0}, y\right) \in B$;

(iii) for any $h_{0} \in H_{0}$, any two sets $A$ and $B$ in $X_{0} \times Y$ such that $\nu\left(A\left(x_{0}\right)\right)>0$ $\Leftrightarrow \nu\left(B\left(x_{0}\right)\right)>0$ (where $A\left(x_{0}\right)$ and $B\left(x_{0}\right)$ are $x_{0}$-sections of $A$ and $B$ ) and any neighborhood $U$ of the identity in $G_{0}$, there exist a set $A^{\prime} \subset A$ of positive measure and an element $s_{0}^{\prime}=\left(x_{0} \rightarrow s^{\prime}\left(x_{0}\right)\right) \in\left[S_{0}\right]$ such that $s_{0}^{\prime} A^{\prime} \subset B$ and $\alpha_{0}\left(x_{0}\right)\left(y, s^{\prime}\left(x_{0}\right)\right) \in h_{0}+U$ for a.a. $\left(x_{0}, y\right) \in A^{\prime}$.

The proof of this lemma is similar to that of Lemma 2.1 from [5].

Choose in the group $G$ an invariant metric $d$ compatible with the topology of the group $G$.

Next lemma is formulated and proved for the case of $S$ of type III; the case of type II automorphism $S$ is to be considered similarly.

Lemma 3.7. Let a measurable field of cocycles $x_{0} \rightarrow \alpha_{0}\left(x_{0}\right)$ be such that $r\left(\left\{\alpha_{0}(\cdot)\right\}\right)=H_{0}$ and $h_{0}=\left(h_{G}, h_{R}\right) \in H_{0} . \quad$ Let $A$ and $B$ be subsets of $X=X_{0} \times Y$, such that $\nu\left(A\left(x_{0}\right)\right)=\nu\left(B\left(x_{0}\right)\right) e^{h_{R}}$, where $A\left(x_{0}\right)$ and $B\left(x_{0}\right)$ are $x_{0}$-sections of $A$ and $B$. Then, for any $\varepsilon>0$, there exists an automorphism $s_{0} \in\left[S_{0}\right]$ for which $s_{0} A=B$ 
and

$$
\alpha_{0}\left(x_{0}\right)\left(y_{0} s\left(x_{0}\right)\right) \in\left(h_{G}, h_{R}\right)+(V(\varepsilon) \times(-\varepsilon, \varepsilon)),
$$

where $s_{0}=\left(x_{0} \rightarrow s\left(x_{0}\right)\right)$ and $V(\varepsilon)=\{g \in G: d(0, g)<\varepsilon\}$.

Proof. Let us use statement (iii) of Lemma 3.6 and construct a map $t_{1} \in$ $\left[S_{0}\right]$ such that for a subset $A_{1} \subset A$ of positive measure $t_{1} A_{1}=B_{1} \subset B$ and

$$
\alpha_{0}\left(x_{0}\right)\left(y, t_{1}\left(x_{0}\right)\right) \in h_{0}+V_{0}(\varepsilon),\left(x_{0}, y\right) \in A_{1},
$$

where $t_{1}=\left(x_{0} \rightarrow t_{1}\left(x_{0}\right)\right)$ and $V_{0}(\varepsilon)=V(\varepsilon) \times(-\varepsilon, \varepsilon)$. Choose here $A_{1}$ so that

$$
\mu\left(t_{1} A_{1}\right) \leqslant e^{h_{R}} \mu\left(A_{1}\right) \text {. }
$$

Consider now the set of pairs $\left(A_{1}, t_{1}\right)$ satisfying (3.8) and (3.9) and define on this set a partial order relation, assuming $\left(A_{1}^{\prime}, t_{1}^{\prime}\right)<\left(A_{1}^{\prime \prime}, t_{1}^{\prime \prime}\right)$ if $A_{1}^{\prime} \subset A_{1}^{\prime \prime}$ and $t_{1}^{\prime}=t_{1}^{\prime \prime}$ on $A_{1}^{\prime}$. By Zorn lemma, there exists a maximal pair $\left(A_{0}, t_{0}\right)$ with respect to such the order relation. Therefore, we readily conclude that $A_{0}=A$ by mod 0 and the automorphism $t_{0}=\left(x_{0} \rightarrow t\left(x_{0}\right)\right)$ satisfies the relation

$$
\alpha_{0}\left(x_{0}\right)\left(y, t\left(x_{0}\right)\right) \in h_{0}+V_{0}(\varepsilon)
$$

for a.a. $\left(x_{0}, y\right) \in A$. Besides, $\mu\left(t_{0} A\right) \leqslant \mu(B)$.

For the symmetry reasons, there exists an automorphism $w_{0}=\left(x_{0} \rightarrow w\left(x_{0}\right)\right) \in$ $\left[S_{0}\right]$ such that $w_{0} B \subset A$ and

$$
\alpha_{0}\left(x_{0}\right)\left(y, w\left(x_{0}\right)\right) \in-h_{G}+V_{0}(\varepsilon)
$$

for a.a. $\left(x_{0}, y\right) \in B$. From the maps $t_{0}$ and $w_{0}$, as in [9] we shall construct the Bernstein map $s_{0}=\left(x_{0} \rightarrow s\left(x_{0}\right)\right) \in\left[S_{0}\right]$ which is the one-to-one map from $A$ onto $B$ :

$$
s_{0}\left(x_{0}, y\right)=\left\{\begin{array}{l}
t_{0}\left(x_{0}, y\right),\left(x_{0}, y\right) \in \bigcup_{i=0}^{\infty}\left[\left(w_{0} t_{0}\right)^{i} A-w_{0}\left(t_{0} w_{0}\right)^{i} B\right] \cup \bigcap_{i=0}^{\infty}\left(w_{0} t_{0}\right)^{i} A \\
\left.w_{0}^{-1}\left(x_{0}, y\right),\left(x_{0}, y\right) \in \bigcup_{i=0}^{\infty}\left(w_{0}\left(t_{0} w_{0}\right)^{i} B-\left(w_{0}, t_{0}\right)\right)^{i+1} A\right) .
\end{array}\right.
$$

From (3.10) and (3.11), follows (3.7).

Further it will be convenient to believe the cocycle $\alpha_{0}$ to take values in the countable subgroup $H_{0}^{\prime}$ dense in $H_{0}$ (see Subsection 3.1). This assumption is not restrictive [6].

Lemma 3.8. Let $A$ be a measurable subset of positive measure in $X_{0} \times Y$ and $\left\{h_{0}(i)\right\}_{i=1}^{N}$ a set of elements of the group $H_{0}^{\prime}$, where $h_{0}(i)=\left(h_{G}(i), h_{R}(i)\right)$ and let the 
range of a measurable field of cocycles $x_{0} \rightarrow \alpha_{0}\left(x_{0}\right)$ be the group $H_{0}$. Assume that $\xi=(A,[0, N-1], B(i), r(\cdot, \cdot))$ is an $S_{0}$-array of $A$ such that $\mu(B(i))=\exp \left(h_{R}(i)\right)$ $\mu(B(0))$ and for certain $\varepsilon>0$ and a.a. $\left(x_{0}, y\right) \in B(0)$

$$
\alpha_{0}\left(x_{0}\right)(y, r(i, 0)) \in h_{0}(i)+V_{0}(\varepsilon), \quad i \in[1, N-1],
$$

where $V_{0}(\varepsilon)=V(\varepsilon) \times(-\varepsilon, \varepsilon)$. Then, there exists a measurable function $f_{0}: A \rightarrow H_{0}^{\prime}$ such that $f_{0}\left(x_{0}, y\right)=0$ for a.a. $\left(x_{0}, y\right) \in B(0)$ and

$$
f_{0}\left(x_{0}, y\right) \in V_{0}(\varepsilon), \quad\left(x_{0}, y\right) \in A
$$

and for the measurable field of cocycles

$$
\beta_{0}\left(x_{0}\right)(y, s)=f_{0}\left(x_{0}, s y\right)+\alpha_{0}\left(x_{0}\right)(y, s)-f_{0}\left(x_{0}, y\right), \quad s \in[S]
$$

the following relations are true for a.a. $\left(x_{0}, y\right) \in B(0)$ :

$$
\beta_{0}\left(x_{0}\right)(y, r(i, 0))=h_{0}(i), \quad i=1,2, \cdots, N-1 .
$$

Proof. Put for $i=1,2, \cdots, N-1$ the following

$$
f_{0}\left(x_{0}, y\right)=\left\{\begin{array}{l}
0 \\
h_{0}(i)-\alpha_{0}\left(r(i, 0)^{-1}\left(x_{0}, y\right), r(i, 0)\right),\left(x_{0}, y\right) \in B(i),
\end{array}\right.
$$

where $\alpha_{0}$ satisfies (3.6). Simple check shows that (3.13) and (3.15) follow from (3.12), (3.14) and (3.16).

In other words, Lemma 3.8 states that the cocycle $\alpha$ may be replaced by the $S_{0}$-cohomologous cocycle $\beta$ and the measure $\nu$ by the measure $\nu^{\prime}$ equivalent to it, so that on the elements of the $S_{0}$-array $\xi$ the cocycle $\beta_{0}$ should have constant values, the Radon-Nikodym cocycle in particular also becoming constant on such elements. In this case, the function $f_{0}$ performing the cohomologous replacement takes values in the prescribed neighborhood of the identity in $H_{\mathbf{0}}$.

3.3. Before starting to prove the uniqueness theorem for fields of cocycles, let us consider the uniqueness theorem for individual cocycles with a dense range in the prescribed group $H_{0} \subset G_{0}$. The proof method of this theorem will then be extended, in a transparent way, to the case of measurable fields of cocycles.

Let $\Gamma$ be an ergodic a.f. group of automorphisms of $(X, \mathscr{B}, \mu), \mu(X)=1$ and an automorphism $T \in \operatorname{Aut}(X, \mathscr{B}, \mu)$ be such that $[\Gamma]=[T]$; let $H_{0}$ be a closed subgroup of $G_{0}$. Assume the cocycle $\alpha_{0}=(\alpha, \rho)$ to take values in $H_{0}$ and $H_{0}=r(\Gamma, \alpha)$. As has been said, according to whether the group $H_{\boldsymbol{R}}$ (where $H_{0}$ 
$\left.=\left(H_{G}, H_{R}\right)\right)$ is trivial, or discrete of the form $\mathbb{Z} \log \lambda$, or dense in $\mathbb{R}$, the group $\Gamma$ is of types $\mathbb{I I}_{1}, \mathbb{I I}_{\lambda}(0<\lambda<1)$, or $\mathbb{I I I}_{1}$. The case of $\Gamma$ of type $\mathbb{I I}$ was considered in [6].

Here we shall prove the following uniqueness theorem for the group $T$ of automorphisms of type $\mathbb{I I I}$.

Theorem 3.9. Let the groups of automorphisms $\Gamma_{i} \subset \operatorname{Aut}\left(X_{i}, \mathscr{B}_{i}, \mu_{i}\right)$ and the cocycles $\alpha_{0}^{i}, i=1,2$ satisfy the conditions: $\alpha_{0}^{i} \in Z^{1}\left(X_{i} \times \Gamma_{i}, H_{0}\right), r\left(\Gamma_{i}, \alpha_{0}^{i}\right)=H_{0}$, i.e. let the associated actions $W_{\left(\Gamma_{i}, \alpha_{0}^{i}\right)}\left(G_{0}\right)$ coincide with the transitive action of the group $G_{0}$ on $G_{0} / H_{0}$. Then, the pairs $\left(\Gamma_{1}, \alpha_{0}^{1}\right)$ and $\left(\Gamma_{2}, \alpha_{0}^{2}\right)$ are weakly equivalent.

This theorem will be proved in several steps. Note now that Lemmas 3.6, 3.7, and 3.8 remain valid, if the field of cocycles in their formulations is replaced by one fixed cocycle.

Lemma 3.10. Let $\xi=\left(X,[1, N], A\left({ }^{\circ}\right), \gamma\left({ }^{\circ},{ }^{\circ}\right)\right)$ be a $\Gamma$-array on the set $X$, such that for $\varepsilon>0$ and a set $D \subset X$ of positive measure, the following is true:

$$
\begin{aligned}
& \mu(\{x \in X: T x \in \mathcal{G}(\xi) x\})>1-\varepsilon, \\
& \mu\left(D \Delta D_{1}\right)<\varepsilon,
\end{aligned}
$$

where $D_{1} \in \mathscr{L}(\xi)$ and $\Gamma, T$ and $\alpha_{0}$ satisfy the conditions enumerated above. Then, there exists a $\Gamma$-array $\xi_{1}$ such that

$$
\begin{aligned}
& \mu\left(\left\{x \in X: T x \in \mathcal{G}\left(\xi_{\S}\right) x\right\}\right)>1-2 \varepsilon, \\
& \mu\left(D \Delta D_{1}^{\prime}\right)<2 \varepsilon,
\end{aligned}
$$

where $D_{1}^{\prime} \in \mathscr{Q}\left(\xi_{1}\right)$. Moreover, the cocycle $\alpha_{0}$ is cohomologous to a cocycle $\alpha_{0}^{\prime}$ which takes constant values on all elements of $\xi_{1}$, a function $f_{0}$ specifying the cohomologous replacement takes values in the neighborhood $V_{0}(2 \varepsilon)=V(2 \varepsilon) \times(-2 \varepsilon$, $2 \varepsilon$ ) of the identity in $G_{0}$.

Proof. Since $\alpha_{0}$ takes values in the countable subgroup $H_{0}^{\prime} \subset H_{0}$, then the functions $f^{i}(x)=\alpha_{0}(x, \gamma(i, 1)), i=1,2, \cdots, N$, where $x \in A(1)$, are piecewise constant. Let $\left\{E_{\omega}: \omega \in \Omega\right\}$ be the partition of $A(1)$ into the sets on which $f^{i}(x)$, $i=1,2, \cdots, N$ are constant. The set $\Omega$ is finite or countable (we shall naturally assume it countable). Let $\Omega^{\prime}$ be a finite subset of $\Omega$, such that

$$
\mu\left(\bigcup_{\omega \in \Omega^{\prime}} \bigcup_{i=1}^{\pi} \gamma(i, 1) E_{\omega}\right)>1-\frac{\varepsilon}{2} \text {. }
$$

Let us believe for definiteness that $f^{i}(x)=\left(h_{\omega}^{i}, a_{\omega}^{i}\right)$ for $x \in E_{\omega}, \omega \in \Omega, i \in[1, N]$. Then, for the same values of $\omega, i$ 


$$
\mu\left(\gamma(i, 1) E_{\omega}\right)=e^{a_{\omega}^{i} \mu}\left(E_{\omega}\right) .
$$

Put $q_{\omega}=\mu\left(E_{\omega}\right), \omega \in \Omega$. The condition (3.19) then is

$$
\sum_{\omega \in \Omega^{\prime}} \sum_{i=1}^{N} e_{\omega \omega}^{a_{\omega}^{i}} q_{\omega}>1-\frac{\varepsilon}{2} .
$$

From the given numbers $\varepsilon>0$ and $\left\{q_{\omega}\right\}_{\omega \in \Omega^{\prime}}$, we can find the numbers $q>0$ and $n_{\omega} \in N$ such that

$$
\begin{aligned}
& n_{\omega} q \leqslant q_{\omega}<\left(n_{\omega}+1\right) q, \\
& \sum_{i=1}^{N} \sum_{\omega \in \Omega^{\prime}} e^{a_{\omega}^{i}}\left(q_{\omega}-n_{\omega} q\right)<\frac{\varepsilon}{2},
\end{aligned}
$$

Let $F_{\omega} \subset E_{\omega}, \mu\left(F_{\omega}\right)=q, \omega \in \Omega^{\prime}$. Inequalities (3.21) show that there exists a collection of sets $\left\{F_{\omega}(k)\right\}_{k=1}^{n_{\omega}}$ from $E_{\omega}$ such that $F_{\omega}(1)=F_{\omega}, F_{\omega}\left(k_{1}\right) \cap F_{\omega}\left(k_{2}\right)=\varnothing\left(k_{1} \neq\right.$ $\left.k_{2}\right), \mu\left(F_{\omega}(k)\right)=q$ for $k=1,2, \cdots, n_{\omega}, \omega \in \Omega^{\prime}$. Put

$$
\begin{gathered}
A^{\prime}(1)=\bigcup_{\omega \in \Omega^{\prime}} \bigcup_{k=1}^{n_{\omega}} F_{\omega}(k), \\
A^{\prime \prime}(1)=\left(\bigcup_{\omega \in \Omega \backslash \Omega^{\prime}} E_{\omega}\right) \cup\left(\bigcup_{\omega \in \Omega^{\prime}}\left(E_{\omega}-\bigcup_{k=1}^{n_{\omega}} F_{\omega}(k)\right)\right) .
\end{gathered}
$$

Then, $A(1)=A^{\prime}(1) \cup A^{\prime \prime}(1)$ and $A^{\prime}(1) \cap A^{\prime \prime}(1)=\varnothing$. From (3.19), (3.20) and (3.22), it follows that

$$
\mu\left(\bigcup_{j=1}^{N} r(j, 1) A^{\prime \prime}(1)\right)<\varepsilon .
$$

Let us number the sets $F_{\omega}(k)$ successively: $F(1) ; F(2), \cdots, F(M)$, where $M=$ $\sum_{\omega \in \Omega^{\prime}} n_{\omega}$. Also, denote the functions $f^{i}(x)$ on $F(j)$ in a different way, putting $f^{i}(x)=\left(g_{j}^{i}, b_{j}^{i}\right)$ for $x \in F(j)$. The collection $\left\{\left(g_{j}^{i}, b_{i}^{j}\right): i=1,2, \cdots, N ; j=1,2, \cdots\right.$, $M\}$ obviously coincides with the collection $\left\{\left(h_{\omega}^{i}, a_{\omega}^{i}\right): i=1,2, \cdots, N ; \omega \in \Omega^{\prime}\right\}$. Therefore, (3.20) leads to $\mu(r(i, 1) F(j))=q \exp \left(b_{j}^{i}\right)$.

By applying Lemma 3.7, consider over the partition $\left(A^{\prime}(1),[1, M], F(\cdot)\right)$ a $\Gamma$-array $\eta_{1}^{\prime}$, whose automorphisms $\delta(\cdot, \cdot)$ have the property

$$
\alpha_{0}(x, \delta(j, 1)) \in V_{0}(\varepsilon), \quad j=1,2, \cdots, M
$$

for a.a. $x \in F(1)$. Denote by $\eta^{\prime}$ a refinement of the array $\xi$ by the array $\eta_{1}^{\prime}$ defined on the set $A=\cup_{i=1}^{N} \gamma(i, 1) A^{\prime}(1)$. The array $\eta^{\prime}$ consists of the sets $r(i$, 1) $F(j)$ with the measure $e^{b^{i}}{ }_{j}, i=1,2, \cdots, N ; j=1,2, \cdots, M$ and the group $\mathcal{G}\left(\eta^{\prime}\right)$ consists of the automorphisms $t^{\prime}(\cdot, \cdot)$ defined by relations of the form $r(i, 1)$ $\delta(\cdot, \cdot) r(i, 1)^{-1}, i, j \in 1,2, \cdots, M$. For convenience, let us number again the sets of $\eta^{\prime}$ successively: $\eta^{\prime}=\left(A,[1, M N], C^{\prime}(\cdot), t^{\prime}(\cdot, \cdot)\right)$. Let us find out what the values are that the cocycle $\alpha_{0}$ takes on elements of $\xi^{\prime}$. Let for definiteness, 
$C^{\prime}(1)=F(1)$. If $C^{\prime}(n) \subset A^{\prime}(1)$, then, in view of $(3.24) \alpha_{0}\left(x, t^{\prime}(n, 1)\right) \in V_{0}(\varepsilon)$ for $x \in C^{\prime}(1)$; if $C^{\prime}(n) \subset r(i, 1) A^{\prime}(1)$, then for $x \in C^{\prime}(1)$

$$
\alpha_{0}\left(x, t^{\prime}(n, 1)\right)=\alpha_{0}(x, \gamma(i, 1) \delta(k, 1)) \in\left(g_{k}^{i}, b_{k}^{i}\right)+V_{0}(\varepsilon),
$$

where $k$ is specified by the equality $F(k)=r(i, 1)^{-1} C^{\prime}(n)$. Therefore, put $(g(n)$, $b(n))=(0,0)$ for $C^{\prime}(n) \subset A^{\prime}(n)$ and $(g(n), b(n))=\left(g_{k}^{i}, b_{k}^{i}\right)$ for $C^{\prime}(n)=r(i, 1) F(k)$. Thus, for a.a. $x \in C(1)$

$$
\alpha_{0}\left(x, t^{\prime}(n, 1)\right) \in(g(n), b(n))+V_{0}(\varepsilon), \quad n=1,2, \cdots, N M .
$$

Then, $\mu\left(C^{\prime}(n)\right)=e^{b(n)} q=r_{n}$.

Using (3.23), calculate the measure of the set $B=\bigcup_{i=1}^{N} \gamma(i, 1) A^{\prime \prime}(1)$ :

$$
\mu(B)=1-\sum_{n=1}^{N M} e^{b(n)} q=1-\sum_{n=1}^{N M} r_{n}<\varepsilon
$$

Subdivide $B$ into the nonintersecting subsets $C^{\prime \prime}(n), n \in[1, N M]$ such that

$$
\mu\left(C^{\prime \prime}(n)\right)=\mu\left(C^{\prime}(n)\right) \mu(B) \mu(A)^{-1} \text { 。 }
$$

Since $b(n)=\log r_{n} r_{1}^{-1}$, then for $r_{n}^{\prime}=\mu\left(C^{\prime \prime}(n)\right)$ it follows from (3.26) that $b(n)=$ $\log r_{n}^{\prime}\left(r_{n}^{\prime}\right)^{-1}$, i.e. $r_{n} r_{1}^{-1}=r_{n}^{\prime}\left(r_{1}^{\prime}\right)^{-1}$. Since $(g(n), b(n)) \in H_{0}^{\prime}$, then, by Lemma 3.7, over the partition $\left(B,[1, N M], C^{\prime \prime}(\cdot)\right)$ a $\Gamma$-array $\eta^{\prime \prime}$ can be defined having the automorphisms $t^{\prime \prime}\left({ }^{\circ}\right)$ such that for a.a. $x \in C^{\prime \prime}(1)$

$$
\alpha_{0}\left(x, t^{\prime \prime}(n, 1)\right) \in(g(n), b(n))+V_{0}(\varepsilon), \quad n \in[1, N M] .
$$

Construct the $\Gamma$-array $\xi_{1}=(X,[1, N M], C(\cdot), t(\cdot, \cdot))$ from the arrays $\eta^{\prime}$ and $\eta^{\prime \prime}$, putting

$$
\begin{aligned}
C(n) & =C^{\prime}(n) \cup C^{\prime \prime}(n), \\
t(n, 1) x & = \begin{cases}t^{\prime}(n, 1) x, & x \in C^{\prime}(1) \\
t^{\prime \prime}(n, 1) x, & x \in C^{\prime \prime}(1), \quad n=1,2, \cdots, N M .\end{cases}
\end{aligned}
$$

Then, it follows from (3.25) and (3.27) that

$$
\alpha_{0}(x, t(n, 1)) \in(g(n), b(n))+V_{0}(\varepsilon), \quad n=1,2, \cdots, N M .
$$

Apply Lemma 3.8 to the array $\xi_{1}$. Then, there exists a measurable function $f_{0}(x)$ taking values in $V_{0}(\varepsilon)$ and such that the cocycle

$$
\alpha_{0}^{\prime}(x, t)=f_{0}(t x)+\alpha_{0}(x, t)-f_{0}(x), \quad t \in[T]
$$

has the property

$$
\alpha_{0}^{\prime}(x, t(n, 1))=(g(n), b(n)), \quad n=1,2, \cdots, N M,
$$


for a.a. $x \in C(1)$. It follows from (3.17) and (3.23) that the array $\xi_{1}$ satisfies (3.18). Equalities (3.30) show that the cocycle $\alpha_{0}^{\prime}$ cohomologic to $\alpha_{0}$ takes constant values on elements of $\xi_{1}$.

To conclude the proof of the lemma, let us estimate the change of the measure of $X$ as a result of replacement of $\mu$ by $\mu^{\prime}$ by formula (3.29) by means of the function $f_{R}(x)$, where $f_{0}(x)=\left(f_{G}(x), f_{R}(x)\right)$. It follows from (3.29) that

$$
\left.e^{-\varepsilon}<\mu^{\prime}(X)=\int_{X} \exp \left(f_{R}(x)\right) d \mu(x)\right)<e^{\varepsilon} .
$$

Normalize the measure $\mu^{\prime}$, putting $\mu_{1}^{\prime}(E)=\mu^{\prime}(E) \mu^{\prime}(X)^{-1}$ for $E \in \mathscr{B}$. This means that $f_{R}(x)$ is replaced by the function $f_{R}^{\prime}(x)$ such that $\exp \left(f_{R}^{\prime}(x)\right)=\mu^{\prime}(X)^{-1}$ $\exp \left(f_{R}(x)\right)$. It follows from (3.31) that $f_{R}^{\prime}(x) \in(-2 \varepsilon, 2 \varepsilon)$.

Lemma 3.11. Let $(X, \mathscr{B}, \mu), \Gamma, T, \alpha_{0}, H_{0}$ and $H_{0}^{\prime}$ be as above. Then, there exists a sequence $\left\{\xi_{n}\right\}_{n=1}^{\infty}$ of $\Gamma$-arrays and a cocycle $\alpha_{0}^{\prime}$ cohomologous to the cocycle $\alpha_{0}$, both such that

(i) $\xi_{n+1}$ is the refinement of $\xi_{n}, n \in N$;

(ii) $\left\{T_{X}^{m}: m \in Z\right\}=\cup_{n=1}^{\infty} \mathcal{G}\left(\xi_{n}\right) x$ for a.a. $x \in X$;

(iii) $\mathscr{B}=\sigma\left(\cup_{n=1}^{\infty} \mathcal{Q}\left(\xi_{n}\right)\right)$;

(iv) on any element of $\xi_{n}, n \in \boldsymbol{N}$ the cocycle $\alpha_{0}^{\prime}$ takes a constant value.

Proof. Let a sequence of positive numbers $\left\{\varepsilon_{n}\right\}_{n=1}^{\infty}$ monotonically converges to zero and $\sum_{n=1}^{\infty} \varepsilon_{n}<\infty$. Let $\left\{D_{n}\right\}_{n=1}^{\infty}$ be a dense sequence of sets in $\mathscr{B}$, whose every element occurs in it an infinite number of times. Apply Lemma 3.10 and construct a $\Gamma$-array $\xi_{1}=\left(X,\left[1, N_{1}\right], A_{1}(\cdot), \gamma_{1}(\cdot, \cdot)\right)$ and a cocycle $\alpha_{0}^{(1)}$ cohomologous to $\alpha_{0}$, such that the group $\mathcal{G}\left(\xi_{1}\right)$ approximates the orbits of $T$ accurate to $\varepsilon_{1}$ and $\mathscr{Q}\left(\xi_{1}\right)$ approximates the set $D_{1}$ also accurate to $\varepsilon_{1}$ (i.e. inequalities similar to (3.17) are true). The cocycle $\alpha_{0}^{(1)}$ takes constant values on elements of $\xi_{1}$ and is obtained from the cocycle $\alpha_{0}$ by the cohomologous transition defined by a transfer function $f_{0}^{(1)}(x)$. This function has the properties: $f_{0}^{(1)}(x)$ $\in V_{0}(\varepsilon)$ and $f_{0}^{(1)}(x)=0$ for $x \in A_{1}(1)$. Then, construct on $A_{1}(1)$ a $\Gamma$-array $\xi_{1}^{\prime}$ such that the array $\xi_{2}=\xi_{1} \times \xi_{1}^{\prime}$ approximates the orbits of $T$ and the set $D_{1}$ accurate to $\varepsilon_{2}$. Here, if $\xi_{1}^{\prime}=\left(A_{1}(1),\left[1, N_{1}^{\prime}\right], A_{1}^{\prime}(\cdot), \gamma_{1}^{\prime}(\cdot, \cdot)\right)$, then on the set $A_{1}^{\prime}(1)$ one can define the function $\bar{f}_{0}^{(1)}(x)$ such that $\bar{f}_{0}^{(1)}(x)=0$ on $A_{1}^{\prime}(1)$ and $\bar{f}_{0}^{(1)}(x) \in V_{0}\left(\varepsilon_{2}\right)$. Denote by $f_{0}^{(2)}(x)$ the $\mathcal{G}\left(\xi_{1}\right)$-invariant function obtained from $\bar{f}_{0}^{(1)}(x)$ by shifts by $r_{1}(i, 1), i=1,2, \cdots, N_{1}$. According to Lemma $3.10 \bar{f}_{0}^{(1)}(x)$ and $\xi_{1}^{\prime}$ can be chosen to be such that the cocycle $\alpha_{0}^{(2)}(x, t)=f_{0}^{(2)}(t x)+\alpha_{0}^{(1)}(x, t)-f_{0}^{(2)}(x)$ takes constant values on elements of the array $\xi_{2}, t \in[T]$.

Continuing the procedure let us construct the sequence of the arrays $\left\{\xi_{n}\right\}_{n=1}^{\infty}$ 
satisfying conditions (i)-(iii) of the present lemma and also the sequence of the functions $\left\{f_{0}^{(n)}(x)\right\}_{n=1}^{\infty}$ such that the series $\sum_{n=1}^{\infty} f_{0}^{(n)}(x)$ converges to a function $f_{0}(x)$. Then, according to the choice of $f_{0}^{(n)}(x)$, the cocycle $\alpha_{0}^{\prime}(x, t)=f_{0}(t x)+$ $\alpha_{0}(x, t)-f_{0}(x), t \in[T]=[\Gamma]$ takes the constant values on all elements of the array $\xi_{n}, n \in \mathbb{N}$.

Remark 3.12. It follows from the construction of the function $f_{0}$ that, generally speaking, $f_{0} \notin H_{0}^{\prime}$. However, the cocycle $\alpha_{0}^{\prime}$ takes, as $\alpha_{0}$ does, its values in the group $H_{0}^{\prime}$ because the functions $f_{0}^{(n)}(x)$ are invariant with respect to automorphisms from the group $\mathcal{G}\left(\xi_{\S}\right), i=1,2, \cdots, n ; n \in \mathbb{N}$.

Proof of Theorem 3.9. Recall that the cocycles $\alpha_{0}^{i}, i=1,2$ take the values in the group $H_{0}^{\prime} \subset H_{0}$. Let $\left\{\varepsilon_{n}\right\}_{n=1}^{\infty}$ and $\left\{D_{n}\right\}_{n=1}^{\infty}$ be sequences the same as in proof of Lemma 3.11. Define for $\varepsilon_{1}, D_{1}$ and $\alpha_{0}^{1}$ a $\Gamma_{1}$-array $\xi_{1}$ and a $\Gamma_{1}$ cohomologous cocycle $\bar{\alpha}_{0}^{1}(1)$ satisfying Lemma 3.10. Then, $\bar{\alpha}_{0}^{1}(1)$ takes constant values on the elements of $\xi_{1}$, and $\xi_{1}$ approximates $T_{1}\left(\left[T_{1}\right]=\left[\Gamma_{1}\right]\right)$ and $D_{1}$ accurate to $\varepsilon_{1}$. Using Lemmas 3.6,3.7 and 3.8, consiruct for $\alpha_{0}^{2}$ a $\Gamma_{2}$-array $\eta_{1}$ with the same number of sets as in the array $\xi_{1}$ and define a cocycle $\bar{\alpha}_{0}^{2}(1), \Gamma_{2^{-}}$ cohomologous to $\alpha_{0}^{2}$, both such that on sets with the same indices the values of $\bar{\alpha}_{0}^{1}(1)$ and $\bar{\alpha}_{0}^{2}(1)$ coincide. That this construction is possible follows from the facts that $r\left(\Gamma_{1}, \alpha_{0}^{1}\right)=r\left(\Gamma_{2}, \alpha_{0}^{2}\right)$ and the groups $\Gamma_{1}$ and $\Gamma_{2}$ are weakly equivalent. Then, define a refinement $\eta_{2}$ of the array $\eta_{1}$ so that the approximations of $T_{2}\left(\left[T_{2}\right]=\left[\Gamma_{2}\right]\right)$ and of $D_{1}$ should have the accuracy to $\varepsilon_{2}$. Next, construct a cocycle $\bar{\alpha}_{0}^{2}(2)$ cohomologous to $\bar{\alpha}_{0}^{2}(1)$, so that the values of $\bar{\alpha}_{0}^{2}(2)$ should be constant on elements of $\eta_{2}$. From the proof of Lemma 3.11, it follows that $\bar{\alpha}_{0}^{2}(2)$ takes constant values on elements of $\eta_{1}$ as well. Returning to the $\Gamma_{1}$-cocycle $\bar{\alpha}_{0}^{1}(1)$ and the $\Gamma_{1}$-array $\xi_{1}$, define a refinement $\xi_{2}$ of $\xi_{1}$ and a cocycle $\bar{\alpha}_{0}^{1}(2)$, so that the array $\xi_{2}$ should have as many sets as $\eta_{2}$ has and that on sets with the same indices the values of $\bar{\alpha}_{0}^{1}(2)\left(\Gamma_{1}\right.$-cohomologous to $\left.\bar{\alpha}_{0}^{1}(1)\right)$ should coincide with the values of $\bar{\alpha}_{0}^{2}(2)$. In transition to cohomologous cocycles, as in Lemma 3.11, the functions $f_{0}^{1}(1), f_{0}^{1}(2)$ and $f_{0}^{2}(1), f_{0}^{2}(2)$ are constructed that define cohomologous equivalence of cocycles and are such that $f_{0}^{i}(j) \in V_{0}\left(\varepsilon_{j}\right)$, $i, j=1,2$.

By repeating the above procedure a countable number of times, we obtain two sequences of the arrays $\left\{\xi_{n}\right\}_{n=1}^{\infty},\left\{\eta_{n}\right\}_{n=1}^{\infty}$ two sequences of the cocycles $\left\{\bar{\alpha}_{0}^{1}(i)\right\}_{i=1}^{\infty},\left\{\alpha_{0}^{2}(i)\right\}_{i=1}^{\infty}$ and two sequences of the functions $\left\{f_{0}^{1}(k)\right\}_{k=1}^{\infty},\left\{f_{0}^{2}(k)\right\}_{k=1}^{\infty}$ corresponding to the groups $\Gamma_{1}$ and $\Gamma_{2}$, respectively. The arrays $\xi_{n}$ and $\eta_{n}$ satisfy conditions (i)-(iv) of Lemma 3.11 , and the functions $f_{0}^{j}(k)$ and the co- 
cycles $\alpha_{0}^{j}(k), j=1,2$ are related as

$$
\bar{\alpha}_{0}^{j}(k)(x, t)=\sum_{i=1}^{k} f_{0}^{j}(i)(t x)+\alpha_{0}^{j}(x, t)-\sum_{i=1}^{k} f_{0}^{j}(i)(x), t \in\left[\Gamma_{j}\right]
$$

and

$$
f_{0}^{j}(i)(x) \in V_{0}\left(\varepsilon_{i}\right) .
$$

Besides, the cocycles $\bar{\alpha}_{0}^{1}(k)$ and $\bar{\alpha}_{0}^{2}(k)$ have equal values on elements of $\xi_{k}$ and $\eta_{k}$ that have the same indices. Since $\sum_{n=1}^{\infty} \varepsilon_{n}<\infty$, then in view of (3.33), we can assume $f_{0}^{j}(x)=\sum_{i=1}^{\infty} f_{0}^{j}(i)(x), j=1,2$. Then, it follows from (3.32) that for $j=$ 1,2 the cocycles $\bar{\alpha}_{0}^{j}(x, t)=f_{0}^{j}(t x)+\alpha_{0}^{j}(x, t)-f_{0}^{j}(x), t \in\left[\Gamma_{j}\right]$ are defined and their values are constant on all elements of the arrays $\xi_{n}$ and $\eta_{n} n \in N$. Besides, if take elements of $\xi_{n}$ and $\eta_{n}$ having the same indices, then the cocycles $\bar{\alpha}_{0}^{1}$ and $\bar{\alpha}_{0}^{2}$ take on them equal values (see Remark 3.12).

The above enumerated properties enable conclusion (as in [1]) that there exists an automorphism $\theta$ for which $\theta\left[\Gamma_{2}\right] \theta^{-1}=\left[\Gamma_{1}\right]$ and $\bar{\alpha}_{0}^{1}\left(\theta x, \theta t \theta^{-1}\right)=\bar{\alpha}_{0}^{2}(x, t)$, $t \in\left[\Gamma_{2}\right]$. Putting it otherwise, the pairs $\left(\Gamma_{1}, \alpha_{0}^{1}\right)$ and $\left(\Gamma_{2}, \alpha_{0}^{2}\right)$ are weakly equivalent.

3.4. Let us come back to considering the fields of cocycles which we began in Subsection 3.2. To prove Theorem 3.9 and Lemmas 3.10 and 3.11, we used the results of the said subsection (Lemmas 3.6, 3.7 and 3.8) that are true for the fields of cocycles. Therefore, the proofs of the results of Subsection 3.3 formulated for individual cocycles can be extended without changes to the case of the fields of cocycles. Then, we obtain validity of

Theorem 3.13. Let $\left(X_{0}, \mathcal{B}_{0}, \mu_{0}\right)$ and $(Y, \mathscr{F}, \nu)$ be Lebesgue spaces, $S$ an ergodic automorphism of $(Y, \mathscr{F}, \nu), x_{0} \rightarrow \alpha_{0}^{i}\left(x_{0}\right), i=1,2$ ineasurables fields of cocycles with values in a group $H_{0}$ such that $r\left(\left\{\alpha_{0}^{1}(\cdot)\right\}\right)=r\left(\left\{\alpha_{0}^{2}(\cdot)\right\}\right)=H_{0}$. Then, there exists a measurable field of automorphism $P_{0}=\left(x_{0} \rightarrow P\left(x_{0}\right)\right)$ such that $P\left(x_{0}\right) \in$ $N[S]$ and the cocycle $P_{0} \circ \alpha_{0}^{1}$ is $S_{0}$-cohomologous to the cocycle $\alpha_{0}^{2}$, where $S_{0}=\mathbb{1} \times$ $S$. In other words, $\left(S_{0}, \alpha_{0}^{1}\right)$ and $\left(S_{0}, \alpha_{0}^{2}\right)$ are weakly equivalent.

In Subsection 3.1, the cocycle $\beta_{0}$ with values in $H_{0}$ and a dense range in $H_{0}$ was constructed. By $\beta_{0}$ we shall also denote the constant field of cocycles, each equal to $\beta_{0}$.

Corollary 3.14. Let the conditions of Theorem 3.13 be fulfilled and $r\left(\left\{\alpha_{0}(\cdot)\right\}\right)=H_{0}$. Then, there exists a measurable field of automorphisms $P_{0}=$ $\left(x \rightarrow P\left(x_{0}\right)\right) \in N\left[S_{0}\right]$ such that the cocycle $P_{0} \circ \alpha_{0}$ is $S_{0}$-cohomologous to the constant 
field of cocycles $\beta_{0}$.

Corollary 3.15. Let a measurable field of cocycles $x_{0} \rightarrow \alpha_{0}\left(x_{0}\right)\left(x_{0} \in X_{0}\right)$ has a dense range in $H_{0}$ and takes values in $H_{0}$. Then, it can be replaced by a cohomologous field of cocycles $x_{0} \rightarrow \alpha_{0}^{\prime}\left(x_{0}\right)$ (thereby, the measure on $Y$ will also be replaced by an equivalent one), so that the group $\left\{s \in[S]: \alpha_{0}^{\prime}\left(x_{0}\right)(y, s)=0, y \in Y\right\}$ will be ergodic for a. $a_{0} x_{0} \in X_{0}$.

Remark 3.16. Analysis of Theorems 3.9 and 3.13 shows that they remain true also when the cocycle $\alpha_{0}=(\alpha, \rho)$ takes values in a closed normal amenable subgroup $H_{0}$ of $G \times \mathbb{R}$ so that the group $H_{0}$ is dense range of $\alpha_{0}$ and $G$ is an arbitrary amenable 1.c.s. group.

\section{§4. Transitive Associated Actions}

4.1. Let $\Gamma$ be an arbitrary countable ergodic group of automorphisms of $(X, \mathscr{B}, \mu)$; $G$ a nonabelian 1.c.s. group and $\alpha \in Z^{1}(X \times \Gamma, G)$.

Theorem 4.1. Let the associated action $W_{(\Gamma, \alpha)}(G)$ for a pair $(\Gamma, \alpha)$ be isomorphic to the transitive action of the group $G$ on a quotient space $G / H$, where $H$ is a proper closed subgroup of $G$. Then, the cocycle $\alpha$ is $\Gamma$-cohomologous to a cocycle $\beta$ taking all its values in $H$. If $G$ is abelian, then $r(\Gamma, \alpha)=r(\Gamma, \beta)=H$.

Proof. As in Section 2, we shall consider the skew product $\Gamma(\alpha) \subset$ Aut $\left(X \times G, \mu \times \chi_{G}\right)$ and the action $V$ of $G$ which are defined by (2.1) and (2.2). Let $\xi$ be a measurable hull of partition into orbits of the group $\Gamma(\alpha)$. By the condition of the theorem, the quotient space $(\Omega, \nu)=\left((X \times G) / \xi,\left(\mu \times \chi_{G}\right) / \xi\right)$ is isomorphic to the quotient space $G / H$, on which the measure is the projection of the Haar measure $\chi_{G}$. Therefore, we shall believe that $\Omega=G / H$. Denote by $q$ the quotient map from $X \times G$ into $G / H$. From the definition of the associated action $W_{(\Gamma, \infty)}(G)=W(G)$ it follows that

$$
q(V(g)(x, h))=W(g) q(x, h), \quad g \in G .
$$

Besides,

$$
q(r(\alpha)(x, h))=q(x, h), \quad r(\alpha) \in \Gamma(\alpha)
$$

for a.a. $(x, h) \in X \times G$. Thus, it follows from (4.2) that there is $g_{0} \in G$ such that for a.a. $x \in X$

$$
q\left(r x, \alpha(x, r) g_{0}\right)=q\left(x, g_{0}\right), \quad r \in \Gamma
$$


Relations (2.1), (2.2) and (4.1)-(4.3) show that for a.a. $x \in X$

$$
\begin{aligned}
q\left(r x, \alpha(x, \gamma) g_{0}\right) & =W\left(g_{0}\right)^{-1} W\left(\alpha(x, r)^{-1}\right) q(r x, e), \\
q\left(x, g_{0}\right) & =W\left(g_{0}^{-1}\right) q(x, e),
\end{aligned}
$$

where $e$ is the identity in $G$. Therefore,

$$
W(\alpha(x, \gamma)) q(x, e)=q(\gamma x, e), \quad r \in \Gamma .
$$

Let $\omega_{0} \in G / H$ be the point in the quotient space into which the group $H$ is projected. Then,

$$
H=\left\{g \in G: W(g) \omega_{0}=\omega_{0}\right\} .
$$

By the theorem on the measurable choice, there exists a measurable map $\theta: G / H \rightarrow G$ such that $W(\theta(\omega)) \omega_{0}=\omega$ since $W(g)(g \in G)$ is the shift into $G / H$. For $x \in X$ we put $f(x)=\theta(q(x, e))$ and define the cocycle $\beta$, which is $\Gamma$ cohomologous to $\alpha$, by the formula: $\beta(x, \gamma)=f(\gamma x)^{-1} \alpha(x, \gamma) f(x)$. Let us check that all values of $\beta$ lie in $H$ :

$$
\begin{aligned}
W(\beta(x, \gamma)) \omega_{0} & =W\left(\theta(q(\gamma x, e))^{-1}\right) W(\alpha(x, r)) q(x, e) \\
& =W(\theta(q(r x, e)))^{-1} q(\gamma x, e)=\omega_{0} .
\end{aligned}
$$

Here we have used equalities (4.4) and (4.5). Relation (4.6) means that $\beta(x, \gamma)$ $\in H$ for a.a. $x \in X$ and all $r \in \Gamma$.

Thus, $r(\Gamma, \alpha)=H$ (assuming now $G$ to be an abelian group). From results of Section 5 (see Theorem 5.9) it immediately follows that indeed $r(\Gamma, \alpha)=H$.

4.2. Below the group $G$ is assumed to be abelian.

Theorem 4.2. Let $\Gamma \subset \operatorname{Aut}(X, \mathcal{B}, \mu)$ be an arbitrary ergodic group and $\alpha \in Z^{1}(X \times \Gamma, G)$. The action $W_{(\Gamma, \alpha)}(G)$ associated with the pair $(\Gamma, \alpha)$ is transitive if and only if the cocycle $\alpha$ is regular.

Proof. It follows from Theorem 4.1 and Lemma 1.12.

Proposition 4.3. Let there be defined a pair $(\Gamma, \alpha)$, where $\Gamma \subset \operatorname{Aut}(X, \mathcal{B}, \mu)$, $\alpha \in Z^{1}(X \times \Gamma, G)$ and $r\left(\Gamma, \alpha_{0}\right)=H_{0} \subset G_{0}$. Then, regularity of $\alpha_{0}$ means regularity of $\alpha$. The reverse is wrong.

Proof. Since $\alpha_{0}$ is regular, all values of the cocycle $\alpha_{0}$ may be thought to lie in $H_{0}$. Use, as in Section 3 , the notation $H_{0}=\left(H_{G}, H_{R}\right)$. Show that $r(\Gamma, \alpha)=\bar{H}_{G}$, where $\bar{H}_{G}$ is the closure of the group $H_{G}$ in $G$. As all values of $\alpha$ 
are in the group $H_{G}$, then evidently $r(\Gamma, \alpha) \subset \bar{H}_{G}$. Let $U$ be an arbitrary neighborhood of the identity in $G$ and $h_{0} \in \bar{H}_{G}$. Then, there is an element $h_{1} \in H_{G}$ such that $h_{1} \in h_{0}+U$. For a certain $u_{1} \in H_{R}$ the element $\left(h_{1}, u_{1}\right) \in H_{0}$. Therefore, for any $A \subset X$ and $U_{0}=U \times(-\varepsilon, \varepsilon)$ there is a subset $B \subset A$ of positive measure and an automorphism $r \in[\Gamma]$ such that $\gamma B \subset A$ and $\alpha_{0}(x, \gamma) \in\left(h_{1}, u_{1}\right)+$ $U_{0}, x \in B$. Thus, $\alpha(x, \gamma) \in h_{1}+U \subset h_{0}+2 U$ for a.a. $x \in B$, i.e. $h_{0} \in r(\Gamma, \alpha)$. Therefore, we obtain that the cocycle $\alpha$ is regular.

An example showing that regular $\alpha$ does not necessarily mean regularity of $\alpha_{0}$ will be provided in Section 7.

Corollary $4_{0} 4_{0}$ Let the pairs $\left(\Gamma_{1}, \alpha_{0}^{1}\right)$ and $\left(\Gamma_{2}, \alpha_{0}^{2}\right)$ be such that the cocycles $\alpha_{0}^{i}, i=1,2$ are regular and the associated actions $W_{\left(\Gamma_{1}, \alpha_{0}^{1}\right)}\left(G_{0}\right)$ and $W_{\left(\Gamma_{2}, \alpha_{0}^{2}\right)}\left(G_{0}\right)$ be isomorphic. Then, so are the actions $W_{\left(\Gamma_{1}, \alpha^{1}\right)}(G)$ and $W_{\left(\Gamma_{2}, \alpha^{2}\right)}(G)$.

The proof follows from Theorem 4.2 and Proposition 4.3.

The statement reverse to Corollary 4.4 is wrong.

Using the results of Section 3 (see Theorem 3.9), one can prove the uniqueness theorem for regular cocycles defined on a.f. groups of automorphisms.

Theorem 4.5. Let there be the pairs $\left(\Gamma_{1}, \alpha_{0}^{1}\right)$ and $\left(\Gamma_{2}, \alpha_{0}^{2}\right)$ such that the cocycles $\alpha_{0}^{i}, i=1,2$ are regular and let $r\left(\Gamma_{1}, \alpha_{0}^{1}\right)=r\left(\Gamma_{2}, \alpha_{0}^{2}\right)=H_{0}$ (i.e. the associated actions $W_{\left(\Gamma_{1}, \alpha_{0}^{1}\right)}\left(G_{0}\right)$ and $W_{\left(\Gamma_{2}, \alpha_{0}^{2}\right)}\left(G_{0}\right)$ be isomorphic). Then, the pairs $\left(\Gamma_{1}, \alpha_{0}^{1}\right)$ and $\left(\Gamma_{2}, \alpha_{0}^{2}\right)$ are weakly equivalent.

Proof. The statement of the theorem follows from Theorem 3.9, because $\alpha_{0, i}^{i}, 1,2$ may be thought to take values in $H_{0}$.

Remark 4.6. In [4], for an arbitrary 1.c.s. group $G$ an analogy of the set $r(\Gamma, \alpha)$ was introduced, viz. the set $\sigma(\Gamma, \alpha)$, where the cocycle $\alpha \in Z^{1}(X \times \Gamma, G)$ and $\Gamma$ is an arbitrary countable group of automorphisms. The set $\sigma(\Gamma, \alpha)$ is a closed normal subgroup of $G$ and has the same properties as $r(\Gamma, \alpha)$ (see Section 1). Theorems $4.1,4.2$ and 4.5 are true also in the assumption that $H$ is an amenable normal subgroup of $G$ (or $H_{0}$ of $G_{0}$ ). In this case, $r(\Gamma, \alpha)$ should be replaced by $\sigma(\Gamma, \alpha)$. Note also that in all the theorems of this section, the group $\Gamma$ can be of any type.

\section{§5. Free Associated Actions. Type II}

5.1. Recall our standard notation: $\Gamma$ is an ergodic a.f. countable group of automorphisms of $(X, \mathscr{B}, \mu) ; G$ a l.c.s. abclian group, and a cocycle $\alpha \in$ 
$Z^{1}(X \times \Gamma, G)$. It will be assumed that the cocycle $\alpha$ is recurrent and nonregular (see Definitions 1.11 and 1.13), because the cases of the transient and regular cocycles were considered in Sections 2 and 4. It will also be assumed that $\Gamma$ is a type II group of automorphisms. Without loss of generality it may be thought that $\Gamma$ is of type $\mathrm{II}_{1}$ and $\Gamma \circ \mu=\mu, \mu(X)=1$.

Let $r(\Gamma, \alpha)=H \subset G$. The case, where the group $H=\{0\}$, will be considered simultaneously with the general case. Section 3 treats the situation of $H=G$ and therefore $H$ will be assumed to be a closed proper subgroup of $G$. As earlier in Section 1, we shall define the cocycle $\hat{\alpha}(x, r)=\alpha(x, r)+H, r \in[\Gamma]$, which takes values in the group $\hat{G}=G / H$. By Lemma 1.14, $\hat{\alpha}$ is recurrent, as $\alpha$. By the Definition $1.11 \bar{r}(\Gamma, \hat{\alpha})=\{\hat{0}, \infty\}$, where $\hat{0}$ is the unit in $\hat{G}$.

Lemma 5.1. The pair $(\Gamma, \alpha)$ is weakly equivalent to $\left(\Gamma, \alpha_{1}\right)$ for which the cocycle $\hat{\alpha}_{1} \in Z^{1}(X \times \Gamma, \hat{G})$ is lacunary.

Proof. It follows from Lemma 1.17 that the cocycle $\hat{\alpha}$ is $\Gamma$-cohomologous to a lacunary cocycle $\hat{\alpha}_{1} \in Z^{1}(X \times \Gamma, \hat{G}): \hat{\alpha}_{1}(x, r)=\hat{f}(r x)+\hat{\alpha}(x, r)-\hat{f}(x)$, where $\hat{f}: X \rightarrow \hat{G}$ is a measurable map. According to the theorem on measurable choice, there exists a measurable map $\psi: X \rightarrow G$ such that $\hat{f}=\psi+H$. Put $\alpha_{1}(x, r)=\psi(r x)+\alpha(x, r)-\psi(x)$.

On the basis of this lemma, we shall always believe the cocycle $\hat{\alpha}$ to be lacunary. In other words, there exists a neighborhood $V_{0}$ of the identity in $\hat{G}$, for which $\hat{\alpha}(x, r) \notin V_{0}-\{\hat{0}\}, x \in X, r \in[\Gamma]$.

Consider the orbital cocycle $\hat{u}_{\hat{\alpha}}=\hat{u}$ corresponding to $\hat{\alpha}$. Let $\mathscr{R}(\Gamma)$ be the measurable equivalence relation on $X$ defined by partition of $X$ into orbits of $\Gamma$. Put $\mathcal{Q}=\left\{\left(x_{1}, x_{2}\right) \in \mathscr{R}(\Gamma): \mathfrak{u}\left(x_{1}, x_{2}\right)=0\right\}$. Obviously, $\mathscr{Q}$ is also a measurable equivalence relation and $\mathscr{Q} \subset \mathcal{R}(\Gamma)$. It follows from the results of [4] that in $[\Gamma]$ there exists an freely acting automorphism $S_{0}$ such that $\mathscr{R}\left(S_{0}\right)=\mathscr{Q}$. Denote the $\sigma$-algebra of measurable $S_{0}$-invariant subsets in $X$ by $\mathscr{B}_{0}$. Then, $\mathscr{B}_{0}$ corresponds to the partition $\xi$ of $X$ into ergodic components of $S_{0}$. Put $X_{0}=$ $X / \xi, \mu_{0}=\mu_{\xi}$ and let $\pi: X \rightarrow X_{0}$ be the natural projection onto $X_{0}$.

Lemma 5.2 [15]. Let $\mu=\int_{X_{0}} \nu_{x_{0}} d \mu_{0}\left(x_{0}\right)$ be the expansion of the measure $\mu$ into the canonical system of measures with respect to the partition $\xi$. Then, the recurrence of $\hat{\alpha}$ means that the measure $\mu_{0}$ and the measures $\left\{\nu_{x_{0}}\right\}_{x_{0} \in x_{0}}$ are probability and nonatomic for $\mu_{0}$-a.a. $x_{0} \in X_{0}$. Besides, the automorphism $S_{0}$ is conservative (i.e. not of type I), and $\nu_{x_{0}}$ is $S_{0}$-ergodic and $S_{0}$-invariant for $\mu_{0}-a, a . x_{0} \in X_{0}$. 
We consider the Lebesgue space $\left(\pi^{-1}\left(x_{0}\right), \nu_{x_{0}}\right)$ for every fixed $x_{0} \in X_{0}$. It follows from Lemma 5.2 that for $\mu_{0}$-a.a. $x_{0} \in X_{0}$ the space $\left(\pi^{-1}\left(x_{0}\right), \nu_{x_{0}}\right)$ is isomorphic to certain standard Lebesgue space $(Y, \nu), \nu(Y)=1$. Let $S\left(x_{0}\right)$ be the restriction of the automorphism $S_{0}$ onto $\pi^{-1}\left(x_{0}\right)$. According to Lemma 5.2, for $\mu_{0}$-a.a. $x_{0} \in X_{0}$, the automorphism $S\left(x_{0}\right)$ is ergodic, of type $\mathrm{II}_{1}$. From Theorem 2.5 of [10] it follows existence of a m:asurable field of isomorphisms $x_{0} \rightarrow P\left(x_{0}\right)$ $\left(x_{0} \in X_{0}\right)$ such that $P\left(x_{0}\right): Y \rightarrow \pi^{-1}\left(x_{0}\right), P\left(x_{0}\right) \circ \nu=\nu_{x_{0}}$ and $P\left(x_{0}\right)^{-1}\left[S\left(x_{0}\right)\right] P\left(x_{0}\right)=[S]$ for $\mu_{0}$-a.a. $x_{0} \in X_{0}$, where $S$ is an ergodic type $\mathrm{II}_{1}$ automorphism of $(Y, \nu)$. E.g., the space $\left(Y_{0}, p\right)$ and the automorphism group $\Delta$ of Subsection 3.1 may be taken as $(Y, \nu)$ and $S$ assuming $P$ to be $\Delta$-invariant.

Each point $x \in X$ can be represented as $x=(\pi(x), y)$, where $y \in \pi^{-1}(\pi(x))$. Then, the transformation $P: x \rightarrow\left(\pi(x), P(\pi(x))^{-1} y\right)$ maps $X$ into $X_{0} \times Y$ and the measure $\mu$ into $\mu_{0} \times \nu$. We have the automorphism group $P \Gamma P^{-1}$ and the cocycle $P \circ \alpha$, both defined on the space $\left(X_{0} \times Y, \mu_{0} \times \nu\right)$. Thus, the pair $(\Gamma, \alpha)$ transforms into $\left(P \Gamma P^{-1}, P \circ \alpha\right)$. For simplicity, we shall believe that on $\left(X_{0} \times Y, \mu_{0} \times \nu\right)$ the automorphism group $\Gamma$ acts with the cocycle $\alpha \in Z^{1}\left(X_{0} \times Y \times\right.$ $\Gamma, G)$, and these have the following properties: $S_{0}:\left(x_{0}, y\right) \rightarrow\left(x_{0}, S y\right)$ belongs to $[\Gamma]$, and $\hat{\alpha}\left(x_{0}, y, S_{0}\right)=\hat{0}$.

Lemma 5.3 [15]. On the Lebesgue space $\left(X_{0}, \mathcal{B}_{0}, \mu_{0}\right)$, there exists an a.f. countable ergodic group $\Gamma_{0}$ of automorphisms such that

$$
\pi\left(\Gamma_{X}\right)=\Gamma_{\mathrm{n}} \pi(x), x \in X=X_{0} \times Y,
$$

and there exists an orbital transient cocycle $u_{0}: \mathscr{R}\left(\Gamma_{0}\right) \rightarrow \hat{G}$ such that for any $r \in[\Gamma]$

$$
\hat{\alpha}(x, \gamma)=u_{0}(\pi(\gamma x), \pi(x)), \quad x \in X .
$$

Relations (5.1) and (5.2) imply the following. The orbits of $\Gamma_{0}$ is formed by the projection $\pi$ of the orbits of $\Gamma$ onto $X_{0}$. The cocycle $u_{0}$ has the same set of values as the cocycle $\hat{\alpha}$. Therefore, there exists a neighborhood $V_{0}$ of the identity in $\hat{G}$ for which $u_{0}\left(r_{0} x_{0}, x_{0}\right) \notin V_{0}, r_{0} \in\left[\Gamma_{0}\right]\left(r_{0} \neq 1\right), x_{0} \in X_{0}$. From Lemma 5.3 it follows that there exists an ergodic automorphism $Q \in \operatorname{Aut}\left(X_{0}, \mathscr{B}_{0}, \mu_{0}\right)$ for which $\left[\Gamma_{0}\right]=[Q]$. Put $\varphi\left(x_{0}\right)=u_{0}\left(Q x_{0}, x_{0}\right), x_{0} \in X_{0}$.

Lemma 5.4. In the full group $[\Gamma]$ there exists an automorphism $R$, such that

$$
\hat{\alpha}(x, R)=\varphi(\pi(x)), \quad x \in X .
$$

Proof. Since all elements of $\Gamma_{0}$ result from projection of $\Gamma$ onto $X_{0}$, then 
for the automorphism $Q \in\left[\Gamma_{0}\right]$ there exists an automorphism $R \in[\Gamma]$ such that

$$
R:\left\{x_{0}\right\} \times Y \rightarrow\left\{Q x_{0}\right\} \times Y, x_{0} \in X_{0} .
$$

By using equality (5.2), we obtain it that $\hat{\alpha}(x, R)=u_{0}(\pi(R x), \pi(x))=u_{0}(Q \pi(x)$, $\pi(x))=\varphi(\pi(x))$.

Consider the automorphism

$$
R\left(Q^{-1} \times \mathbf{1}\right):\left(x_{0}, y\right) \rightarrow\left(x_{0}, y^{\prime}\right),
$$

which for a.a. $x_{0} \in X_{0}$ defines a measurable one-to-one map $U\left(x_{0}\right): y-y^{\prime}$. Relation (5.5) shows that $x_{0} \rightarrow U\left(x_{0}\right)\left(x_{0} \in X_{0}\right)$ is the measurable field of automorphisms of $(Y, \nu)$.

Lemma 5.5. The following statements are true: $R \in N\left[S_{0}\right]$ and $U\left(x_{0}\right) \in N[S]$ for $\mu_{0}$-a.a. $x_{0} \in X_{0}$.

Proof. By using (5.3), (5.4) and (5.5), we obtain that $\hat{\alpha}\left(x, R S_{0} R^{-1}\right)=\hat{0}$. Therefore, $R S_{0} R^{-1} \in\left[S_{0}\right]$. Then, $R\left(Q^{-1} \times 1\right) \in N\left[S_{0}\right]$, which, together with (5.5), leads to $U\left(x_{0}\right) \in N[S], x_{0} \in X_{0}$.

Introduce the notation: $Q_{0}\left(x_{0}, y\right)=\left(Q x_{0}, U\left(x_{0}\right) y\right)$. Unite now the above proved results in the following theorem on the structure of the cocycles $\hat{\alpha}$.

Theorem 5.6. Let a pair $(\Gamma, \alpha)$ be as above. The full group $[\Gamma]$ is generated by the action of two automorphisms $Q_{0}$ and $S_{0}$ on $\left(X_{0} \times Y, \mu_{0} \times \nu\right)$ as follows $Q_{0}\left(x_{0}, y\right)=\left(Q x_{0}, U\left(x_{0}\right) y\right), S_{0}\left(x_{0}, y\right)=\left(x_{0}, S y\right)$ with $Q_{0} \in N\left[S_{0}\right] . \quad$ The cocycle $\hat{\alpha} \in$ $Z^{1}(X \times \Gamma, G)$ has the properties: $\hat{\alpha}\left(x_{0}, y, Q_{0}\right)=\varphi(x), \hat{\alpha}\left(x_{0}, y, S_{0}\right)=\hat{0}$, where $\varphi\left(x_{0}\right) \notin$ $V_{0}\left(V_{0}\right.$ is a neighborhood of the identity in $\left.\hat{G}\right)$. The set of values of $\hat{\alpha}$ coincides with that of the orbital cocycle $u_{0}$.

Proof. We have to prove only the equality

$$
\{r x: r \in \Gamma\}=\left\{Q_{0}^{m} S_{0}^{k}\left(x_{0}, y\right): m, k \in Z\right\}, x=\left(x_{0}, y\right),
$$

since all the other statements of the theorem follow from Lemmas 5.2-5.5. Assume (5.6) to be wrong; i.e. the set

$$
A=\bigcup_{\gamma \in \Gamma}\left\{x \in X: r x \notin\left\{Q_{0}^{m} S_{0}^{k} x: m, k \in Z\right\}\right\}
$$

has a positive measure. As has been said, the cocycle $\alpha$ (and thus $\hat{\alpha}$ ) may be thought to take on only a countable number of values. The condition of the theorem means that for any fixed $r \in \Gamma$ there exists a measurable function 
$m=m(\pi(x))$ for which

$$
\hat{\alpha}(x, r)=u_{0}\left(Q^{m(\pi(x))} \pi(x), \pi(x)=\hat{\alpha}\left(x, Q_{0}^{m(\pi(x))}\right) .\right.
$$

Then, there is an automorphism $\gamma_{1}=\gamma_{1}(x) \in[\Gamma]$ such that $r x=\gamma_{1} Q_{0}^{m} x$ and for $x \in A$

$$
\hat{\alpha}(x, \gamma)=\hat{\alpha}\left(Q_{0}^{m} x, \gamma_{1}\right)+\hat{\alpha}\left(x, Q_{0}^{m}\right) .
$$

Comparing (5.7) and (5.8), we conclude that $\hat{\alpha}\left(Q_{0}^{m} x, r_{1}\right)=\hat{0}$, i.e. $\gamma_{1} \in\left[S_{0}\right]$; this is contradiction to the above assumption and thus proves (5.6).

5.2. Here it will be assumed that the cocycle $\alpha \in Z^{1}(X \times \Gamma, G)$ is lacunary (it is also recurrent and nonregular). This means that $\bar{r}(\Gamma, \alpha)=\{0, \infty\}$. Therefore, all the results formulated and proved for $\hat{\alpha}$ in Subsection 5.1 are also true for $\alpha$ because $\hat{\alpha}=\alpha$ for the group $H=\{0\}$.

Let us first consider the case where the action $W_{(\Gamma, \alpha)}(G)$ associated with the pair $(\Gamma, \alpha)$ preserves measure.

Lemma 5.7. Let $\Gamma$ be an ergodic a.f. type $\mathbb{I I}_{1}$ group of automorphisms of $(X, \mathscr{B}, \mu)$, let $\alpha \in Z^{1}(X \times \Gamma, G)$ be a lacunary cocycle, and the action $W_{(\Gamma, \alpha)}(G)$ preserves measure. Then, in the condition of Theorem 5.6 the automorphism $Q_{0}$ may be chosen to be $Q \times 1$.

Proof. We shall calculate the associated action $W_{(\Gamma, \alpha)}(G)$. From the ergodicity of $S$ on $(Y, \nu)$ and the triviality of $\alpha$ on $S_{0}$, it follows that the action $W_{(\Gamma, \infty)}(G)$ is defined on the quotient space of $\left(X_{0} \times G, \mu_{0} \times x_{G}\right)$ by the partition into orbits of the automorphism $Q\left(u_{0}\right):\left(x_{0}, g\right) \rightarrow\left(Q x_{0}, g+u_{0}\left(Q x_{0}, x_{0}\right)\right)$. This partition is measurable, because the cocycle $u_{0}$ on $Q_{0}$ is transient and $\alpha\left(x, Q_{0}\right)=$ $u_{0}\left(Q x_{0}, x_{0}\right)=\varphi\left(x_{0}\right) \notin V_{0}$, where $V_{0}$ is a certain neighborhood of the identity in $G$. Hence, $W_{(\Gamma, \alpha)}(G)=W_{\left(Q, u_{0}\right)}(G)$. The action $W_{\left(Q, u_{0}\right)}(G)$ preserves measure if and only if $Q$ preserves measure $\mu_{0}$. Now, since the probability measure $\nu$ on $Y$ is $S$-invariant and $S$-ergodic, then the condition $U\left(x_{0}\right) \in N[S]$ means that $U\left(x_{0}\right) \circ \nu$ $=\nu$ for $\mu_{0}$-a.a. $x_{0} \in X_{0}$. Applying the cohomology theorem [10] (see also [1]), we obtain existence of a measurable field of automorphisms $x_{0} \rightarrow P\left(x_{0}\right) \in N[S]$ such that $P\left(Q x_{0}\right)^{-1} U\left(x_{0}\right) P\left(x_{0}\right) \in[S], x_{0} \in X_{0}$. This means that the transformation $P:\left(x_{0}, y\right) \rightarrow\left(x_{0}, P\left(x_{0}\right) y\right)$ maps $[T]$ into the group generated by $Q \times \mathbb{1}=Q_{0}$ and $\mathbb{1} \times S=S_{0}$. This does not change the cocycle $\alpha$.

The proofs of Lemma 5.7 and Theorem 2.9 lead to

Proposition 5.8. Let the pairs $\left(\Gamma_{\dot{\xi}}, \alpha_{\dot{z}}\right), i=1,2$ satisfy the following condi- 
tions: $\Gamma_{i}$ is an a.f. countable ergodic type II group of automorphisms of $\left(X_{i}, \mathscr{B}_{i}, \mu_{i}\right), \alpha_{i} \in Z^{1}\left(X_{i} \times \Gamma_{i}, G\right)$ is a lacunary cocycle, and the action $W_{i}(G)=$ $W_{\left(\Gamma_{i}, \alpha_{i}\right)}(G)$ preserves measure. The actions $W_{1}(G)$ and $W_{2}(G)$ are isomorphic if and only if the pairs $\left(Q^{1}, u_{0}^{1}\right)$ and $\left(Q^{2}, u_{0}^{2}\right)$ are stably weakly equivalent, where $\left(Q^{i}, u_{0}^{i}\right), i=1,2$ are defined by $\left(\Gamma_{i}, \alpha_{i}\right)$, as in Theorem 5.6.

Theorem 5.9. (theorem of uniqueness). Let the pairs $\left(\Gamma_{i}, \alpha_{i}\right), i=1,2$ be the same as in Proposition 5.8 and the associated action $W_{i}(G)=W_{\left(\Gamma_{i}, \alpha_{i}\right)}(G)$ preserves measure. The pairs $\left(\Gamma_{1}, \alpha_{1}\right)$ and $\left(\Gamma_{2}, \alpha_{2}\right)$ are stably weakly equivalent if and only if $W_{1}(G)$ and $W_{2}(G)$ are isomorphic.

Proof. Obviously, we may take $X_{1}=X_{2}=X$. Then, it follows from Theorem 5.6 that $Q^{1}=Q^{2}=Q, X_{0}^{1}=X_{0}^{2}=X_{0}, \mu_{0}^{1}=\mu_{0}^{2}=\mu_{0}, Y_{1}=Y_{2}=Y, \nu_{1}=\nu_{2}=\nu, S^{1}=S^{2}$ $=S$. The pairs $\left(\Gamma_{1}, \alpha_{1}\right)$ and $\left(\Gamma_{2}, \alpha_{2}\right)$ differ only in the values of $\alpha_{1}$ and $\alpha_{2}$ on $Q_{0}=Q \times 1$. The pairs $\left(\Gamma_{1}, \alpha_{1}\right)$ and $\left(\Gamma_{2}, \alpha_{2}\right)$ are stably equivalent if and only if so are $\left(Q, u_{0}^{1}\right)$ and $\left(Q, u_{0}^{2}\right)$. Indeed, it follows from the structure of $\alpha_{1}$ and $\alpha_{2}$ that if $\psi_{0}: X_{0} \times \boldsymbol{Z} \rightarrow X_{0} \times \boldsymbol{Z}$ is a map responsible for weak equivalence of the cocycles $\tilde{u}_{0}^{1}$ and $\tilde{u}_{0}^{2}$, then the map $\psi:\left(x_{0}, n, y\right) \rightarrow\left(\psi_{0}(x, n), y\right)$ will define weak equivalence of the pairs $\left(\tilde{\Gamma}_{1}, \tilde{\alpha}_{1}\right)$ and $\left(\tilde{\Gamma}_{2}, \tilde{\alpha}_{2}\right)$ (recall that $\tilde{u}_{0}^{i}$ and $\tilde{\alpha}_{i}$ are the countable expansions of $u_{0}^{i}$ and $\left.\alpha_{i}, i=1,2\right)$. Therefore, by Proposition 5.8, the isomorphism of $W_{1}(G)$ and $W_{2}(G)$ means stable weak equivalence of $\left(\Gamma_{1}, \alpha_{1}\right)$ and $\left(\Gamma_{2}, \alpha_{2}\right)$. The reverse statement was proved in Proposition 2.3.

Let us show now that any free measure-preserving action $W(G)$ of $G$ may be regarded as associated with a pair $(\Gamma, \alpha)$, where $\Gamma$ is a type II group and $\alpha$ a lacunary cocycle. Namely, we shall prove

Theorem 5.10 (theorem of existence). Let $W(G)$ be an ergodic free action of $G$ on a Lebesgue space $(\Omega, p)$ preserving the probability measure $p$. Then, there exists an ergodic countable a.f. group $\Gamma \subset \operatorname{Aut}(X, \mathscr{B}, \mu), \mu(X)=1$, preserving the measure $\mu$, and there exists a lacunary cocycle $\alpha \in Z^{1}(X \times \Gamma, G)$, both such that the action $W(G)$ is isomorphic to the action $W_{(\Gamma, \infty)}(G)$ associated with the pair $(\Gamma, \alpha)$.

Proof. Choose a complete lacunary Borel section $X_{0} \subset \Omega$ of the action $W(G)$. The measure $\mu_{0}$ on $X_{0}$ will be defined as the image of the measure $p$. There exists on $X_{0}$ a countable ergodic equivalence relation $\mathscr{R}$, and let $Q$ be such an automorphism of $X_{0}$ that $\mathscr{R}(Q)=\mathscr{R}$ [4]. Clearly, $Q$ preserves the measure $\mu_{0}$, and $\mu_{0}\left(X_{0}\right)=1$. Define the return cocycle $u_{0} \in Z^{1}\left(X_{0} \times[Q], G\right)$ for the action $Q$ on $X_{0}$, assuming $u_{0}\left(Q x_{0}, x_{0}\right)=g$, where $g \in G$ satisfies the equality 
$W(g) x_{0}=Q x_{0}$. Since the action $W(G)$ is free, then $g=g\left(x_{0}\right)$ is defined unambiguously.

Let $(Y, \nu), \nu(Y)=1$ be a Lebesgue space, and $S \in \operatorname{Aut}(Y, \nu)$ be an ergodic automorphism preserving $\nu$. Define on $(X, \mu)=\left(X_{0} \times Y, \mu_{0} \times \nu\right)$ an group $\Gamma$ of automorphisms of type $\Pi_{1}$ generated by $Q_{0}$ and $S_{0}$ :

$$
Q_{0}\left(x_{0}, y\right)=\left(Q x_{0}, y\right), \quad S_{0}\left(x_{0}, y\right)=\left(x_{0}, S y\right) .
$$

The group $\Gamma$ is ergodic and a.f. Put

$$
\alpha\left(x_{0}, y, Q_{0}\right)=u_{0}\left(Q x_{0}, x_{0}\right), \alpha\left(x_{0}, y, S_{0}\right)=0 \text { 。 }
$$

Since the section $X_{0}$ is lacunary, then the cocycle $u_{0}$ is transient and the cocycle $\alpha$ is lacunary. It is now transparent that the associated action $W_{(\Gamma, \alpha)}(G)$ is isomorphic to $W(G)$ (see e.g. [3] and also Section 2).

There exists an example of a type II group $\Gamma$ and a lacunary cocycle $\alpha \in Z^{1} /(X \times \Gamma, G)$ such that the associated action $W_{(\Gamma, \alpha)}(G)$ is non-singular (i.e. of type III). Moreover, such a pair $(\Gamma, \alpha)$ may be constructed by any nonsingular action $W(G)$ of $G$.

Theorem 5.11. Let $W(G)$ be an ergodic free action of $G$ on an Lebesgue space $(\Omega, p), p(\Omega)=1$ with a non-singular measure $p$. Then, there exists a piar $(\Gamma, \alpha)$, where $\Gamma$ is an ergodic a.f. group of automorphisms of $(X, \mathscr{B}, \mu)$ preserving a $\sigma$-finite measure $\mu$ and $\alpha \in Z^{1}(X \times \Gamma, G)$ is a lacunary cocycle, and this pair is such that $W_{(\Gamma, \infty)}(G)$ and $W(G)$ are isomorphic.

Proof. As in the proof of Theorem 5.10, let us define the following objects: $\left(X_{0}, \mu_{0}\right), Q, \mathscr{R}$ and $u_{0}$. The automorphism $Q$ has, generally speaking, the non-trivial Radon-Nikodym cocycle $\rho\left(x_{0}, Q\right)=\log \frac{d Q_{0}^{-1} \circ \mu_{0}}{d \mu_{0}}\left(x_{0}\right)$. Consider a Lebesgue space $(Y, \nu)$ with $\sigma$-finite measure $\nu$ and an ergodic automorphism $S$ of $(Y, \nu)$ preserving $\nu$. Let $x_{0} \rightarrow U\left(x_{0}\right)\left(x_{0} \in X_{0}\right)$ be a measurable field of automorphisms of $(Y, \nu)$ such that $U\left(x_{0}\right) \in N[S]$ and

$$
\Phi\left(U\left(x_{0}\right)\right) \equiv \log \frac{d U\left(x_{0}\right)^{-1} \circ \nu}{d \nu}=-\rho\left(x_{0}, Q\right) .
$$

Put $(X, \mu)=\left(X_{0} \times Y, \mu_{0} \times \nu\right)$ and $Q_{0}\left(x_{0}, y\right)=\left(Q x_{0}, U\left(x_{0}\right) y\right), S_{0}\left(x_{0}, y\right)=\left(x_{0}, S y\right)$. Then, $Q_{0} \in N\left[S_{0}\right]$. Denote by $\Gamma$ the a.f. type $\mathbb{I}_{\infty}$ group of automorphisms generated by $Q_{0}$ and $S_{0}$. Define the cocycle $\alpha$ for $\Gamma$, according to formulae (5.10). The pair $(\Gamma, \alpha)$ will satisfy the conditions of the theorem. 
Let us prove now the uniqueness theorem for the pairs $(\Gamma, \alpha)$, whose associated actions are non-singular.

Theorem 5.12. Let $\Gamma_{i}$ be an ergodic a.f. type II group of automorphisms of $\left(X_{i}, \mathcal{B}_{i}, \mu_{i}\right)$, and $\alpha_{i} \in Z^{1}\left(X_{i} \times \Gamma_{i}, G\right)$ be a lacunary cocycle, $i=1,2$. Assume $W_{\left(\Gamma_{i}, \alpha_{i}\right)}(G)$ associated with $\left(\Gamma_{i}, \alpha_{i}\right)$ to be non-singular, $i=1,2$. The pairs $\left(\Gamma_{1}, \alpha_{1}\right)$ and $\left(\Gamma_{2}, \alpha_{2}\right)$ are stably weakly equivalent if and only if $W_{\left(\Gamma_{1}, \alpha_{1}\right)}(G)$ and $W_{\left(\Gamma_{2}, \alpha_{2}\right)}(G)$ are isomorphic.

Proof. Since we are interested in the stable weak equivalence relation, then we may consider the pair $\left(\tilde{\Gamma}_{i}, \widetilde{\alpha}_{i}\right)$ instead of $\left(\Gamma_{i}, \alpha_{i}\right), i=1,2$, i.e. believe that $\Gamma_{i}$ is of type $\mathrm{II}_{\infty}$ and $\mu_{i}$ is $\Gamma_{i}$-invariant and infinite. Theorem 5.6 naturally remains valid in this case as well, and the measure $\nu$ on the space $Y$ is also infinite (see Subsection 5.1). The automorphism $Q_{0}^{i}\left(x_{0}, y\right)=\left(Q^{i} x_{0}, U_{i}\left(x_{0}\right) y\right)$ preserves the measure $\mu_{i}=\mu_{0}^{i} \times \nu$, therefore, $\Phi\left(U_{i}\left(x_{0}\right)\right)=-\rho\left(x_{0}, Q^{i}\right)$ (here $\left(x_{0}, y\right)$ $\in X)$. Based on the isomorphism of $W_{\left(\Gamma_{1}, \alpha_{1}\right)}(G)$ and $W_{\left(\Gamma_{2}, \alpha_{2}\right)}(G)$, it may be assumed, as in Theorem 5.9, that $\Gamma_{1}=\Gamma_{2}=\Gamma, Q^{1}=Q^{2}=Q, \alpha_{1}=\alpha_{2}=\alpha,\left(X_{1}, \mu_{1}\right)=$ $\left(X_{2}, \mu_{2}\right)=(X, \mu)$. Thus, $\Phi\left(U_{1}\left(x_{0}\right)\right)=\Phi\left(U_{2}\left(x_{0}\right)\right)$. By the cohomology theorem [10] there exists a measurable field of automorphisms $x_{0} \rightarrow P\left(x_{0}\right)\left(x_{0} \in X_{0}\right)$ such that $P\left(x_{0}\right) \in N[S]$ and for a.a. $x_{0} \in X_{0}$

$$
P\left(Q x_{0}\right)^{-1} U_{1}\left(x_{0}\right) P\left(x_{0}\right)=U_{2}\left(x_{0}\right) s\left(x_{0}\right),
$$

where $x_{0} \rightarrow s\left(x_{0}\right) \in[S]$. Equality (5.12) shows that the transformation $P:\left(x_{0}, y\right)$ $\rightarrow\left(x_{0}, P\left(x_{0}\right) y\right)$ belongs to $N[\Gamma]$ and $P \circ \alpha=\alpha$, because the cocycle $\alpha$ is completely defined by the action of $Q$ on $X_{0}$. Thus, $P$ maps the generators $\left(Q_{0}^{1}, S_{0}\right)$ of $[\Gamma]$ into the generators $\left(Q_{0}^{2}, S\right)$ without $\alpha$ being replaced. This proof is concluded, as that of Theorem 5.9, by consideration of the corresponding transient cocycles.

Corollary 5.13. Let $\alpha \in Z^{1}(X \times \Gamma, G)$ be a lacunary cocycle, where $\Gamma$ is of type II. There exists a cocycle $\alpha_{1}$, which is stably weakly equivalent to $\alpha$ and such that $\alpha_{1}$ is trivial on $S_{0}$ and $\alpha_{1}$ is a transient cocycle on $Q_{0}$, where $Q_{0}$ and $S_{0}$ are the generators of $[\Gamma]$.

\section{§6. Non-free Associated Actions. Type II}

6.1. Let $\Gamma$ be an ergodic a.f. group of automorphisms, $G_{\mathbf{0}}=G \times \boldsymbol{R}$, and a cocycle $\alpha_{0} \in Z^{1}\left(X \times \Gamma, G_{0}\right)$. Assume $\alpha_{0}$ to be nonregular and $r\left(\Gamma, \alpha_{0}\right)=H_{0}$, and $\Gamma$ to be of type III. If $\Gamma$ is a type II group, then the proofs below, will be 
simplified.

Recall that, according to Theorem 5.6, $\Gamma$ is generated by $Q_{n}$ and $S_{0}$ which act on the Lebesgue space $\left(X_{0} \times Y, \mu_{0} \times \nu\right)$ as follows

$$
Q_{0}\left(x_{0}, y\right)=\left(Q x_{0}, U\left(x_{0}\right) y\right), \quad S_{0}\left(x_{0}, y\right)=\left(x_{0}, S y\right) .
$$

The cocycle $\hat{\alpha} \in Z^{1}\left(X_{0} \times Y \times \Gamma, \hat{G}_{0}\right), \hat{G}_{0}=G_{0} / H_{0}$ takes on $Q_{0}$ and $S_{0}$ the following values

$$
\hat{\alpha}_{0}\left(x_{0}, y, Q_{0}\right)=\varphi\left(x_{0}\right), \quad \hat{\alpha}_{0}\left(x_{0}, y, S_{0}\right)=\hat{0},
$$

where $\varphi\left(x_{0}\right) \notin V_{0}$ and $V_{0}$ is a neighborhood of identity in $\hat{G}_{0}$.

It follows from (6.2) that the cocycle $\alpha_{0}$ takes the values on $S_{0}$ from the group $H_{0}$. Thus, $\alpha_{0}$ on $\left[S_{0}\right]$ defines a measurable field of cocycles $x_{0} \rightarrow \alpha_{0}\left(x_{0}\right)$ $(y, s), s \in\left[S_{0}\right], x_{0} \in X_{0}$ with values in $H_{0}$.

Lemma 6.耳. The range of the field of cocycles $x_{0} \rightarrow \alpha_{0}\left(x_{0}\right)(y, s), s \in[S]$ is the group $H_{0}$.

Proof. It follows from (6.2) that there exists such a neighborhood $V$ of the identity in $G_{0}$ that the values of $\alpha_{0}$ on $\Gamma$ do not belong to the set $\left(H_{0}+V\right)-H_{0}$. Let $h_{0}$ be an arbitrary element of $H_{0}$ and $W$ an arbitrary neighborhood of the identity in $G_{0}$. The condition $r\left(\Gamma, \alpha_{0}\right)=H_{0}$ means that for any set $A \subset X_{0} \times Y_{\text {, }}$ $\left(\mu_{0} \times \nu\right)(A)>0$ there exist a subset $B \subset A_{,}\left(\mu_{0} \times \nu\right)(B)>0$ and an automorphism $r \in[\Gamma]$, such that $r B \subset A$ and $\alpha_{0}\left(x_{0}, y, \gamma\right) \in h_{0}+W$ for a.a. $\left(x_{0}, y\right) \in B$. Since $\gamma\left(x_{0}, y\right)=S_{0}^{m} Q_{0}^{n}\left(x_{0}, y\right)$, where $m=m\left(x_{0}, y\right), n=n\left(x_{0}, y\right)$, then we obtain for $\left(x_{0}, y\right)$ $\in B$

$$
\alpha_{0}\left(Q_{0}^{n}\left(x_{0}, y\right), S_{0}^{m}\right)+\alpha_{0}\left(x_{0}, y, Q_{0}^{n}\right) \in h_{0}+W .
$$

Since $\alpha_{0}$ on $Q_{0}$ takes values in $H_{0}$, then $\alpha_{0}\left(x_{0}, y, Q_{0}^{n}\right) \in H_{0}+W$. Let $W \subset V$; we obtain that $\alpha_{0}\left(x_{0}, y, Q_{0}^{n}\right) \in H_{0}$. It follows from (6.2) that in this case $n=0$ and then $r\left(x_{0}, y\right)=S_{0}^{m}\left(x_{0}, y\right)$. Hence, the field of cocycles $x_{0} \rightarrow \alpha_{0}\left(x_{0}\right)\left(x_{0} \in X_{0}\right)$ has the property $r\left(\left\{\alpha_{0}(\cdot)\right\}\right)=H_{0}$.

The above properties of $\Gamma$ and $\alpha_{0}$ show that the ergodic automorphism $S \in \operatorname{Aut}(Y, \mathscr{F}, \nu)$ may be only either of type $\mathbb{I I}$ or of type $\mathbb{I I I}_{\lambda}(0<\lambda \leq 1)$. This depends on the group $H_{R}$, where $H_{0}=\left(H_{G}, H_{R}\right)$ (see Subsection 3.1). In Section 3 we introduced the standard cocycle $\beta_{0}$ defined on the a.f. group of automorphisms, its range coinciding with $H_{0}$. Application of Theorem 3.13 yields the following result: there exists a measurable field of automorphisms $x_{0} \rightarrow R\left(x_{0}\right) \in$ $N[S]$ such that the cocycle $R \circ \alpha_{0}$ is $S_{0}$-cohomologous to a constant field of cocy- 
les $\beta_{0}$, where $R\left(x_{0}, y\right)=\left(x_{0}, R\left(x_{0}\right) y\right)$. In other words, there exists a measurable function $f: X_{0} \times Y \rightarrow H_{0}$ such that for $s_{0} \in\left[S_{0}\right],\left(x_{0}, y\right) \in X_{0} \times Y$

$$
f\left(s_{0}\left(x_{0}, y\right)\right)+R \circ \alpha_{0}\left(x_{0}, y, s_{0}\right)-f\left(x_{0}, y\right)=\beta_{0}\left(y, s\left(x_{0}\right)\right),
$$

where $s_{0}\left(x_{0}, y\right)=\left(x_{0}, s\left(x_{0}\right) y\right)$. Under the action of $R$ the group $\Gamma$ will transform into the group $\Gamma^{\prime}=R \Gamma R^{-1}$ generated by $S_{0}$ and $Q_{0}^{\prime}$, where $Q_{0}^{\prime}\left(x_{0}, y\right)=$ $\left(Q x_{0}, U^{\prime}\left(x_{0}\right) y\right), U^{\prime}\left(x_{0}\right)=R\left(Q x_{0}\right) U\left(x_{0}\right) R\left(x_{0}\right)^{-1}$. Since $R$ preserves the measure $\nu$ (see Section 3), then for a.a. $x_{0} \in X_{0}, \Phi\left(U\left(x_{0}\right)\right)=\Phi\left(U^{\prime}\left(x_{0}\right)\right)$. The cocycle $\alpha_{0}$ will be replaced by $\alpha_{0}^{\prime}$ defined on $\Gamma^{\prime}$ :

$$
\alpha_{0}^{\prime}\left(x_{0}, y, r^{\prime}\right)=f\left(r^{\prime}\left(x_{0}, y\right)\right)+R \circ \alpha_{0}\left(x_{0}, y, r^{\prime}\right)-f\left(x_{0}, y\right),
$$

where $f$ is the same as in (6.3), i.e. $\alpha_{0}^{\prime}$ coincides with $\beta_{0}$ on $\left[S_{0}\right]$. Thus, we have proved the following

Lemma 6.2. The pair $\left(\Gamma, \alpha_{0}\right)$, where $r\left(\Gamma, \alpha_{0}\right)=H_{0}$, is weakly equivalent to the pair $\left(\Gamma^{\prime}, \alpha_{0}^{\prime}\right)$ for which $\alpha_{0}^{\prime}\left(x_{0}, y, S_{0}\right)=\beta_{0}\left(y, S_{0}\right)$.

Consider first the value of $\alpha_{0}^{\prime}$ on $Q_{0}^{\prime}$. Let $\sigma: G_{0} / H_{0} \rightarrow G_{0}$ be a measurable section of $G_{0}$ over $G_{0} / H_{0}$. This means that $\sigma(\hat{g}) \in G_{0}$ and $\pi(\sigma(\hat{g}))=\hat{g}$, where $\hat{g} \in \hat{G}_{0}$ and $\pi: G_{0} \rightarrow \hat{G}_{0}$ is a natural projection. Thus, any element $g_{0} \in G_{0}$ can be represented as

$$
g_{0}=\sigma\left(\hat{g}_{0}\right)+h_{0}\left(g_{0}\right),
$$

where $h_{0}\left(g_{0}\right) \in H_{0}$. We have, in view of (6.2) and (6.5) $\hat{\alpha}_{0}\left(x_{0}, y, Q_{0}\right)=\sigma\left(\varphi\left(x_{0}\right)\right)+$ $h_{0}\left(x_{0}, y\right)$, where $h_{0}\left(x_{0}, y\right)$ is a measurable function from $X_{0} \times Y$ into $H_{0}$. The value of $R \circ \alpha_{0}$ on $Q_{0}^{\prime}$ is easy to calculate: $R \circ \alpha_{0}\left(x_{0}, y, Q_{0}^{\prime}\right)=\sigma\left(\varphi\left(x_{0}\right)\right)+h_{0}\left(x_{0}, R\left(x_{0}\right)^{-1} y\right)$. Then, according to (6.4),

$$
\begin{aligned}
\alpha_{0}^{\prime}\left(x_{0}, y, Q_{0}^{\prime}\right)= & f\left(Q x_{0}, U^{\prime}\left(x_{0}\right) y\right)+\sigma\left(\varphi\left(x_{0}\right)\right) \\
& +h_{0}\left(x_{0}, R\left(x_{0}\right)^{-1} y\right)-f\left(x_{0}, y\right) .
\end{aligned}
$$

Lemma 6.3. Let $l\left(x_{0}, y\right)=-f\left(Q x_{0}, U^{\prime}\left(x_{0}\right) y\right)-h_{0}\left(x_{0}, R\left(x_{0}\right)^{-1} y\right)+f\left(x_{0}, y\right)$. Then, in $\left[S_{0}\right]$ there exists a measurable field of automorphisms $s_{0}=\left(x_{0} \rightarrow s\left(x_{0}\right)\right)$ such that for a.a. $\left(x_{0}, y\right) \in X_{0} \times Y$

$$
\alpha_{0}^{\prime}\left(Q_{0}^{\prime}\left(x_{0}, y\right), s_{0}\right)=l\left(x_{0}, y\right) .
$$

The proof of the lemma is transparent enough, so we shall only provide a sketch of it. As earlier, taking into consideration the results of [6], the cocycle $\alpha_{0}$ may be assumed to take values in a countable group $H_{0}^{\prime}$ which is dense in 
$H_{0}$. In this case the function $f$ can be chosen so that $\alpha_{0}^{\prime}$ should be aslo take values in $H_{0}^{\prime}$ (see (6.4) and Remark 3.12). Therefore, $f\left(r^{\prime}\left(x_{0}, y\right)\right)-f\left(x_{0}, y\right)$ belongs to $H_{0}^{\prime}$. These observations enable conclusion that the function $l$ is piecewise constant and has values in $H_{0}^{\prime}$. Since the group $\left[S_{\beta}\right]=\left\{s \in[S], \beta_{0}\left(y_{9} s\right)\right.$ $=0, y \in Y\}$ is ergodic on $(Y, \nu)$, then equality (6.7) is easy to obtain for any fixed $x_{0} \in X_{0}$. Because $l\left(x_{0}, y\right)$ is the measurable function, the corresponding field of automorphisms $s_{0}=\left(x_{0} \rightarrow s\left(x_{0}\right)\right)$ may be chosen to be measurable.

Put $\bar{Q}_{0}^{\prime}\left(x_{0}, y\right)=\left(Q x_{0}, s\left(Q x_{0}\right) U^{\prime}\left(x_{0}\right) y\right)=s_{0} Q_{0}^{\prime}\left(x_{0}, y\right)$, where $s_{0}$ is the same as in Lemma 6.3. Then, it follows from (6.6) and (6.7) that $\alpha_{0}^{\prime}\left(x_{0}, y, \bar{Q}_{0}^{\prime}\right)=$ $\sigma\left(\varphi\left(x_{0}\right)\right) \equiv \bar{\varphi}\left(x_{0}\right)$. Thereby the following result is proved.

Theorem 6.4. For a pair $\left(\Gamma, \alpha_{0}\right)$ having the properties described in the beginning of Subsection 6.1 , there exists a pair $\left(\Gamma^{\prime}, \alpha_{0}^{\prime}\right)$ weakly equivalent to $\left(\Gamma, \alpha_{0}\right)$ and such that $\Gamma^{\prime}$ is generated by $\bar{Q}_{0}^{\prime}$ and $S_{0}$, so that

$$
\alpha_{0}^{\prime}\left(x_{0}, y, \bar{Q}_{0}^{\prime}\right)=\bar{\varphi}\left(x_{0}\right), \quad \alpha_{0}^{\prime}\left(x_{0}, y, S_{0}\right)=\beta_{0}(y, S) .
$$

6.2. We shall calculate the associated action $W_{\left(\Gamma, \alpha_{0}\right)}\left(G_{0}\right)$ of $G_{0}$, where $\left(\Gamma, \alpha_{0}\right)$ is the same as in Subsection 6.1.

Proposition 6.5. For a pair $\left(\Gamma, \alpha_{0}\right), r\left(\Gamma, \alpha_{0}\right)=H_{0}$, the associated action $W_{\left(\Gamma, \alpha_{0}\right)}\left(G_{0}\right)$ has the group $H_{0}$ as a stabilizer, i.e. $W_{\left(\Gamma, \alpha_{0}\right)}\left(G_{0} / H_{0}\right)$ is isomorphic to the free action of $\hat{G}_{0}=G_{0} / H_{0}$ associated with $\left(Q, u_{0}\right)$, where $u_{0}\left(Q x_{0}, x_{0}\right)=\varphi\left(x_{0}\right)$.

Proof. The proof follows from Theorem 6.4, Lemma 6.1 and Proposition 5.8.

After the preparations made, let us consider the existence and uniqueness theorems (analogous to the theorems of Section 5) for the case, where $\Gamma$ is an ergodic a.f. type II group of automorphisms and $\alpha$ a nonregular cocycle from $Z^{1}(X \times \Gamma, G)$ such that $r(\Gamma, \alpha)=H$.

Let there be defined two pairs $\left(\Gamma_{i}, \alpha_{i}\right), i=1,2$, and let $r\left(\Gamma_{1}, \alpha_{1}\right)=r\left(\Gamma_{2}, \alpha_{2}\right)=$ $H$. According to Theorem 6.4, we can transfer to weakly equivalent pairs which has the following properties $\Gamma_{1}=\Gamma_{2}=\Gamma, \alpha_{1}\left(x_{0}, y, S_{0}\right)=\alpha_{2}\left(x_{0}, y, S_{0}\right)=$ $\beta(y, S)$ (in the case where $\Gamma$ is of type II, $\beta_{0}$ coincides with $\beta$ ).

Theorem 6.6. Let $\Gamma$ be an ergodic a.f. type $I I_{1}$ group of automorphisms of $(X, \mathscr{B}, \mu)$ and $\alpha_{i} \in Z^{1}(X \times \Gamma, G), i=1,2$. Let $W_{\left(\Gamma, \alpha_{1}\right)}(G)$ and $W_{\left(\Gamma, \alpha_{2}\right)}(G)$ be isomorphic and preserve measure; then $r\left(\Gamma, \alpha_{1}\right)=r\left(\Gamma, \alpha_{2}\right)=H$ and the pairs $\left(\Gamma, \alpha_{1}\right)$ and $\left(\Gamma, \alpha_{2}\right)$ are stably weakly equivalent. 
Proof. As has been mentioned, the group $\Gamma$ can be believed to be generated by $\bar{Q}_{0}^{\prime}$ and $S_{0}$ and the cocycles $\alpha_{i}, i=1,2$ to satisfy relations (6.8). The isomorphism of $W_{1}(G)=W_{\left(\Gamma, \alpha_{1}\right)}(G)$ and $W_{2}(G)=W_{\left(\Gamma, \alpha_{2}\right)}(G)$, and Proposition 6.5, and (6.8) lead to the equalities: $Q_{1}=Q_{2}=Q, \varphi_{1}\left(x_{0}\right)=\varphi_{2}\left(x_{0}\right)=\varphi\left(x_{0}\right), \alpha_{1}\left(x_{0}, y, \bar{Q}_{0}^{1}\right)=$ $\alpha_{2}\left(x_{0}, y, \bar{Q}_{0}^{2}\right)$, where $\bar{Q}_{0}^{i}\left(x_{0}, y\right)=\left(Q x_{0}, s_{i}\left(x_{0}\right) U_{i}\left(x_{0}\right) y\right), i=1,2$. Note that $\bar{Q}_{0}^{i}$, $i=1,2$ and $Q$ preserve measure, since also so do $\Gamma$ and $W_{i}(G)$ (see Proposition 6.5). Therefore, we obtain that $\Phi\left(U_{1}\left(x_{0}\right)\right)=\Phi\left(U_{2}\left(x_{0}\right)\right)=1$ for a.a. $x_{0} \in X_{0}$. Thus, we can consider the weakly equivalent pairs for which $U_{1}\left(x_{0}\right)=U_{2}\left(x_{0}\right)=1$. Then, it is sufficient (as in Section 5) to consider the automorphism $Q_{0}=Q \times 1$ instead of $\bar{Q}_{0}^{1}$, defining $\alpha_{1}\left(x_{0}, y, Q_{0}\right)=\bar{\varphi}\left(x_{0}\right)=\sigma\left(\varphi\left(x_{0}\right)\right)$, where $\sigma: G / H \rightarrow G$ was defined in Subsection 6.1. If the statement of the theorem is proved on this assumption, then it will obviously be valid in the general case as well. Thus, the generators of $[\Gamma]$ are chosen in two ways: $\left(Q_{0}, S_{0}\right)$ and $\left(\bar{Q}_{0}, S_{0}\right)$, where $\bar{Q}_{0}=s_{0} Q_{0}$, and $\alpha_{1}$ and $\alpha_{2}$ coincide on $S_{0}$ and are related as follows:

$$
\alpha_{2}\left(x_{0}, y, Q_{0}\right)=\alpha_{1}\left(x_{0}, y, Q_{0}\right)+h\left(x_{0}, y\right)
$$

on $Q_{0}$, where $h\left(x_{0}, y\right)$ is a measurable function with values in $H$.

Let $\left\{\varepsilon_{k}\right\}_{k=1}^{\infty}$ be a sequence of positive numbers monotonically converging to 0 and $\left\{\mathscr{D}_{k}\right\}_{k=1}^{\infty}$ a sequence of sets, which is dense in $\mathscr{B}$, each term occuring an infinite number of times in it. Construct a $\Gamma$-array $\zeta_{1}$ such that the cocycle $\alpha_{1}$ takes constant values on each of its elements. Besides, the group of automorphisms $\mathcal{G}\left(\zeta_{1}\right)$, approximates the orbits of $\Gamma$ with error $\varepsilon_{1}$, and in $\mathcal{Q}\left(\zeta_{1}\right)$ there is a set $D_{1}^{\prime}$ which approximates $D_{1}$ with the error $\varepsilon_{1}$. Such the array exists because the function $\bar{\varphi}\left(x_{0}\right)$ may be thought to be piecewise constant. Since $\alpha_{1}$ on $\left\{Q_{0}^{n}: n \in Z\right\}$ is transient, then the array $\zeta_{1}$ is globally nontransitive and consists of a finite number of transitive components. According to (6.9) and the condition of the theorem, there exists a $\Gamma$-array $\eta_{1}$ such that: (1) $\eta_{1}$ has as many transitive components as $\zeta_{1}$; (2) every transitive compnent of $\eta_{1}$ contains as many sets as the corresponding component of $\zeta_{1}$; (3) the sets of $\eta_{1}$ can be numbered so that for the sets $E(i)$ and $F(i)$ of $\zeta_{1}$ and $\eta_{1}$, respectively, having identical numbers, $\nu\left(E(i)\left(x_{0}\right)\right)=\nu\left(F(i)\left(x_{0}\right)\right)$ for a.a. $x_{0} \in X_{0}$, where $E(i)\left(x_{0}\right)$ and $F(i)\left(x_{0}\right)$ are $x_{0}$ sections of $E(i)$ and $F(i)$; (4) the values of $\alpha_{1}$ and $\alpha_{2}$ on elements of $\zeta_{1}$, and $\eta_{1}$ with identical numbers coincide. E.g., $\eta_{1}$ can be constructed over the partition which defines $\zeta_{1}$ on $X$. Then, refine the $\Gamma$-array $\eta_{1}$ and construct a $\Gamma$-array $\eta_{2}$ such that the cocycle $\alpha_{2}$ takes constant values on its elements and $\mathcal{G}\left(\eta_{2}\right)$ approximates the orbits of $\Gamma$ with the error $\varepsilon_{1}$ and $\mathscr{L}\left(\eta_{2}\right)$ also approximates the set $D_{1}$ with the error $\varepsilon_{1}$. To do so, let us consider a set $A$ consisting of the union 
of fundamental sets of transitive components of $\eta_{1}$ and construct the $\Gamma$-array $\eta_{1}^{\prime}$ on $A$ in such a way that the refinement of $\eta_{1}$ by $\eta_{1}^{\prime}$, which we denote by $\eta_{2}$, should have the above properties. Then, construct a refinement $\zeta_{1}$ of $\zeta_{2}$ so that the above conditions (1)-(4) should be fulfilled for the arrays $\zeta_{2}, \eta_{2}$. By repeating the said procedure a countable number of times, construct two sequence of $\Gamma$-arrays $\left\{\zeta_{k}\right\}_{k=1}^{\infty}$ and $\left\{\eta_{k}\right\}_{k=1}^{\infty}$, which approximate the $\sigma$-algebra $\mathscr{B}$ and the orbits of $\Gamma$. On elements of these arrays having the same numbers, the cocycles $\alpha_{1}$ and $\alpha_{2}$ have the same values. Therefore, as in the proof of Theorem 2.3 of [1] and Theorem 3.9, we conclude that there exists a measure-preserving automorphism $\theta \in N[\Gamma]$ such that $\theta \circ \alpha_{2}=\alpha_{1}$. Note that the automorphism $\theta$ represents a measurable filed of automorphisms $x_{0} \rightarrow \theta\left(x_{0}\right) \in N[S]$. This follows from the fact that for any $k \in \mathbb{N}$ there exists an automorphism $s_{k} \in\left[S_{0}\right]$ such that $\zeta_{k}$ is mapped by $s_{k}$ into $\eta_{k}$ (see property (3) above). Since $\theta$ is the limit in the metric $d$ on $N[\Gamma]$ of the automorphism sequence $\left\{s_{k}\right\}_{k=1}^{\infty}$, then $\theta$ has the above form (the metric $d$ was defined in [9]). Thus, it is proved that the pairs $\left(\Gamma_{1}, \alpha_{1}\right)$ and $\left(\Gamma_{2}, \alpha_{2}\right)$ (under the above assumptions) are stably weakly equivalent.

Consider the problem of existence of a pair $(\Gamma, \alpha)$ for which the associated action is isomorphic to a given action of $G$. We shall consider in particular nonfree actions.

Theorem 6.7. Let an ergodic action $W(G)$ of the group $G$ on a Lebesgue space $(\Omega, p), p(\Omega)=1$ be defined which preserves $p$ and has a stabilizer $H \subset G$. Then there exists a pair $(\Gamma, \alpha)$, where $\Gamma$ is a type $I_{1}$ ergodic a. $f$. group of automorphisms of $(X, \mu)$ and a cocycle $\alpha \in Z^{1}(X \times \Gamma, G)$, such that $r(\Gamma, \alpha)=H$ and the associated action $W_{(\Gamma, \alpha)}(G)$ is isomorphic to $W(G)$.

Proof. The plan of the proof is the same as that of Theorem 5.10. Define for the free action $W(\hat{G})$ of $\hat{G}=G / H$ on $(\Omega, p)$ the following objects as in Thoerem 5.10: $\left(X_{0}, \mu_{0}\right), Q, u_{0},(Y, \nu)$ and $S \in \operatorname{Aut}(Y, \nu)$. Let $\beta \in Z^{1}(Y \times[S], H)$ be the standard cocycle defined in Subsection 3.1 such that $H=r(S, \beta)$. Let $\Gamma$ be a group of automorphisms of $(X, \mu)=\left(X_{0} \times Y, \mu_{0} \times \nu\right)$ generated by $Q_{0}=Q \times \mathbb{1}$ and $S_{0}=\mathbb{1} \times S$. Define a cocycle $\alpha$ for $\Gamma: \alpha\left(x_{0}, y, Q_{0}\right)=\sigma\left(u_{0}\left(Q x_{0}, x_{0}\right)\right)$, $\alpha\left(x_{0}, y, S_{0}\right)=\beta(y, S)$, where $\sigma: \hat{G} \rightarrow G$ is a measurable section of $G$ over $\hat{G}$. As in Theorem 5.10, we see that the pair $(\Gamma, \alpha)$ is sought-for one.

The following theorem shows that the action associated with $(\Gamma, \alpha)$ does not necessarily preserve measure, though $\Gamma$ is a type II group of automorphisms. 
Theorem 6.8. Let $W(G)$ be an ergodic non-free non-singular action of $G$ on a space $(\Omega, p), p(\Omega)=1$ and let $H$ be the stabilizer of the action of $G$. Then, there exists an ergodic a.f. group $\Gamma$ acting on a Lebesgue space $(X, \mu), \mu(X)=\infty$ and preserving the measure $\mu$ and there exists a cocycle $\alpha \in Z^{1}(X \times \Gamma, G)$, and both are such that $r(\Gamma, \alpha)=H$ and $W_{(\Gamma, \alpha)}(G)$ is isomorphic to $W(G)$.

Remark. If $E \subset X, \mu(E)<\infty$, then for the pair $\left(\Gamma_{E}, \alpha_{E}\right)$ the associated action $W_{\left(\Gamma_{E, \infty}, \alpha_{B}\right)}(G)$ is isomorphic to $W_{(\Gamma, \alpha)}(G)$. Therefore, it is unessnetial that $\Gamma$ in Theorem 6.8 is of type $\mathrm{II}_{\infty}$.

Proof. Define the objects $\left(X_{0}, \mu_{0}\right), Q, u_{0}, \sigma, W(\hat{G})$ as in Theorems 5.10 and 6.7. The automorphism $Q$ has a nontrivial Radon-Nikodym cocycle $\rho\left(x_{0}, Q^{n}\right)$ $=\log \frac{d Q^{-n_{\circ}} \mu_{0}}{d \mu_{0}}\left(x_{0}\right)$. Let $S_{1}$ be an ergodic automorphism of a Lebesgue space $\left(Y_{1}, \nu_{1}\right), \nu_{1}\left(Y_{1}\right)=\infty$ preserving the measure $\nu_{1}$. Construct a measurable filed $x_{0} \rightarrow U\left(x_{0}\right) \in N\left[S_{1}\right]\left(x_{0} \in X_{0}\right)$ of automorphisms of $\left(Y_{1}, \nu_{1}\right)$ such that

$$
\Phi\left(U\left(x_{0}\right)\right)=-\log \frac{d Q^{-1} \circ \mu_{0}}{d \mu_{0}}\left(x_{0}\right) .
$$

Let $S,(Y, \nu)$ and $\beta$ be the same as in Thoerem 6.7. Define the automorphism group $F$ generated by commutating automorphisms $1 \times S$ and $S_{1} \times 1$ on the space $\left(Y_{1} \times Y, \nu_{1} \times \nu\right)$ and the cocycle $\beta_{1} \in Z^{1}\left(Y_{1} \times Y \times F, H\right)$, assuming that $\beta\left(y, S^{m}\right)=$ $\beta_{1}\left(y_{1}, y, S_{1}^{n}, S^{m}\right)$. Now it is obvious that $r\left(F, \beta_{1}\right)=H$.

Let us consider the following automorphisms on the space $(X, \mu)=$ $\left(X_{0} \times Y_{1} \times Y, \mu_{0} \times \nu_{1} \times \nu\right)$ :

$$
\begin{aligned}
& Q_{0}\left(x_{0}, y_{1}, y\right)=\left(Q x_{0}, U\left(x_{0}\right) y_{1}, y\right), \\
& S_{1,0}\left(x_{0}, y_{1}, y\right)=\left(x_{0}, S_{1} y_{1}, y\right), \\
& S_{0}\left(x_{0}, y_{1}, y\right)=\left(x_{0}, y_{1}, S y\right) .
\end{aligned}
$$

These automorphisms generate the ergodic a.f. group $\Gamma$, which, by (6.10), is of type $\mathrm{II}_{\infty}$. Define the cocycle $\alpha$ on $\Gamma$ :

$$
\begin{aligned}
& \alpha\left(x_{0}, y_{1}, y, Q_{0}\right)=\sigma\left(u_{0}\left(Q x_{0}, x_{0}\right)\right), \\
& \alpha\left(x_{0}, y_{1}, y, S_{1,0}\right)=0, \\
& \alpha\left(x_{0}, y_{1}, y, S_{0}\right)=\beta(y, S) .
\end{aligned}
$$

It is easy checked that formulae (6.11) define the cocycle $\alpha$ on $\Gamma$ correctly. The ergodicity of $S_{1}$ on $Y$ and of $S(\beta)$ on $Y \times H$ means that the associated action $W_{(\Gamma, \alpha)}(G)$ of $G$ has the stabilizer $H$ and is defined on the quotient space $X_{0} \times G / H$ by the measurable partition into orbits of $\left(x_{0}, \hat{g}\right) \rightarrow\left(Q x_{0}, \hat{g}+u_{0}\left(Q x_{0}, x_{0}\right)\right)$. Since 
$u_{0}$ is a return cocycle for $W(G / H)$, then $W_{(\Gamma, \alpha)}(G)$ is isomorphic to $W(G)$.

Prove now the uniqueness theorem for pairs whose associated actions are non-free and non-singular.

It follows from Theorem 6.4 that, if a weakly equivalent pairs are considered, any pair $(\Gamma, \alpha)$ can be assumed to have the following properties: the group $\Gamma$ is of type II and is generated by the automorphisms $\bar{Q}_{0}:\left(x_{0}, y\right) \rightarrow\left(Q x_{0}, \bar{U}\left(x_{0}\right) y\right)$, $S_{0}:\left(x_{0}, y\right) \rightarrow\left(x_{0}, S y\right)$ acting on $\left(X_{0} \times Y, \mu_{0} \times \nu\right)$, and the cocycle $\alpha$ is given by the formulae:

$$
\begin{aligned}
& \alpha\left(x_{0}, y, \bar{Q}_{0}\right)=\bar{\varphi}\left(x_{0}\right)=\sigma\left(\varphi\left(x_{0}\right)\right), \\
& \alpha\left(x_{0}, y, S_{0}\right)=\beta(y, S),
\end{aligned}
$$

where $\beta$ is the standard cocycle from Section 3 , the function $\varphi: X_{0} \rightarrow \hat{G}$ is outside a neighborhood of identity in $G$ and $\sigma$ is a section of $G$ over $\hat{G}$.

Theorem 6.9. Let there be defined the pairs $\left(\Gamma_{1}, \alpha_{1}\right)$ and $\left(\Gamma_{2}, \alpha_{2}\right)$ satisfying the above conditions and relations (6.12). Then, if the associated actions $W_{\left(\Gamma_{1}, \alpha_{1}\right)}(G)$ and $W_{\left(\Gamma_{2}, \alpha_{2}\right)}(G)$ are isomorphic, then $\left(\Gamma_{1}, \alpha_{1}\right)$ and $\left(\Gamma_{2}, \alpha_{2}\right)$ are stably weakly equivalent, and $r\left(\Gamma_{1}, \alpha_{1}\right)=r\left(\Gamma_{2}, \alpha_{2}\right)$.

Proof. Obviously, it may be believed that $\Gamma_{1}$ and $\Gamma_{2}$ act on the same space $\left(X_{0} \times Y, \mu_{0} \times \nu\right)$ and moreover $\left[\Gamma_{1}\right]=\left[\Gamma_{2}\right]=[\Gamma]$. In view of the results of Section 5 and Proposition 6.8 , by changing to stably weakly equivalent pairs, we can provide that $Q_{1}=Q_{2}=Q, \varphi_{1}\left(x_{0}\right)=\varphi_{2}\left(x_{0}\right)=\varphi\left(x_{0}\right)$. Thus, we are now to construct an automorphism $P=\left(x_{0} \rightarrow P\left(x_{0}\right)\right) \in N[\Gamma]$, where $P\left(x_{0}\right) \in N[S]$ and $P^{-1} \circ \alpha_{1}=\alpha_{2}$, $P^{-1} \bar{Q}_{0}^{1} P=\bar{Q}_{0}^{2} s_{0}$. It follows from the conditions of the theorem and the fact that the automorphisms $\bar{Q}_{0}^{i}, i=1,2$, preserve measure, that for a.a. $x_{0} \in X_{0}$,

$$
\Phi\left(\bar{U}_{1}\left(x_{0}\right)\right)=\Phi\left(\bar{U}_{2}\left(x_{0}\right)\right) .
$$

The values of $\alpha_{1}$ and $\alpha_{2}$ on $\bar{Q}_{0}^{1}$ and $\bar{Q}_{0}^{2}$ differ by a function taking values in the group $H=r(S, \beta)$. We may multiply the element $\bar{Q}_{0}^{2}$ by an automorphism $s_{0}^{\prime} \in\left[S_{0}\right]$, so that the values of $\alpha_{1}$ and $\alpha_{2}$ on $\bar{Q}_{0}^{1}$ and $\bar{Q}_{0}^{2} s_{0}^{\prime}$ should become equal.

Now we shall do as in the proof of Theorem 6.6. Using the above properties of $\alpha_{1}$ and $\alpha_{2}$ and the property (6.13), construct two sets of $\Gamma$-arrays, $\left\{\xi_{n}\right\}_{n=1}^{\infty}$ and $\left\{\eta_{n}\right\}_{n=1}^{\infty}$ satisfying the conditions: (1) $\cup_{n=1}^{\infty} \mathcal{G}\left(\xi_{n}\right) x=\cup_{n=1}^{\infty} \mathcal{G}\left(\eta_{n}\right) x$ $=\Gamma x$; (2) $\sigma\left(\cup_{n=1}^{\infty} \mathscr{L}\left(\xi_{n}\right)\right)=\sigma\left(\cup_{n=1}^{\infty} \mathscr{L}\left(\eta_{n}\right)\right)=\mathscr{B}$; (3) the arrays $\xi_{n}$ and $\eta_{n}, n \in \mathbb{N}$ have an equal number of sets and $\nu\left(E(i)\left(x_{0}\right)\right)=\nu\left(F(i)\left(x_{0}\right)\right)$ for a.a. $x_{0} \in X_{0}$, where 
$E(i)$ and $F(i)$ are sets from $\xi_{n}$ and $\eta_{n}$; (4) on elements of $\xi_{n}$ and $\eta_{n}$ having the same serial numbers the cocycles $\alpha_{1}$ and $\alpha_{2}$ assume equal values. Therefore, there exists an automorphism $P=\left(x_{0} \rightarrow P\left(x_{0}\right)\right) \in N[\Gamma]$, which transforms the generators $\left(\bar{Q}_{0}^{1}, S_{0}\right)$ of $\Gamma$ into $\left(\bar{Q}_{0}^{2}, S_{0}\right)$ and is such that $P^{-1} \circ \alpha_{1}=\alpha_{2}$.

\section{§7. Associated Actions for Type III Groups $\Gamma$}

7.1. In this section we shall consider the existence and uniqueness theorems for the pairs $\left(\Gamma, \alpha_{0}\right)$, where $\Gamma$ is a type III a.f. ergodic group of automorphisms. In Subsection 7.2 we shall construct an example of $\left(\Gamma, \alpha_{0}\right)$ for which $r(\Gamma, \alpha)=G, r(\Gamma, \rho)=\boldsymbol{R}$ (i.e. $\Gamma$ is of type $\mathrm{III}_{1}$ ), but $r\left(\Gamma, \alpha_{0}\right)=\{0\}$. In Subsection 7.4 we shall study the interrelation between the type of $\Gamma$ and that of an associated action.

We shall use essentailly the results of the two preceding sections. First, we shall consider free associated actions of $G_{0}=G \times \boldsymbol{R}$, where $G$ is an arbitrary 1.c.s. abelian group. Recall that we deal here with nonregular recurrent cocycle $\alpha_{0}$.

It follows from Theorem 5.6 that, if $\left(\Gamma, \alpha_{0}\right)$ is such that $W_{\left(\Gamma, \alpha_{0}\right)}\left(G_{0}\right)$ is free, i.e. $r\left(\Gamma, \alpha_{0}\right)=\{0\}$, then $\Gamma$ acts on the space $\left(X_{0} \times Y, \mu_{0} \times \nu\right)$, and $\left(\Gamma, \alpha_{0}\right)$ has the following properties: (1) $\Gamma$ is generated by the automorphisms $Q_{0}$ and $S_{0}$ (see (6.1)); (2) $\alpha_{0}$ is defined on $Q_{0}$ and $S_{0}$ by the formulae: $\alpha_{0}\left(x_{0}, y, Q_{0}\right)=\varphi\left(x_{0}\right)$, $\alpha_{0}\left(x_{0}, y, S_{0}\right)=0$, where the function $\varphi\left(x_{0}\right)$ is separated from the identity in $G_{0}$ by a neighborhood $V_{0}$.

Property (2) implies that $\alpha_{0}$, when considered on $\left\{Q_{0}^{n}: n \in Z\right\}$, is there transient, and the ergodic automorphism $S$ is of type II, i.e. $S \circ \nu=\nu$. Assume for convenience the measure $\nu$ to be infinite. Besides, since $\varphi\left(x_{0}\right)=\left(\varphi_{G}\left(x_{0}\right), \varphi_{R}\left(x_{0}\right)\right)$, then

$$
\varphi_{R}\left(x_{0}\right)=\log \frac{d Q^{-1} \circ \mu_{0}}{d \mu_{0}}\left(x_{0}\right)+\Phi\left(U\left(x_{0}\right)\right)
$$

The above properties of $\left(\Gamma, \alpha_{0}\right)$ suggest validity of the following lemma (which is proved in the same way as Lemma 5.8).

Lemma 7.1. The free action $W_{\left(\Gamma, \alpha_{0}\right)}\left(G_{0}\right)$ of $G$ associated with $\left(\Gamma, \alpha_{0}\right)$ is isomorphic to the associated action of $G_{0}$ constructed by the pair $\left(Q, \varphi\left(x_{0}\right)\right)$.

Theorem 7.2. Let there be two pairs $\left(\Gamma_{1}, \alpha_{0}^{1}\right)$ and $\left(\Gamma_{2}, \alpha_{0}^{2}\right)$, which satisfy the above conditions and are such that $r\left(\Gamma_{1}, \alpha_{0}^{1}\right)=r\left(\Gamma_{2}, \alpha_{0}^{2}\right)=\{0\}$. Then, the isomorphism of the associated actions $W_{\left(\Gamma_{1}, \alpha_{0}^{2}\right)}\left(G_{0}\right)$ and $W_{\left(\Gamma_{2} \alpha_{0}^{2}\right)}\left(G_{0}\right)$ means that $\left(\Gamma_{1}, \alpha_{0}^{1}\right)$ 
and $\left(\Gamma_{2}, \alpha_{0}^{2}\right)$ are weakly equivalent.

The proof is essentially the same as that of Theorem 5.9.

Theorem 7.3. Let $W\left(G_{0}\right)$ be a free ergodic action of $G_{0}$ on a Lebesgue space $(\Omega, p)$. Then, there exists a pair $\left(\Gamma, \alpha_{0}\right)$, where $\Gamma$ is an ergodic a.f. group of automorphisms of $(X, \mathscr{B}, \mu)$ and $\alpha_{0} \in Z^{1}\left(X \times \Gamma, G_{0}\right)$ both such that the associated action $W_{\left(r_{,} \alpha_{0}\right)}\left(G_{0}\right)$ is isomorphic to $W\left(G_{0}\right)$.

Proof. We introduce, as in Theorem 5.11, the following objects: $\left(X_{0}, \mu_{0}\right)$, $Q, u_{0},(Y, \nu), S . \quad$ Denote

$$
u_{0}\left(Q x_{0}, x_{0}\right)=\varphi\left(x_{0}\right)=\left(\varphi_{G}\left(x_{0}\right), \varphi_{R}\left(x_{0}\right)\right) .
$$

Choose a measurable field of automorphisms $x_{0} \rightarrow U\left(x_{0}\right) \in N[S]$ such that

$$
\Phi\left(U\left(x_{0}\right)\right)=\varphi_{R}\left(x_{0}\right)-\log \frac{d Q^{-1} \circ \mu_{0}}{d \mu_{0}}\left(x_{0}\right) .
$$

Let $\Gamma$ be the group of automorphisms with the generators $Q_{0}$ and $S_{0}$, where $Q_{0}$ and $S_{0}$ are defined by (6.1). The cocycle $\alpha$ is defined by the formulae: $\alpha\left(x_{0}, y, Q_{0}\right)=\varphi_{G}\left(x_{0}\right), \alpha\left(x_{0}, y, S_{0}\right)=0$. The action $W_{\left(\Gamma, \alpha_{0}\right)}\left(G_{0}\right)$ is isomorphic to $W(G)$.

In Subsection 7.4 we shall consider the problem of the type of the group $\Gamma$ whose existence was proved in Theorem 7.3.

7.2. Let us construct an example of a type $\mathbb{I I I}_{1}$ automorphism group $\Gamma$ and a cocycle $\alpha \in Z^{1}(X \times \Gamma, G)$, which are such that the ranges of the components $\alpha$ and $\rho$ of $\alpha_{0}$ are $G$ and $\mathbb{R}$, respectively, while $\alpha_{0}=(\alpha, \rho)$ is lacunary in $G_{0}$.

Example 7.4. Let there be defined a Lebesgue space $(X, \mu)=\left(X_{0} \times Y\right.$, $\left.\mu_{0} \times \nu\right), \mu_{0}\left(X_{0}\right)=\nu(Y)=\infty$, an ergodic automorphism $Q \in \operatorname{Aut}\left(X_{0}, \mu_{0}\right), Q \circ \mu_{0}=\mu_{0}$, an ergodic automorphism $S \in \operatorname{Aut}(Y, \nu), S \circ \nu=\nu$, and an automorphism $U \in$ Aut $(Y, \nu)$, the latter such that $U \in N[S]$ and $U \circ \nu=\lambda \nu$, where $\lambda \in(0,1)$. Put as usual $Q_{0}\left(x_{0}, y\right)=\left(Q x_{0}, U y\right), S_{0}\left(x_{0}, y\right)=\left(x_{0}, S y\right)$. Since $Q$ is of type $\mathbb{I}_{\infty}$, then there exists an action $l(G)$ of $G$ on $\left(X_{0}, \mu_{0}\right)$, which preserves the measure $\mu_{0}$ and is such that $l(g) \in N[Q], g \in G$ and $l(g) \notin[Q], g \neq e$ (see e.g. [6]). Put $l_{0}(g)\left(x_{0}, y\right)$ $=\left(l(g) x_{0}, U(g) y\right), g \in G$, where $U(g) \in N[S]$ and $\Phi(U(g))=\log \lambda(g)$, the numbers $\log \lambda, \log \lambda(g), g \in G$ being rationally independent, and assume that there exists an element $g_{1} \in G$ such that $\lambda\left(g_{1}\right) \neq 1$. Consider equivalence relation $\mathcal{E}$ with continuous orbits, which is generated on $(X, \mu)$ by the action of $Q_{0}, S_{0}$ and $l_{0}(g)=l(g) \times U(g), g \in G$. The above properties of $\lambda(g)$ show that the equiva- 
lence relation $\varepsilon$ is of type III $_{1}$. Consider the cocycle $\alpha$ deffned on the generators of $\mathcal{E}$ as follows: $\alpha\left(x_{0}, y, Q_{0}\right)=\alpha\left(x_{0}, y, S\right)=0, \alpha\left(x_{0}, y, l_{0}(g)\right)=g, g \in G$. Obviously, $r(\mathcal{E}, \alpha)=G$. Calculate now the range of $\alpha_{0}=(\alpha, \rho)$. Show that the associated action $W_{\left(\mathcal{E}, \alpha_{0}\right)}\left(G_{0}\right)$ is free, whence it will follow that $r\left(\mathcal{E}, \alpha_{0}\right)=\{0\}$. The orbits of the equivalence relation $\mathcal{E}\left(\alpha_{0}\right)$ are generated by the automorphisms:

$$
\begin{aligned}
& Q_{0}\left(\alpha_{0}\right)\left(x_{0}, y, h, u\right)=\left(Q_{0}\left(x_{0}, y\right), h, u+\log \lambda\right), \\
& S_{0}\left(\alpha_{0}\right)\left(x_{0}, y, h, u\right)=\left(x_{0}, S y, h, u\right), \\
& l_{0}(g)\left(\alpha_{0}\right)\left(x_{0}, y, h, u\right)=\left(l(g) x_{0}, U(g) y, h+g, u+\log \lambda(g)\right)
\end{aligned}
$$

(recall that $\varepsilon\left(\alpha_{0}\right)$ defines the partition into equivalence classes on the space $\left.X_{0} \times Y \times G \times \boldsymbol{R}\right)$. Let us find now the quotient space by the measurable hall of partition into orbits of the equivalence relation $\mathcal{E}\left(\alpha_{0}\right)$ on $X_{0} \times Y \times G \times \mathbb{R}$. Since $S$ acts ergodically on $Y$ and in view of (7.2), this quotient space should be sought for in the set $X_{0} \times G \times \mathbb{R}$, i.e. in "the plane perpendicular to $Y$ ". Put $E=X_{0} \times\{0\} \times[0,-\log \lambda)$. It follows from (7.2) that the set $E$ intersects the orbits of $Q_{0}\left(\alpha_{0}\right)$ and $l_{0}(g)\left(\alpha_{0}\right)$ exactly at one point. Therefore, $E$ can be identified with the desired quotient space, and $W_{\left(\mathcal{E}, \alpha_{0}\right)}\left(G_{0}\right)$ can be thought to be defined on $E$. Let $\left(g_{0}, u_{0}\right) \in G_{0},\left(x_{0}, 0, u\right) \in E$; then

$$
W_{\left(\mathcal{E}, \alpha_{0}\right)}\left(g_{0}, u_{0}\right)\left(x_{0}, 0, u\right)=Q_{0}^{n}\left(\alpha_{0}\right)\left(l\left(-g_{0}\right) x_{0}, 0, u+u_{0}-\log \lambda\left(g_{0}\right)\right),
$$

where the number $n$ is chosen by the condition $u+u_{0}-\log \lambda\left(g_{0}\right)+n \log \lambda \in$ $[0,-\log \lambda]$. Therefore, the automorphism $W_{\left(\mathcal{E}, \alpha_{0}\right)}\left(g_{0}, u_{0}\right)$ acts identically if and only if $Q^{n} l\left(-g_{0}\right) x_{0}=x_{0}$. However, since $l(G)$ was chosen to be strictly outer to $[Q]$, the latter equality is not true.

Let us construct the countable group $\Gamma$ of type $\mathrm{III}_{1}$ in the following way. Let $\mathcal{G}_{1}$ be the measurable groupoid generated by [Q] and $l(g), g \in G$. Then $\mathcal{G}_{1}$ is isomorphic to the groupoid $X^{\prime} \times \mathbb{T} \times \Gamma \times T$, where a countable automorphism group $\Gamma$ acts on the Lebesgue space $X^{\prime}[3,17]$. The cocycle $\alpha$ defined on $\mathcal{G}_{1}$ can be replaced by the cohomologous cocycle $\alpha^{\prime}$ so that it should become trivial for the action of the circle $T$ on itself, i.e. $\alpha^{\prime}$ should be concentrated on $X^{\prime} \times \Gamma$ (simple arguments omitted). The pair $\left(\Gamma, \alpha^{\prime}\right)$ will have the properties: $r\left(\Gamma, \alpha^{\prime}\right)$ $=G, r\left(\Gamma, \alpha_{0}^{\prime}\right)=\{0\}$.

The latter example suggests that regularity of $\alpha$ does not imply regularity of $\alpha_{0}$.

7.3. Consider the uniqueness and existence theorems for $\left(\Gamma, \alpha_{0}\right)$ for the 
case of type III group $\Gamma$ and a non-free associated action $W_{\left(\Gamma, \alpha_{0}\right)}\left(G_{0}\right)$.

Recall the results of Theorem 6.4. By changing to the weakly equivalent pair, one can provide the following properties of any $\left(\Gamma, \alpha_{0}\right)$ such that $r\left(\Gamma, \alpha_{0}\right)=$ $H_{0}$ : (1) the group $\Gamma$ is generated by $Q_{0}$ and $S_{0}$ acting on $\left(X_{0} \times Y, \mu_{0} \times \nu\right)$ as $Q_{0}\left(x_{0}, y\right)=\left(Q x_{0}, U\left(x_{0}\right) y\right), S_{0}\left(x_{0}, y\right)=\left(x_{0}, S y\right), U\left(x_{0}\right) \in N[S],(2) \alpha_{0}$ is defined on $\Gamma$ as

$$
\begin{aligned}
& \alpha_{0}\left(x_{0}, y, Q_{0}\right)=\varphi\left(x_{0}\right)=\left(\varphi_{G}\left(x_{0}\right), \varphi_{R}\left(x_{0}\right)\right), \\
& \alpha_{0}\left(x_{0}, y, S_{0}\right)=\beta_{0}(y, S),
\end{aligned}
$$

where $\beta_{0}$ is the standard cocycle from Section 3, for which $r\left(S, \beta_{0}\right)=H_{0}$. For $S$ there are two alternatives: it is either of type II or of type $\mathrm{III}_{\lambda}(0<\lambda \leq 1)$, according to the form of $H_{0} \subset G \times \mathbb{R}$.

Theorem 7.5. Let the pairs $\left(\Gamma_{i}, \alpha_{0}^{i}\right), i=1,2$ have the above properties and $r\left(\Gamma_{1}, \alpha_{0}^{1}\right)=r\left(\Gamma_{2}, \alpha_{0}^{2}\right)=H_{0}$. Then, if the associated actions $W_{\left(\Gamma_{1}, \alpha_{0}^{1}\right)}\left(G_{0}\right)$ and $W_{\left(\Gamma_{2}, \alpha_{0}^{2}\right)}$ $\left(G_{0}\right)$ are isomorphic, then $\left(\Gamma_{1}, \alpha_{0}^{1}\right)$ and $\left(\Gamma_{2}, \alpha_{0}^{2}\right)$ are weakly equivalent.

Proof. It follows from the conditions of the theorem and relations (7.3) that for both the pairs $\left(\Gamma_{1}, \alpha_{0}^{1}\right)$ and $\left(\Gamma_{2}, \alpha_{0}^{2}\right)$ it can be believed that the Lebesgue spaces coincide in which the groups $\Gamma_{1}$ and $\Gamma_{2}$ act, and also $S_{0}^{1}=S_{0}^{2}$. Since $S$ is of type II or III $_{\lambda}(0<\lambda \leq 1)$, then there exists in $[S]$ an ergodic subgroup on which the cocycle $\beta_{0}$ is trivial (see Corollary 3.15). Thus, as in Lemma 6.5, the associated action $W_{\left(\Gamma_{i}, \alpha_{2}^{i}\right)}\left(G_{0}\right)$ is isomorphic to the action of $G_{0}$ on the quotient space of $X_{0} \times \hat{G}_{0}$ by the measurable partition into orbits of $\left(x_{0}, \hat{g}_{0}\right) \rightarrow\left(Q^{i} x_{0}, \hat{g}+\hat{\varphi}^{i}\right.$ $\left.\left(x_{0}\right)\right), i=1,2$, where $\hat{\varphi}^{i}\left(x_{0}\right)$ is separated from zero. By changing mentally to weakly equivalent pairs, we find that $Q^{1}=Q^{2}=Q$ and $\hat{\varphi}^{1}=\hat{\varphi}^{2}=\varphi$. The latter equality, combined with (7.3), means that $\varphi^{1}$ and $\varphi^{2}$ differ by an element of $H_{0}$, and thus,

$$
\varphi_{G}^{1}\left(x_{0}\right)=\varphi_{G}^{2}\left(x_{0}\right)=h_{G}\left(x_{0}\right), \quad \varphi_{R}^{1}\left(x_{0}\right)=\varphi_{R}^{2}\left(x_{0}\right)=h_{R}\left(x_{0}\right) .
$$

Since $\varphi_{R}^{i}\left(x_{0}\right)=\rho\left(x_{0}, Q\right)+\Phi\left(U^{i}\left(x_{0}\right)\right), i=1,2$, then (7.4) leads to

$$
\Phi\left(U^{1}\left(x_{0}\right)\right)=\Phi\left(U^{2}\left(x_{0}\right)\right)+h_{R}\left(x_{0}\right) .
$$

Relation (7.5) means that by multiplying $Q_{0}^{2}$ by an element $s_{0} \in\left[S_{0}\right]$ (as in the proof of Theorem 6.4), we shall obtain that $\alpha_{0}^{1}$ on $Q_{0}^{1}$ and $\alpha_{0}^{2}$ on $\bar{Q}_{0}^{2}=s_{0} Q_{0}^{2}$ coincide. Now, in view of Theorems 6.6 and 6.9, there exists a transformation $P=\left(x_{0} \rightarrow P\left(x_{0}\right)\right)$ with the following properties: $P \in N\left[S_{0}\right], \Phi\left(P\left(x_{0}\right)\right)=1, P Q_{0}^{1} P^{-1}$ $=s_{0}^{\prime} Q_{0}^{2}, P^{-1} \circ \alpha_{0}^{1}=\alpha_{0}^{2}$. By repeating the arguments of Theorem 6.9, we find that 
$\left(\Gamma_{1}, \alpha_{0}^{1}\right)$ and $\left(\Gamma_{2}, \alpha_{0}^{2}\right)$ are weakly equivalent.

The proof of Theorem 7.5 shows that its conditions provide for orbital equivalence of $\Gamma_{1}$ and $\Gamma_{2}$.

Theorem 7.6. Let $W\left(G_{0}\right)$ be an ergodic non-free action of $G_{0}$ on a Lebesgue space $(\Omega, p)$, for which $p$ is non-singular, and $H_{0}$ the stabilizer of $W\left(G_{0}\right)$. Then, there exist an ergodic a.f. group $\Gamma$ acting on $(X, \mu)$ and a cocycle $\alpha_{0} \in$ $Z^{1}\left(X \times \Gamma, G_{0}\right)$, and both are such that $r\left(\Gamma, \alpha_{0}\right)=H_{0}$ and the associated action $W_{\left(r, \alpha_{0}\right)}\left(G_{0}\right)$ is isomorphic to $W\left(G_{0}\right)$.

Proof. This one is partly similar to those of Theorems 6.8 and 7.3.

7.4. In conclusion, let us consider several results on the relationship between the types of $\Gamma$ and $W_{\left(\Gamma, \alpha_{0}\right)}\left(G_{0}\right)$. Let the group $\Gamma$ be generated, as before, by $Q_{0}$ and $S_{0}$ and the cocycle $\alpha_{0}$ satisfy relations (7.3).

Proposition 7.7. Let $\Gamma$ be an arbitrary countable ergodic group of automorphisms of $(X, \mathcal{B}, \mu)$ and $\alpha$ a transient cocycle from $Z^{1}(X \times \Gamma, G)$, where $G$ is an arbitrary l.c.s. group. The type of $\Gamma$ coincides with that of the associated action $W_{(\Gamma, \alpha)}(G)$ (and also with that of $W_{\left(\Gamma, \alpha_{0}\right)}\left(G_{0}\right)$ ).

The proof follows from the results of Section 2.

Corollary 7.8. The type of the associated action $W_{\left(\Gamma, \alpha_{0}\right)}\left(G_{0}\right)$ for the group $\Gamma$ generated by $Q_{0}$ and $S_{0}$ and for the cocycle $\alpha_{0}$ having the properties mentioned in the beginning of this section coincides with the type of the automorphism $Q$.

Proof. The statement follows from Proposition 6.5.

Consider the following problem: let an action $W\left(G_{0}\right)$ be defined on a Lebesgue space $(\Omega, p)$; then, what type may $\Gamma$ be of, if $W_{\left(\Gamma, \alpha_{0}\right)}\left(G_{0}\right)$ is isomorphic to $W\left(G_{0}\right)$ ? Let us first dwell on some particular cases.

Lemma 7.9. For any pair $\left(\Gamma, \alpha_{0}\right)$, we have $r\left(\Gamma, \alpha_{0}\right) \subset r(\Gamma, \alpha) \times r(\Gamma, \rho)$.

Proposition 7.10. Let the pair $\left(\Gamma, \alpha_{0}\right)$ be such that $\Gamma$ is of type $I I$ or $I I I_{0}$ and the associated action $W_{\left(\Gamma, \alpha_{0}\right)}\left(G_{0}\right)$ is non-free, i.e. $r\left(\Gamma, \alpha_{0}\right)=H_{0} \neq\{0\}$. Then, the group $H_{0}$ is $H_{G} \times\{0\}$ where $H_{G}$ is a closed subgroup of $G$.

The proof immediately follows from Lemma 7.9.

Theorem 7.11. Let $\Gamma$ be an arbitrary ergodic a.f. group of automorphisms of $(X, \mathscr{B}, \mu)$ and $G$ an arbitrary l.c.s. abelian group. There exists a cocycle 
$\alpha_{0} \in Z^{1}(X \times \Gamma, G)$ such that the associated action $W_{\left(\Gamma, \alpha_{0}\right)}\left(G_{0}\right)$ is of an arbitrary type, i.e. the type of $W_{\left(\Gamma, \alpha_{0}\right)}\left(G_{0}\right)$ does not depend on the type of $\Gamma$.

Proof. (1) Let $\Gamma$ be of type II. It can be thought of as generated by $Q_{0}$ and $S_{0}$ preserving measure on $\left(X_{0} \times Y, \mu_{0} \times \nu\right)$ where $Q_{0}\left(x_{0}, y\right)=\left(Q x_{0}, U\left(x_{0}\right) y\right)$, $S_{0}\left(x_{0}, y\right)=\left(x_{0}, S y\right), U\left(x_{0}\right) \in N[S], \Phi\left(U\left(x_{0}\right)\right)=-\rho\left(x_{0}, Q\right)$. Let the cocycle $\alpha_{0}$ be a transient on $Q_{0}$ and be zero on $S_{0}$. Then, the statement of the theorem follows from Corollary 7.8 and Theorem 5.11, because $Q$ can be of an arbitrary type.

(2) Let $\Gamma$ be of type $\mathbb{I I}_{\lambda}(0<\lambda<1)$ or $\mathbb{I I}_{1}$. Then $\Gamma$ is orbitally equivalent to $\Gamma \times \Gamma_{1}$, where $\Gamma_{1}$ is of type II group. Let a cocycle $\alpha \in Z^{1}\left(X \times \Gamma \times \Gamma_{1}\right.$, G) be zero on $\Gamma$ and be concentrated on $\Gamma_{1}$. Then the associated action $W_{\left(\Gamma \times \Gamma_{1}, \alpha_{0}\right)}\left(G_{0}\right)$ is isomorphic to $W_{(\Gamma, \rho)}(\mathbb{R}) \times W_{\left(\Gamma_{1}, \alpha\right)}(G)$. For the cases under consideration, $W_{(\Gamma, \rho)}(\mathbb{R})$ is either a transitive, or a trivial flow. Thus, the type of $W_{\left(\Gamma \times \Gamma_{1}, \alpha_{0}\right)}\left(G_{0}\right)$ is determined by that of $W_{(\Gamma, \alpha)}(G)$ which, by (1), can be arbitrary.

(3) Let $\Gamma$ be of type $\mathbb{I I I}_{0}$. It can be shown, by the same method as in (2), that if $W_{(\Gamma, \rho)}(\mathbb{R})$ preserves measure, then $W_{\left(\Gamma, \alpha_{0}\right)}\left(G_{0}\right)$ may be of an arbitrary type. Consider the case where $W_{(\Gamma, \rho)}(\boldsymbol{R})$ is non-singular. Use the Krieger representation of a type $\mathbb{I I I}_{0}$ group $\Gamma$ as a group $\mathcal{G}(Q, \varphi)$ generated on $\left(X_{0} \times Y, \mu_{0} \times \nu\right), \nu(Y)=\infty$ by the automorphisms $Q_{0}\left(x_{0}, y\right)=\left(Q x_{0}, U\left(x_{0}\right) y\right), S_{0}$ $\left(x_{0}, y\right)=\left(x_{0}, S y\right)$ such that $\rho\left(x_{0}, y, Q_{0}\right)=\rho\left(x_{0}, Q\right)+\Phi\left(U\left(x_{0}\right)\right)=\varphi\left(x_{0}\right)>C>0$ and $\rho\left(x_{0}, y, S_{0}\right)=0[10]$. The flow $W_{(r, \rho)}(\mathbb{R})$ is a special flow constructed from the basis automorphism $Q$ and the ceiling function $\varphi\left(x_{0}\right)$. Replace $\mathcal{G}(Q, \varphi)$ by an orbitally equivalent group. Consider on $\left(X_{0} \times Y \times Y_{1}, \mu_{0} \times \nu \times \nu_{1}\right)$ an automorphism group $\Gamma^{\prime}$ whose generators act as follows

$$
\begin{aligned}
& Q_{0}^{\prime}\left(x_{0}, y, y_{1}\right)=\left(Q x_{0}, U_{2}\left(x_{0}\right) y, U_{1}\left(x_{0}\right) y_{1}\right), \\
& S_{0}^{\prime}\left(x_{0}, y, y_{1}\right)=\left(x_{0}, S y, y_{1}\right), \\
& S_{0}^{\prime \prime}\left(x_{0}, y, y_{0}\right)=\left(x_{0}, y, S_{1} y_{1}\right),
\end{aligned}
$$

where $S_{1} \circ \nu_{1}=\nu_{1}, U_{1}\left(x_{0}\right) \in N\left[S_{1}\right], U_{2}\left(x_{0}\right) \in N[S], \Phi\left(U_{2}\left(x_{0}\right)\right)=\varphi\left(x_{0}\right), \Phi\left(U_{1}\left(x_{0}\right)\right)=$ $-\rho\left(x_{0}, Q\right)$. It is easy to calculate that $W_{\left(\Gamma^{\prime}, \rho\right)}(\mathbb{R})=W_{(\Gamma, \rho)}(\mathbb{R})$. Define a cocycle $\alpha \in Z^{1}\left(X_{0} \times Y \times Y_{1} \times \Gamma^{\prime}, G\right)$ assuming $\alpha$ to be zero on $Q_{0}^{\prime}$ and $S_{0}^{\prime}$ to be equal to $g$ on $S_{0}^{\prime \prime}$ where $g \in G\left(G\right.$ is assumed non-compact). Calculate $W_{\left(\Gamma^{\prime}, \alpha_{0}\right)}\left(G_{0}\right)$. The cocycle $\alpha_{0}$ is zero on $S_{0}^{\prime}$. Let $\left(X_{0} \times Y, \mu_{0} \times \nu_{1}\right)=\left(X_{0}^{\prime}, \mu_{0}^{\prime}\right)$ and the full automorphism group $\left[Q^{\prime}\right]$ of $X_{0}^{\prime}$ be generated by $\left(x_{0}, y_{1}\right) \rightarrow\left(Q x_{0}, U_{1}\left(x_{0}\right) y\right)$ and $\left(x_{0}, y_{1}\right) \rightarrow$ $\left(x_{0}, S_{1} y_{1}\right)$. The automorphism $Q^{\prime}$ obviously preserves the measure $\mu_{0}^{\prime}$. Since $U_{1}\left(x_{0}\right) \in N\left[S_{1}\right]$ and $\Phi\left(U_{1}\left(x_{0}\right)\right) \neq 1$ for a.a. $x_{0} \in X_{0}$, the cocycle $\alpha_{0}=(\alpha, \rho)$ is transient 
on $\bar{Q}_{0}^{\prime}:\left(x_{0}^{\prime}, y\right) \rightarrow\left(Q^{\prime} x_{0}^{\prime}, V\left(x_{0}\right) y\right)$, where $V\left(x_{0}\right) \in N[S]$ and is constructed from $U_{2}\left(x_{0}\right)$. Because $Q^{\prime}$ preserves measure, then $W_{\left(\Gamma^{\prime}, \alpha_{0}\right)}\left(G_{0}\right)$ will also be of type II (see (7.8)).

(4) By combining the methods of (2) and (3), we can provide that the action $W_{\left(\Gamma, \alpha_{0}\right)}\left(G_{0}\right)$ (for $\Gamma$ of type $\left.\operatorname{III}_{0}\right)$ should have type $\operatorname{III}_{\lambda}(0 \leq \lambda \leq 1)$.

\section{References}

[1] Bezuglyi, S.I. and Golodets, V.Ya., Outer conjugacy for aciions of countable amenable groups on measure space, (in Russian), Izv. AN SSSR, Ser. Mat., 50 (1986), 604-621.

[2] Fedorov, A.L., The Krieger theorem for cocycles, (in Russian) Dep. at VINITI 25.02.85, No. 1406-85 Dep.

[3] Feldman, J., Hahn P. and Moore, C.C., Orbit structure and countable sections for actions of continuous group, Adv. Math., 28 (1978), 186-230.

[4] Feldman, J. and Moore, C.C., Ergodic equivalence relations, cohomology, and von Neumann algebras, I, Trans. Amer. Math. Soc., 234 (1977), 289-324.

[5] Golodets, V.Ya. and Sinelshchikov, S.D., Existence and uniqueness of cocycles of an ergodic automorphism with dense images in amenable groups, (in Russian), Kharkov, 1983 (Preprint of FTINT AN USSR, 19-83).

[6] - Outer conjugacy for actions of continuous amenable groups, Publ. RIMS, Kyoto Univ., 23 (1987), 737-769.

[7] - Structure of automorphisms of measurable groupoids and comparison of transient cocycles, (in Russian), Dokl. AN USSR, ser. A, (1987), No. 5, 3-5.

[8] Kornfeld, I.P., Sinay, Ya. G. and Fomin, S.V., Ergodic theory, (in Russian), Nauka, Moscow, 1980.

[9] Hamachi, T. and Osikawa, M., Ergodic groups of automorphisms and Krieger's theorems, Sem. Math. Scl. Keio Univ., (1981), No. 3, 1-113,

[10] Krieger, W., On ergodic flows and isomorphisms of factors, Math. Ann., 223 (1976), 19-70.

[11] Mackey, G.W., Virtual groups and group actions, Math. Ann. 166 (1966), 187-207.

[12] Ramsay, A., Virtual groups and group actions, Adv. Math., 6 (1971), 253-322.

[13] — Topologies on measured groupoids, Univ. of Colorado, 1983.

[14] Rokhlin, V.A., Selected problems of the m.trical theory of dynamical systems, (in Russian), Uspekhi Mat. Nauk, 4 (1949), 57-128.

[15] Schmidt, K., Lecture on cocycles of ergudic transformation groups, Univ. of Warwick, 1976.

[16] Stepin, A.M., Cohomologies of groups of automorphisms of the Lebesgue space, Funkts. Anal. i Prilozh., (in Russian) 5 (1971), 91-92. 
